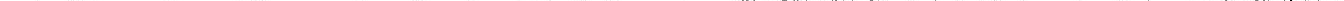




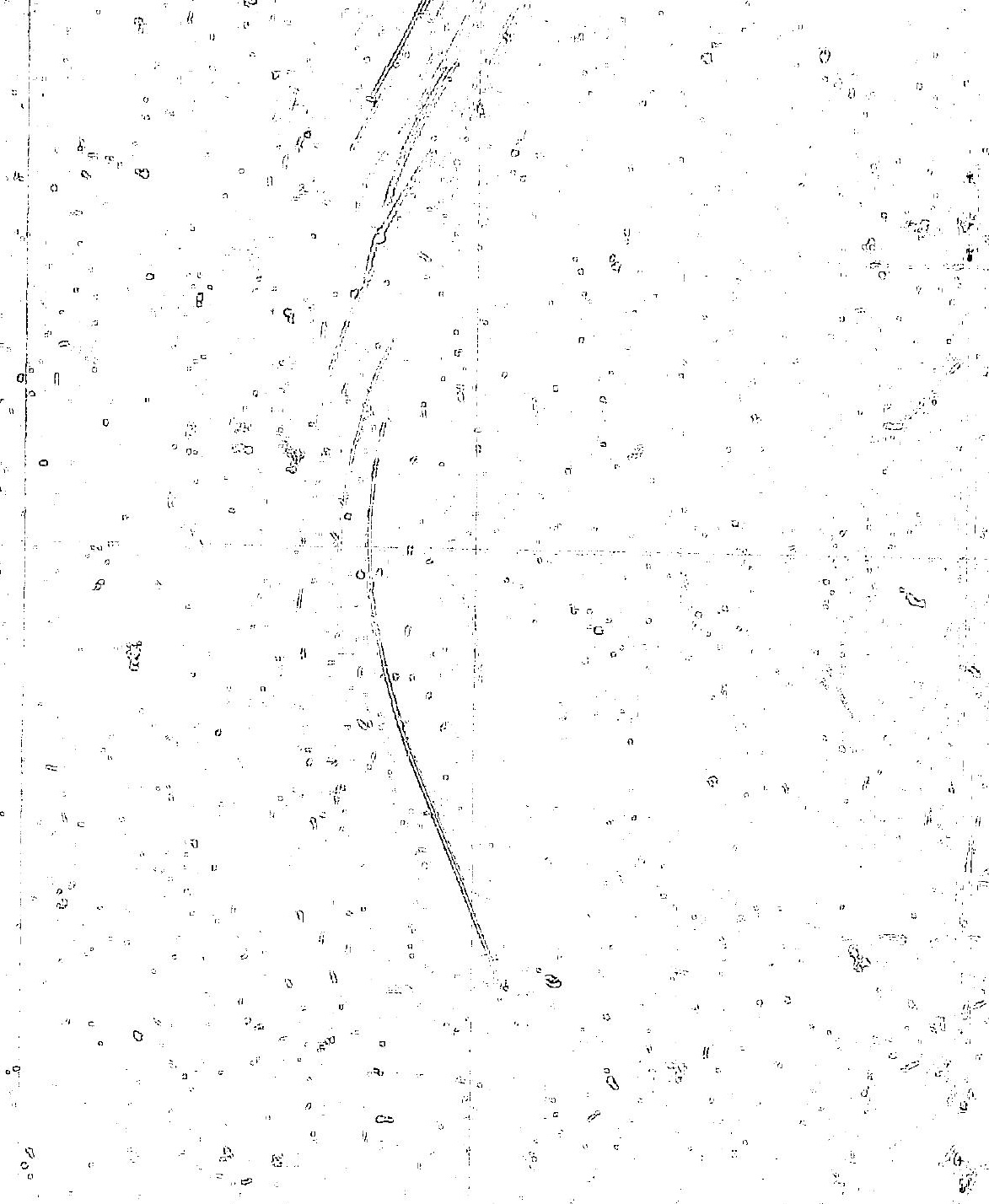

Frinted in the United States of America Available from

National Technical Iriformation Strice "U.S. Deparement of Commerce

5285 Port Royal Road

Springfield, Virginia. 22151

ire: Pribred Copy \$5.45, ingerofiche \$ 1 t 


\section{MEASUREMENTS OF COSMIC RADIATION DOSE IN SUBSONIC COMMERCIAL AIRCRAFT COMPARED TO THE CITY-PAIR DOSE CALCULATION}

\author{
Roger Wallace \\ Lawrence Berkeley Laboratory \\ University of California \\ Berkeley, California 94720
}

July 16,1973

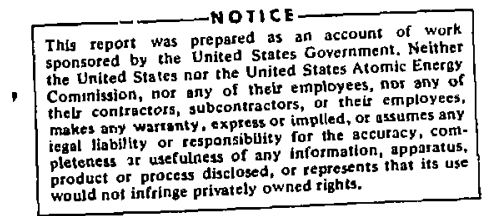

\section{INTRODUCTION}

The work for this project was carried out in three phases, as MS projects by two students, and as a postdoctoral project by a visiting scientist. The first phase was completed by Michael F. Boyer ${ }^{1}$ in 1969-70, and consisted entirely of measurements between San Francisco and London over the great circle route. The second phase was carried out in 1971-72 by Jon A. Kirby, over routes from San Francisco to Washington, D. C., Tokyo, Buenos Aires, and Rio de Janeiro. The City-Pair code, which originated at Boeing Aircraft Co., was reprogrammed to run on the computers at the Lawrence Berkeley Laboratory by Douglas C. Wallace, and the code was revised further by Kalina MamontCieśla, guest physicist, who is visiting the Lawrence Berkeley Laboratory from the Central Laboratory of Radiological Protection, Warsaw, Poland.

In the first phase, photographic emulsions and thermoluminescent dosimeters were mailed by conventional registered air mail from Berkeley via San Francisco to Professor Jack Fowler at Hammersmith Hospital near London, who returned them to us. These detectors accumulated approximately 2100 hours of flight time at altitudes above 30,000 feet. In the second experimental phase, similar detectors were mailed round trip through diplomatic channels to Washington, D. C., and thence to Tokyo, Buenos Aires, and Rio de Janeiro for a total flight time above 30,000 feet in excess of 2900 hours. The dates of the flights are listed in Tables $I a$ and $I I$, and of sola proton events in Table Ib. The 
measured results were then compared to the results of the "Boeing Code" which was originally programmed by Stanley Curtis and was originally described in the Boeing report. 2 This program, called the Galacti: Radiation Exposure Program (GREP), calculates the dose in tissue in millirads for the great circle flight path between two cities. The agreement is seen to be reasonably good.

It is concluded that both experiments and theory show that the total duses received at present day conventional jet aircraft altitudes are considerably higher than those encountered in supersonic flights at much higher altitudes, even though the dose rate is lower at these lower altitudes, when the longer time of exposure at the lower altitudes is taken into consideration.

\section{The Experiments}

The dosimetric measurements were made with thermoluminescent dosimeters (TLD's) and with emulsions of three types sealed in plastic packets. These packets were sent by air mail back and forth from Berkeley, California to the five cities on the dates shown in Tables Ia and II. A dose sufficiently above background for a satisfactory measurement was accumulated by the $T L^{2}$ s on one round trip and by the emulsions on three round trips. Although there were some small variations in the contents of certain packets, all were basically the same. Pieces of polyvinyl-chloride $(0.6 \mathrm{~mm}$ thick) were cut to the size of a regular business envelope $(10 \mathrm{~cm} \times 23 \mathrm{~cm})$. The packet was compartmentized and sealed with a radio-frequency plastic welder. Packets sent to London contained $\beta-\gamma$ films, NTA films, $600 \mu$ emulsion, and $\mathrm{CaF}_{2}$ thermoluminescent detectors (TLD). Packets sent to Washington, Tokyo, Rio de Janeiro, and Buenos Aires contained only $\beta-\gamma$ emulsions and TLD's. Before :ealing, each packet was flushed with dry nitrogen gas to reduce photographic fading of the latent image by decreasing the relative humidity and decreasing the atmospheric oxygen in contact with the emulsion.

Each packet contained four $\beta-\gamma$ films. Two of these films were 
unexposed; the third film was pre-exposed to $20 \mathrm{mr}$ and the fourth film was pre-exposed to $100 \mathrm{mr}$ of radium $\gamma$-rays. In those packets that contained nuclear track emulsions (NTA), one NTA film was pre-exposed to 20 mrem and the other to $100 \mathrm{mrem}$ of PuBe neutrors.

In evaluating the flight paths for the first part of this experiment from San Francisco to London, an air mail flight schedule was obtained from the Berkeley post office. When the total number of available flights is considered, it is reasonable to assume that at least $80 \%$ of the packets sent to London made the trip by the polar route, rather than lazding in New York. Polar flights from San Francisco to London always go via Los Angeles. On the Los Angeles to London leg they ave a flight profile approximately like that seen in Fig. 1. These flights usually go over the southern part of Hudson Bay, Baffin Island, and the southern third of Greenland. Each flight is flown over the predicted "least time" route based on the latest weather predictions. Some flights may go considerably south of Greenland, occasionally as far sou ih as Atlanta, although this is rare. These variations probably don't have a large effect on the galactic cosmic ray dose since they take longer at a lower dose rate, which has a compensating effect on the integrated dose. The solar flare dose, if any, would be reduced by a larger factor at the lower magnetic latitudes. Since few solar flares occurred during this experiment, these relatively rare and self-compensating route variations have little effect on our results.

Calculations made with the GREP code ${ }^{2}$ indicate that for the San Francisco to London route one should expect a total dose of about $5 \mathrm{mr} / \mathrm{round}$ trip. Since the lower limit of sensitivity for the film is approximately $10 \mathrm{mr}$, each packet to London was sent on about five round trips. Unfortunately, during this time there were no large flares and only one small flare. Three groups of packets completed five round trips to London. Six groups of packets completed round trips to Washington, Tokyo, and Buenos Aires. Five groups of packets completed round trips to Rio de Janeiro. 
$33,000 \mathrm{ft}$.

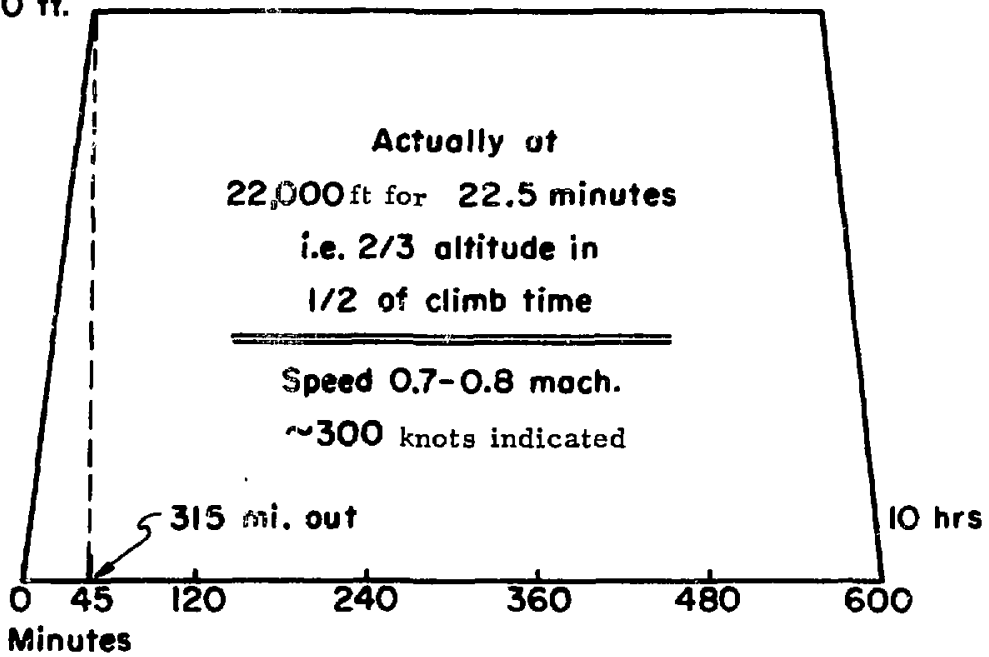

XBL713-3097

Fig. 1. Flight profile of a typical flight between Los Angeles and London from TWA. 
One packet was lost on this route.

The packets sent to Washington, Tokyo, Rio de Janeiro, and Buenos Aires actually followed the following routes, according to the U. S. Postal Service and the U. S. Department of State. The return route was the reverse of the sutbound route in each case. In the calculations, each leg of chese routes was assumed to be a great circle path between the two points at its end. The Washington route was direct from San Francisco to Washington. The Tokyo route was also direct from San Francisco to Tokyo non stop. The Rio de Janeiro route was from San Francisco to New York; to Charleston, South Carolina; to Carácus, Venezuela; to Rio de Janeiro, Brazil. The Buenos Aires route on the other hand, was from San Francisco to Washington, D. C.; to New York; to Buenos Aires, Argentina. The dates of the flights are shown in Table Ia and the dates of solar proton events are shown in Table $I \mathrm{~b}$. Background Radiation

Realizing that from the time the film is sealed until it is developed, it spends more time at sea level than at altitude, it is necessary to attempt to estimate the dose of ionizing radiation which is acc amulated during the time not spent in the aircraft.

Duplicate dosimeters were stored in a low background cave at the Lawrence Berkeley Laboratory anỏ in an unshielded area (Oakland) during the flight times. These dosimeters were processed with the dosimeters that had been mailed and the background readings were subtracted appropriately.

\section{Analysis of $\beta-\gamma$ Film Data}

In interpreting the data there were two experimental factors which need special mention. First, these films, all from the same emulsion number,were packaged, exposed, and developed in different groups; and secondly, the time which elapsed from loading to development in the different groups was different, even though the time which each group spent in the air was essentially the same. The total dose gathered ou 
Table Ia. The TLD's each flew on one trip between the following dates

\begin{tabular}{|c|c|c|c|c|c|c|}
\hline \multirow{2}{*}{ Washing:on } & \multicolumn{3}{|c|}{1971} & & \multicolumn{2}{|c|}{1972} \\
\hline & $\begin{array}{c}\# 1 \\
7 / 28-8 / 8\end{array}$ & $\begin{array}{c}\# 2 \\
8 / 28-9 / 2\end{array}$ & $\frac{43}{9 / 28-10 / 12}$ & $11 / 17-12 / 3$ & $\begin{array}{l}\text { \#5 } \\
1 / 13-1 / 20 *\end{array}$ & $\frac{\# 6}{4 / 6-4 / 14}$ \\
\hline Tokyo & $7 / 28-8 / 6$ & $8 / 27-9 / 9$ & $9 / 28-10 / 6$ & $11 / 17-12 / 9$ & $1 / 13-1 / 24 *$ & $4 / 6-4 / 17$ \\
\hline Buenos Aires & $7 / 28-8 / 18$ & $8 / 27-9 / 16$ & $9 / 28-10 / 19$ & $11 / 17-12 / 6$ & $1 / 13-2 / 1 *$ & $4 / 6-4 / 26$ \\
\hline \multicolumn{5}{|c|}{$\begin{array}{l}\text { TLD Dates Between } \\
\text { Stast and Reading }\end{array}$} & $1 / 13-2 / 16 *$ & $4 / 5-5 / 5$ \\
\hline \multicolumn{7}{|c|}{ The films each flew three trips as follows: } \\
\hline Tokyo & $\begin{array}{l}\# \mathrm{~A} \\
\# \mathrm{~B}\end{array}$ & $\begin{array}{l}1,2,3 \\
4,5,6\end{array}$ & & & & \\
\hline Buenos Aires & $\begin{array}{l}\# A \\
\# B\end{array}$ & $\begin{array}{l}1,2,3 \\
4,5,6\end{array}$ & & & & \\
\hline Rio d: Janeiro & $\begin{array}{l}\# \mathrm{~A} \\
\# \mathrm{~B} \\
\# \mathrm{C}\end{array}$ & $\begin{array}{l}1,3,4 \\
2,3,4 \\
4,5,6\end{array}$ & & & & \\
\hline
\end{tabular}

* There was solar activity during these flights, See Table Ib. 


\begin{tabular}{|c|c|c|}
\hline Date & $\begin{array}{c}\text { Strength } \\
\text { (all events low energy) }\end{array}$ & Duration \\
\hline September 1, 1971 & small & short \\
\hline October 3, 1971 & medium & day \\
\hline December 2, 1971 & small & short \\
\hline $\begin{array}{c}\text { December } 14-21,1971 \\
\text { peak December } 17\end{array}$ & medium to large & 7 days \\
\hline January 11,1972 & small & day \\
\hline January 15-16, 1972 & small & 2 days \\
\hline $\begin{array}{r}\text { January } 19-25,1972 \\
\text { peak January } 20-21\end{array}$ & medium & 6 days \\
\hline Apri1 17-18, 1972 & small & 6 hours \\
\hline
\end{tabular}


the London trips made by these films represents about 2052 hours of exposure at altitudes above 30,000 feet, as calculated from the flight profile in Fig. 1. The total time spent above 30,000 feet on the Washington, Tokyo, Rio de Janeiro, and Buenos Aires flights was 2897 hours. Fading

The question of the change of the latent image during the 8 to 16 weeks between loading and development is poorly understood. The controls which were kept in the shielded cave showed an apparent fading in the last two groups and an increase of background in the first group. Many possible explanations for this were examined and discarded. We are forced to attribute this inconsistency in the background film exposures to unexplained but occasionally observed variations in $\beta-\gamma$ film dosimetry. The film was all from one emulsion number and all films were treated the same. The assumption was made in the case of each group that, for the time period from loading into the packets until development, all films in the group underwent the same fading and background change. Therefore, the figures in Table $\amalg$ represent the dose readings in roentgens minus the actual dose reading from the appropriate control film which was kept in the low background cave.

In groups 2 and 3 the fading of the control films was more that might be expected, even though they were sealed in nitrogen and kept in the cave, which has a very constant environment. In some low dose films the fading was as much as 60 percent. This proved to be especially annoying in the case of the last group, where the average readings were higher than in groups 1 and 2. (See Table II)

The experience which has been gained over many years in reading $\beta-\gamma$ film indicates that the data are reasonable. It may not be possible to attach dosimatric significance to the measurements of any one film, but, in view of the large total number of hours which the film spent in the air, the average is probably significant.

Many possible explanations of the wide variation in measured doses were examined. It is quite possible that packages 9 and 13 may have 
Table II. Results from the $\beta-Y$ films making flight San Francisco-London in the packets.

\begin{tabular}{|c|c|c|c|c|c|c|c|c|c|c|c|c|}
\hline \multirow[b]{2}{*}{ Group } & \multirow[b]{2}{*}{ Pkg。 } & \multirow[b]{2}{*}{ Rd. trips } & \multirow[b]{2}{*}{ Hrs. in air } & \multicolumn{5}{|c|}{ Total dose in milliroentgen gained } & \multicolumn{3}{|c|}{ mr/round trip } & \multirow{2}{*}{$\begin{array}{c}\text { Group } \\
\text { Average }\end{array}$} \\
\hline & & & & Zero & Zero & 20 & 100 & Ave. & Min. & Max. & Ave. & \\
\hline & 1 & 6 & 108 & 23 & 26 & 30 & 6 & 21 & 1.0 & 5.0 & 3.5 & \\
\hline & 2 & 5 & 90 & 16 & 19 & 57 & 23 & 29 & 3.2 & 11.4 & 5.8 & \\
\hline \multirow{2}{*}{1} & 3 & 5 & 90 & 19 & 19 & 23 & 27 & 22 & 3.8 & 5.4 & 4.4 & \\
\hline & 4 & 3 & 54 & 11 & 11 & 15 & 19 & 14 & 3.7 & 6.3 & 4.7 & \\
\hline \multirow{6}{*}{$\begin{array}{l}\text { (9 Dec. } \\
69 \text { to } 9 \\
\text { Apr. } 70 \text { ) }\end{array}$} & 5 & 5 & 90 & 16 & 23 & 16 & 9 & 16 & 1.8 & 4.6 & 3.2 & \\
\hline & 6 & 5 & 90 & 30 & 30 & 33 & 37 & 33 & 6.0 & 7.4 & 6.6 & 4.7 \\
\hline & 9 & 5 & 90 & 228 & 228 & 62 & 202 & 180 & 12.4 & 45.6 & 36.0 & \\
\hline & 10 & 5 & 90 & 24 & 31 & 20 & 21 & 24 & 4.0 & 6.2 & 4.8 & \\
\hline & 11 & 5 & 90 & 24 & 31 & 18 & 24 & 24 & 3.6 & 6.2 & 4.8 & \\
\hline & 12 & 5 & 90 & 28 & 28 & 17 & 33 & 26 & 3.4 & 6.6 & 5.2 & \\
\hline \multirow{8}{*}{$\begin{array}{l}2 \\
(10 \text { Mar. } \\
70 \text { to } 29 \\
\text { Mar. } 70)\end{array}$} & 13 & 5 & 90 & 90 & 100 & 118 & 88 & 99 & 17.6 & 23.6 & 19.8 & \\
\hline & 14 & 5 & 90 & 28 & 32 & 9 & 20 & 22 & 1.8 & 6.4 & 4.4 & \\
\hline & $\cdot 15$ & 5 & 90 & 28 & 30 & 12 & 28 & 24 & 2.4 & 5.6 & 4.8 & \\
\hline & 16 & 5 & 90 & 30 & 31 & 14 & 18 & 23 & 2.8 & 6.2 & 4.6 & 10.6 \\
\hline & 20 & 5 & 90 & 37 & 47 & 89 & 90 & 66 & 7.4 & 18.0 & 13.2 & \\
\hline & 21 & 5 & 90 & 64 & 92 & 104 & 97 & 89 & 12.8 & 20.8 & 17.8 & \\
\hline & 22 & 5 & 90 & 107 & 121 & 149 & 164 & 135 & 21.4 & 32.8 & 27.0 & \\
\hline & 23 & 5 & 90 & 30 & 44 & 96 & 69 & 60 & 6.0 & 19.2 & 12.0 & \\
\hline \multirow{5}{*}{$\begin{array}{l}3 \\
(13 \text { Mar. } \\
70 \text { to } \\
14 \text { Jul. } \\
70)\end{array}$} & 24 & 5 & 90 & 89 & 160 & 186 & 193 & 157 & 17.8 & 38.6 & 31.4 & \\
\hline & $\cdot 25$ & 5 & 90 & 47 & 54 & 96 & 94 & 73 & 9.4 & 19.2 & 14.6 & \\
\hline & 26 & 5 & 90 & 100 & 107 & 142 & 164 & 128 & 20.0 & 32.8 & 25.6 & \\
\hline & 27 & 5 & 90 & 61 & 71 & 78 & 90 & 75 & 12.2 & 18.0 & 15.0 & \\
\hline & 28 & 5 & 90 & 92 & 100 & 128 & 97 & 104 & 18.4 & 25.6 & 20.8 & 19.7 \\
\hline \multirow{2}{*}{\multicolumn{3}{|c|}{ Total Averages }} & & & & & & Ave. & Ave. & Ave. & Ave. & \\
\hline & & & & & & & & 62.8 & 8.3 & 16.1 & 12.5 & \\
\hline
\end{tabular}

* Approximately 18 hours in air at altitude per round trip 
been exposed to a source of radiation somewhere along their trips. To suggest that all of group 3 is higher from this type of cause seems very unreasonable. There is, however, experimental experience that for unknown reasons, probably connected with environmental conditions, as much as $50 \mathrm{mr}$ per film might be due to activation of the silver grains by a so far unidentified process cther than radiation. This phenomenon has been known to occur in the processing of large groups of films in which,for unknown reasons,most of the films which left the photoprocessing lab apparently gained an abnormally large amount of radiation, while the control films that did not leave the laboratory showed nothing abnormal.

Although no large solar flares occurred during the experiment, an attempt was made to correlate enhanced solar activity with those packets which showed a higher dose. This was only mildly successful. For example, June 13 and 14 were the most active days since the beginning of the year. There were large class $X$ events. Packet 27 flew during this period and showed the highest dose of all packets for group 3 . Packet 23 also flew during this time pexiod and showed the lowest dose of group 3.

If the readings for the four days previous to the May 30, 1970 flare, made on the polar route to London, are averaged together using the amounts over the $4.0 \mathrm{mrad}$ background, one gets an average of $1.93 \mathrm{mrad} / \mathrm{round}$ trip. We assume that half of this dose was accumulated during each flight direction, or that on a no-flare trip the extra amount of radiation from flying is about 1 mrad. There was thus an increase of about $50 \%$ per round trip due to this flare.

Analysis of TLD Data

The TLD's (thermoluminescent dosimeters) were $\mathrm{CaF}_{2} \cdot$ Each reading is actually an average of three separate dosimeters contained in a small plastic disk. All readings and calibration of these dosimeters was done at the Lawrence Livermore Laboratory under the direction of D. E. Jones, R. E. McMillan, and G. L. Seibel, Hazards Control Group, 
and are listed in Table III.

Due to their greater sensitivity (down to $0.1 \mathrm{mrad}$ ) TLD dosimeters were sent on only one round trip before being read. Of special interest is the TLD sent on the 30th of May, 1970. It was in the air when the first proton event in $\mathbf{4 5}$ days occurred. Unfortunately, on this particular day a TLD was not sent via JFY airport, and so no comparison could be made between the pslar and lower magnetic latitude routes. However, the measurement during the flare was clearly above the other measurements. There was at most a $50 \%$ increase in dose for the flight during this class M flare.

\section{Experimental Results}

The experimental results from the first phase of the experiment are listed in Table $I I$. The average additional dose from cosmic rays on Elights to London from December, 1969 to July, 1970 was $12.5 \pm 4$ $\mathrm{mrad} / \mathrm{round} \mathrm{trip}$ with a lower limit of $8.3 \mathrm{mrad} / \mathrm{trip}$ and an upper limit of $16.1 \mathrm{mrad} / \mathrm{round} t \mathrm{ip}$. The results from the second phase of the experiment, including both the beta-gamma films and the TLD dosimeters,are summarized in Tables III to VI.

\section{Measured Cosmic Ray Neutron Spectrum}

One of the $600 \mu$ emulsions was scanned for proton recoils, and these in turn converted to the neutron spectrum in Fig. 2.

\footnotetext{
A description of the flare of May 30,1970, as given by ESSA, is as follow s:

"The proton event was associated with an imp IN, a Class $M$ flare at 30/0240Z, (IN-A size and intensity evaluation. In this case,area $2.1-5.1 \mathrm{sq}$. deg with normal intensity.) again in region 760 . The $1-8$ A $x$-ray burst associated with this flare had a peak flux of only 0.04 erg per sq $\mathrm{cm}$ per $\mathrm{cm}$ per sec,but a total duration of 6 hours. Protons were first detected by the ATS-1 satellite at about $30 / 0800 \mathrm{Z}$ and were of the order of 350 and 16 particles per sq cm per sec in the 5-21 and $21-70$ $\mathrm{MeV}$ channels respectively. Associated riometer absorption at $30 \mathrm{MHz}$ was I Db or less."
} 
Table III. CaF $\left.\operatorname{CLD}_{2} \mathrm{TL}^{60} \mathrm{Co} \mathrm{mrad}\right)$

Destination

Washington

Tokyo

Average

Buenos Aires

Average

Rio de Janeiro

Average

Cave Control

Home Control

(Oakland, Ca)

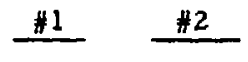

113

$\# 4$

$\# 5$

\#6

8.23

6.25

9.74

12.76

5.77

1.31

$\frac{8.34}{6.28}$

7.98

$\underline{12.47}$

9.86

12.62

$5.74 \quad 1.29$

5.76

1. 30

$\begin{array}{lll}7.45 & 8.38 & 10.26\end{array}$

14.20

6.80

2.28

$\underline{7.69 \quad 8.54 \quad 10.37}$

14.28

6.78

2. 32

14.24

6.79

2.30

7.82

2. 79

8.18

7.41

11.77

15.01

$\frac{7.86}{7.84}$

$\frac{2.92}{2.86}$

$\frac{8.63}{8.41} \quad \frac{7.71}{7.56} \quad \frac{12.05}{11.91}$

$\frac{15.35}{15.18}$

16.00

10.38

7.92

lost

$\frac{10.85}{10.77} \quad \frac{12.86}{12.57}$

$\frac{17.71}{16.86}$

$\frac{10.58}{10.48}$

7.87

16.86

7.90

Average

Average

2.75
$2.28 \quad 2.11$

2.71

$=\frac{2.13}{2.12}$

2.73

6.32

8.07

$\frac{7.15}{7.61}$

2. 37

2.07

0.25

$\frac{2,31}{2,34}$

2.15

2.11

0.21

$\begin{array}{lll}3.95 & 4.45 & 6.32 \\ 3.95 & \frac{4.52}{4.49} & \frac{7.53}{6.93}\end{array}$

6.93

2.34

0.26 
Table IV. $\beta-\gamma$ Films (226 Ra mr)

The doses shown are the excess dose above the pre-irradiated dose per individual film. Each film has been on three round trips. It should be noted that the averages were taken after excluding the $100 \mathrm{mr}$ exposed films, which apparently had experienced fading.

\begin{tabular}{|c|c|c|c|c|c|c|c|}
\hline & & 0 & 0 & 20 & 40 & 100 & Average/trip \\
\hline \multirow[t]{2}{*}{ Washington } & $\# \mathbf{A}$ & 29 & 30 & 30 & 30 & 17 & 10.0 \\
\hline & $\# B$ & 40 & & 36 & 31 & - & 11.8 \\
\hline \multirow[t]{2}{*}{ Tokyo } & $\# A$ & 30 & 29 & 26 & 30 & 13 & 9.8 \\
\hline & \#B & 41 & & 38 & 32 & & 12.3 \\
\hline
\end{tabular}

$\begin{array}{llllllll}\text { Buenos Aires } & \text { \#A } & 31 & 32 & 30 & 31 & 17 & 10.3 \\ & \text { \#B } & 43 & & 39 & 35 & & 13.0\end{array}$

Rio de Janeiro

$\begin{array}{lllllll}\text { \#A } & 39 & 36 & 31 & 30 & 8 & 11.3 \\ \text { \#B } & 36 & & 30 & 26 & & 10.2 \\ \text { \#C } & 54 & & 50 & 40 & & 16.0\end{array}$

Cave Control

$\begin{array}{ccccccc}\text { \#A } & 3.5 & 3.5 & 4.5 & 9 & -13 & 1.7 \\ \text { \#B } & 4 & & 2 & 0 & & 0.7 \\ \text { \#C } & 12 & & 13 & 0 & & 2.8\end{array}$

Home Controls

Average

\begin{tabular}{llllll} 
\#A (cold) & 11.0 & 11.5 & 14.5 & 20.5 & -1 \\
\#A(shelf) & $\frac{24.0}{17.5}$ & $\frac{24.0}{17.8}$ & $\frac{19.0}{10.8}$ & $\frac{22.0}{21.3}$ & $\frac{13}{7}$ \\
\#B(cold) & 30 & & 29 & 26 & \\
\#B(shelf) & $\frac{30}{30}$ & & $\frac{26}{27.5}$ & $\frac{22}{24}$ \\
\hline
\end{tabular}

Control for Ric de Jane iro flight \#B

The $100 \mathrm{mr}$ column is not included in the average. 


\section{Table V(A). Average dose/trip}

I'he doses shown are the average doses/trip above the background at Oakland, California. The values are secured, except as noted in the post note, by subtracting the Oakland, California values from the flight values shown in Table I.

TLD's (in Rad)

\begin{tabular}{lcccccccc} 
Destination & \#1 & $\# 2$ & $\# 3$ & $\# 4$ & $\# 5^{*}$ & \#6 & Net/Ave. \\
Washington & 4.34 & 1.78 & 2.93 & 5.01 & $3.22(7 / 34)$ & $0.72(8 / 30)$ & 3.00 \\
Tokyo & 3.62 & 3.97 & 3.39 & 6.63 & $4.00(11 / 34)$ & $1.60(11 / 30)$ & 3.87 \\
Buenos Aires & 4.46 & 3.07 & 4.93 & 7.57 & $4.55(19 / 34)$ & $1.60(20 / 30)$ & 4.37 \\
Riode Janeiro & & 6.28 & 5.64 & 9.25 & $6.76(26 / 34)$ & $6.64(25 / 30)$ & 6.91 \\
\hline
\end{tabular}

* These sets of TLD's were handled differently from the first four sets. These TLD's were kept in the cave except when in the mail. The TLD's were 34 (\#5) and 30 (\#6) days between preparation and reading. The numerator of the fraction in parentheses in the number of days it was out of the cave. The dose above background for these sets is found by:

Dose $=$ reported exposed dose minus cave dose minus (fraction) (home dose-cave dose)

This process takes into account the time the packets were in the cave. 


\begin{tabular}{|c|c|c|c|c|}
\hline & \#A & Film & $\# \mathrm{C}$ & Net/Ave. \\
\hline Washington & 4.0 & 2.7 & & 3.4 \\
\hline Tokyo & 3.8 & 3.2 & & 3.5 \\
\hline Buenos Aires & 4.3 & 3.9 & & 4.1 \\
\hline Rio de Janeiro * & 2.2 & 7.2 & 6.9 & 5.4 \\
\hline $\begin{array}{l}\text { * Trip \# A: Sin } \\
\text { 140/94 times th } \\
\text { background. }\end{array}$ & ntro & $\begin{array}{l}+46 \\
5 \text { us }\end{array}$ & $\begin{array}{l}\text { ger th } \\
\text { d the }\end{array}$ & $\begin{array}{l}\text { others, } \\
\text { are over }\end{array}$ \\
\hline
\end{tabular}


Table VI. Experimental and calculated equivalent doses received per round trip in conventional jet aircraft during average solar conditions between San Francisco and several cities.

\begin{tabular}{|c|c|c|c|c|c|}
\hline \multirow{2}{*}{$\begin{array}{l}\text { San Francisco } \\
\text { to: } \\
\end{array}$} & \multicolumn{3}{|c|}{ Calculated Solar Average Conditions } & \multicolumn{2}{|c|}{ Experimental } \\
\hline & $\begin{array}{l}\text { Charged } \\
\text { Particles }\end{array}$ & Neutrons & Total & $\bar{\beta}-Y$ Film & TLD \\
\hline London $*$ & 5.58 & 1.64 & 7.22 & 8.5 & 2.00 \\
\hline Washington & 2.39 & 0.68 & 3.06 & 3.4 & 3.00 \\
\hline Tokyo & 2.48 & 0.83 & 3.31 & 3.5 & 3.87 \\
\hline Buenos Aires & 6.98 & 1.74 & 8.72 & 4.1 & 4.37 \\
\hline Rio de Janeiro & 5.10 & 1.30 & 6.40 & 5.4 & 6.91 \\
\hline \multicolumn{6}{|c|}{$\begin{array}{l}\text { There was at most an increase of } 50 \% \text { during a class } M \text { solar flare which } \\
\text { cccurred on May } 30,1970 \text {, between San Francisco and London. }\end{array}$} \\
\hline
\end{tabular}


The proton recoil emulsion was tread using the random-walk method. Using this method, 1150 protoin recoil tracks were measured in the emulsion, which is approximately $2 \mathrm{sm} \times 2 \mathrm{~cm} \times 600_{\mu}$ in size. These data were then introduced into a computer prograrn which determines the track-length energy. The number of tracks per enezgy interval $\Delta N /(P . \Delta E)$ was then plotted versus energy. From this a smooth proton spectrum was drawn.

The two peaks at the low end of the proton spectrum are produced by systematic effects. They are caused by nitrogen in the emulsion (a nth, p reaction on nitroge $n$ ) and alphas from tharium and radium impurities.

Points from the smooth proton spectrum are then introduced into another program which determines the neutron spectrum. (See Fig. 2.) A second plot of this neutron spectrum was made with a linear scale. (See Fig. 3.) Then,using the relations shown below

\section{Analytic expressions for dose equivalent va neutrun energy}

\begin{tabular}{cc}
$\begin{array}{c}\text { Energy range } \\
(\mathrm{MeV})\end{array}$ & $\mathrm{n- \textrm {cm } ^ { - 2 } - \mathrm { sec } ^ { - 1 } \text { equivalent to }}$ \\
\hline$<10^{-2}$ & $1 \mathrm{mrem}-\mathrm{h}^{-1}$ \\
$10^{-2}-10^{0}$ & 232 \\
$10^{0}-10^{1}$ & $7.20 \mathrm{E}^{-3 / 4}$ \\
$>10^{-1}$ & 7.20 \\
\hline
\end{tabular}

An integral rem dose was calculated for each energy interval. This rem spectrum was then plotted with the linear neutron spectrum for comparison. (See Fig. 3.)

\section{Calculations}

The calculations ${ }^{2}$ were made by a code GREP originally programmed at the Boeing Aircraft Company by Stanley Curtis (now at the Lawrence Berkeley Laboratory) and modified by Douglas Wallace and Kalina MamontCiestla for this work. This code calculates tissue doses due to galactic 


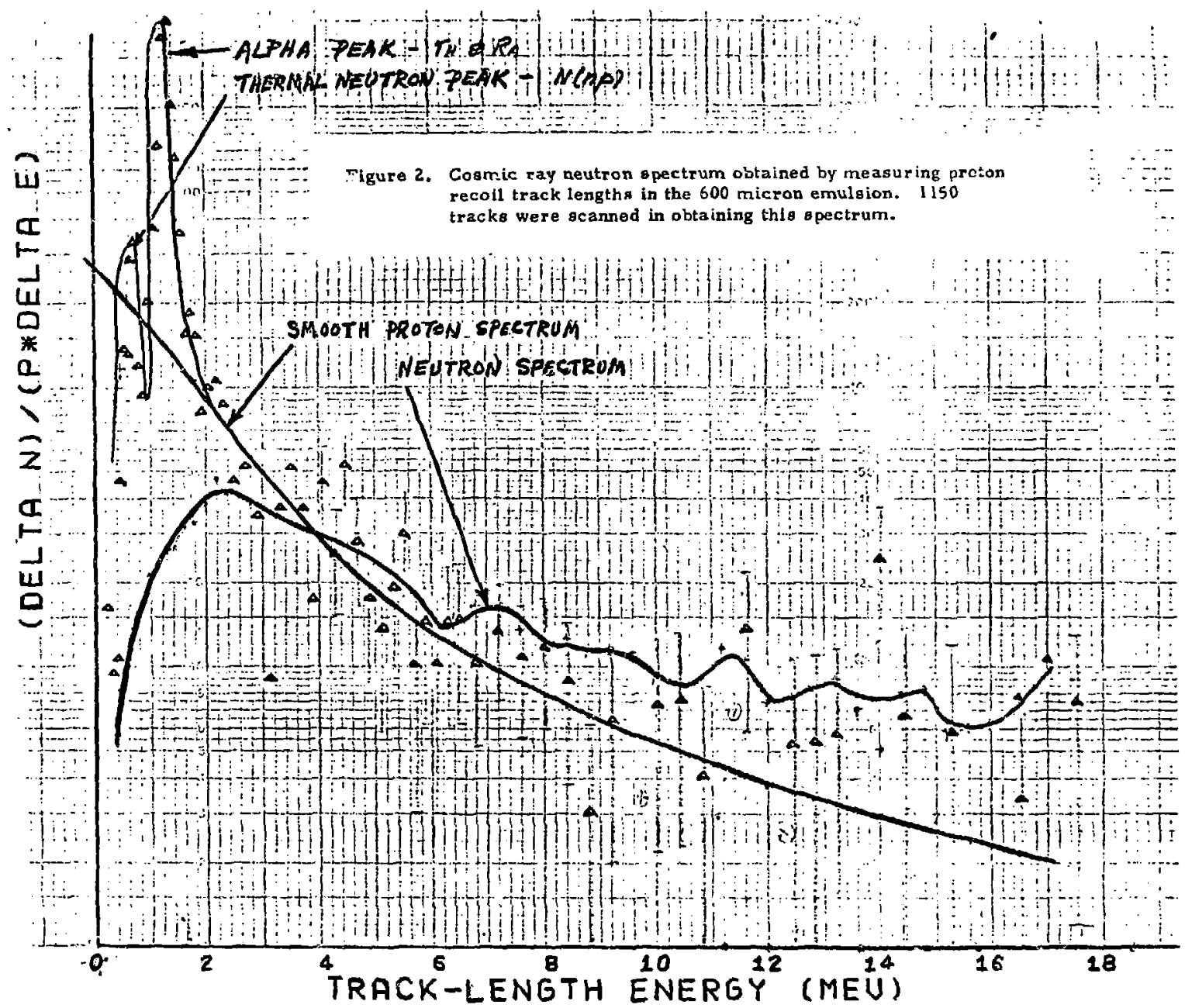




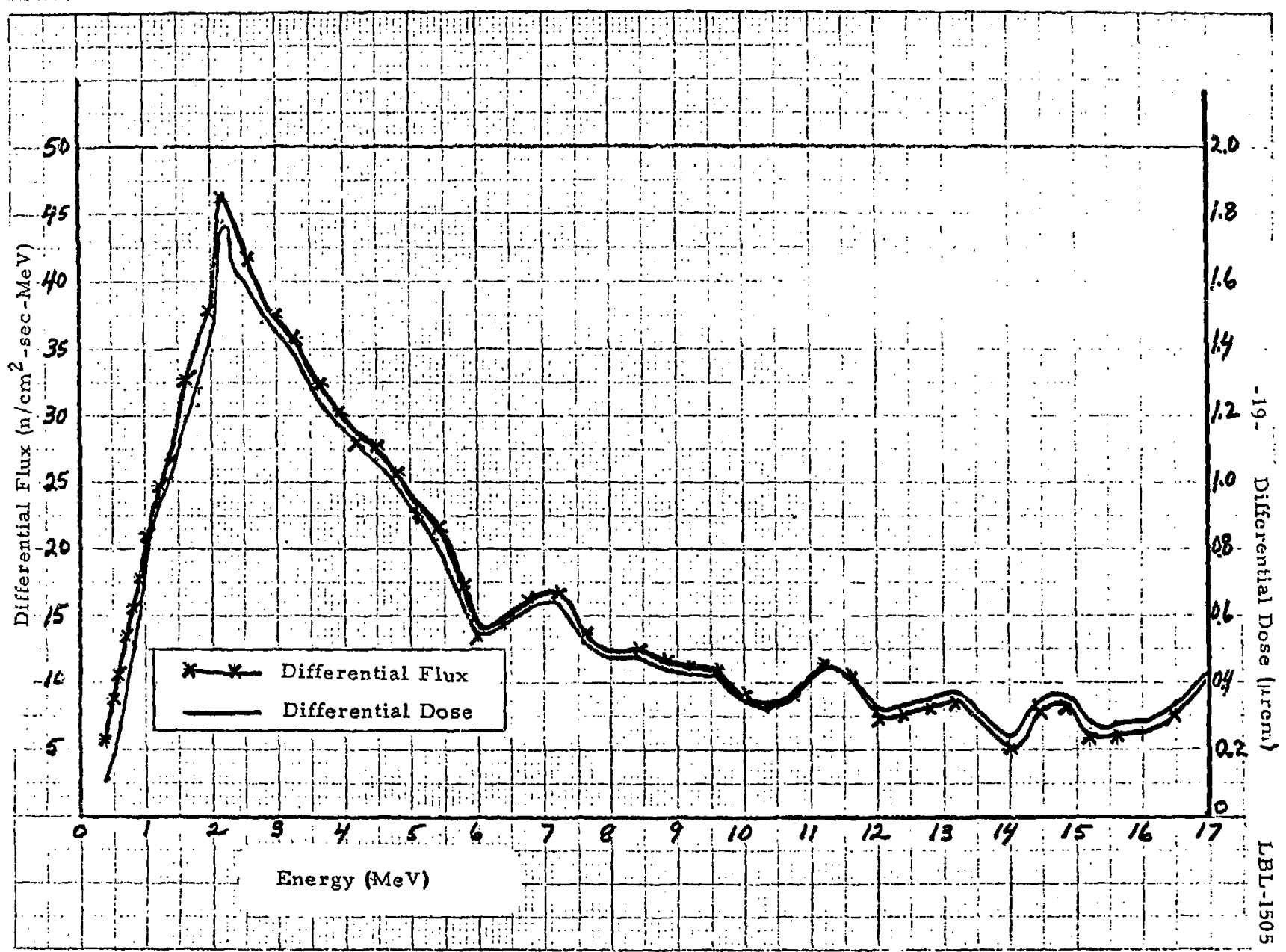

Figure 3 
cosmic radiation during subsonic and supersonic flight for times of minimum solar activity and times of average solar artivity.

In this calculation the input to the code, which is listed in the appendix, is the geographical coordinates of the cities, altitudedistance flight profiles, and block times. The program then changes the instantaneous geographic latitude and longitude of the plane to geomagnetic latitude and longitude and pressure altitude as it follows the aircraft on a great circle route. At 0.1 hour intervals, the ionization density, caused by charged particles in the atmosphere from basic cosmic ray data ion pairs formed in a $\mathrm{cm}^{3}$ per sec of standard air, is converted to an equivalent tis sue dose rate in ":rrads per hour with all the appropriate conditions taken into account. The ion pair data are eniered in a table from which values are interpolated as needed. This dose rate is then integrated and accumulated over the entire flight.

\section{Results}

In particular, note in Table VII that the direct Los Angeles - Paris flight is $3.70 \mathrm{mrad} /$ round trip for an SST under average solar conditions and that the same trip made by way of New York is $3.66 \mathrm{mrad} / \mathrm{round}$ trip. $(1.28+2.38=3.66$ ) In general, while more southerly routes have lower hourly dose rates, the flight routes are longer and thus more time is spent in these iower dose rate regions (due to the larger area of the earth in the equatorial and temperate zones). Thus, there is a compensating effect which tends to make doses on polar flights almost the same as those on lower latitude flights.

There is a similar compensating effect of altitude which takes place as altitude is varied. Subsonic flight at 35,000 feet takes about three times as long as supersonic flight over the same route at 65,000 feet. Since the dose rate is about three times higher at 65, 000 feet relative to 35,000 feet, these effects roughly cancel. As an example, 
Table VII. Comparison of the subsonic and supersonic round trip doses computed by the GREP code. The dose is given in mrem obtained when flying between various city pairs under average solar conditions.

City Pair

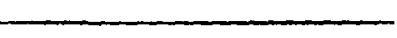

Paris-Anchorage

Los Angeles-Paris

Anchorage-Hamburg

Chicago-Paris

New York-Paris

Montreal-Paris

New York-Iondon

San Francisco-New York

Los Angeles-New York

Los Angeles - Washington

Los Angeles-Chicago

Sydney-Acapulco

City Pair

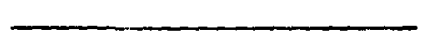

Paris-Anchorage

Los Angeles-Paris

Anchorage-Hamburg

Chicago-Paris

New York-Paris

Montreal-Paris

New York-London

San Francisco-New York

Los Angeles-New York

Los Angeles - Washington

Los Angeles-Chicago

Sydney-Acapulco $\frac{\text { Subsonic Flight }-35,000 \text { ft. altitude }}{\mathrm{mrad} / \mathrm{round} \text { trip }}$

(BT)

hrs

9.45

4.07

11. 15

4.79

8.95

3. 84

8.35

3.56

7.45

3. 13

7.05

2. 94

7.05

2. 94

5.45

2. 07

5.25

1. 92

4.95

1.75

3.95

1. 34

17.45

4.40

Supersonic Flight 60-65,000 ft. altitude Block Time mrad/round trip

(BT)

hrs

3.25

3. 16

3.85

3. 70

3.05

2. 92

2. 85

2. 64

2.65

2. 38

2.45

2. 17

2.45

2. 17

2. 05

1.44

1.95

1.28

1.85

1. 16

1.55

0.89

6.25

2. 08

$*$

Time in the air

* Two stopovers 
note that the Los Angeles to Paris SST dose is $3.70 \mathrm{mrad}$ and the subsonic dose is $4.79 \mathrm{mrad}$. In fact, the total doses for subsonic 35, 000 feet flights are between 25 and $100 \%$ higher than for flights at 65,000 feet, the supersonic altitude range. These increased doses in subsonic flight will undoubtedly be experienced by far more people than will experience the reduced doses associated with supersonic flights.

\section{References}

1. Michael Boyer, A Measurement of Cosmic Radiation Dose: Jet Aircraft to Polar Route, San Francisco to London, August, 1970. (M.S. Thesis.) Lawrence Berkeley Laboratory report, UCRL20052 .

2. City Pair Dose Calculations, Boeing Document D6 A11467-1. 
DIMFNS IIIN ALTT AB(300), DTAB (300), L TAB(1), TALTAB(15),

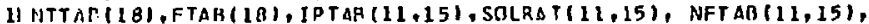

21. AMGOA:300), XI(300,3). TALT $(300)$, IDOSE $(300,2)$,

3 XDOSE 1300,4$)$ SPEED(3), MDOSE $(300,21, X$ XSEN $(300,4)$, TDOSE $(300,2)$,

4ALAT (2), PRBNMI2, 2), AIRPORT (800,B), ALONG $(2), T$ XOOSE $(300,4)$

LAMHDA,LING

IN:TFGER (DPRMM, IPPNRT ( 8DO,B), DIFER

EQUIVAI FNCE IIRPORT A AIRPIRTI

C* RAT READ IN FIXED DATA TABLFS. THFSE ARE THE SAME FOR ALL PROPLEMS.

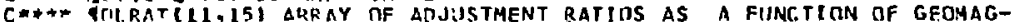

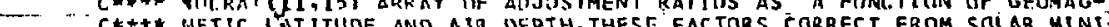

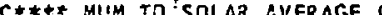

Cu*t NFTAH $(11,15)$ ARRAY IIF NFITTRON FLUXES AS a FUNCTION OF GEQMAG- IDF

CHAt NETIC LATITIUDE ANII AIR DFDTH

REAT $1000,(11$ PTAR $(1,3), J=1,15), 1=1,11)$ 11

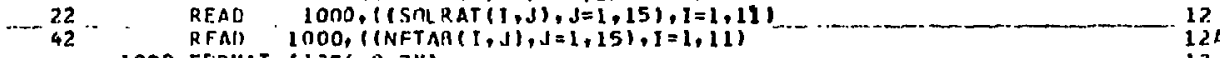

1000 FERMAT $(13 F G, 0,2 X)$

$C+\ldots+$

C*** TAITAB(15) ARRAY OF AIR DEPTH VALIIES $(G M / T, H * * 2)$

C***th LTAB $(1 \mathrm{~L})$ ARPAY OF RFOMAGNETIC LATITUDES

13A

C**** LNTTER(18) APRAY TF I OGAPI THHS OF THE MASS DF AIR OVERHEAD AT A- I3C

C*4.* GIVEN ALTITIDE IN FTAR

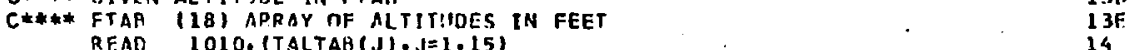

RTAD 1010, (LTAR(J),J=1,1I\}

READ 2010, (LNTTAB(J),J =1,18)

$\begin{array}{ll}\text { READ } & 1010, \text { (LNTTAB(J),J }=1,18 \\ \text { READ } & 1010, \text { (FTABI } J\}, j=1,18)\end{array}$

IOLT FORHAT I AF 10.0)

$C * * *$

C*** END OF FIXFก DATA TABLES FOR COSHIC RAY FLUXES AND AIR PROPERTIES.

C.*\#** PRINT DUT THESE FIXED DATA TABLES.

112 PRINT IIO, (TALTAR (J), J=L,15)

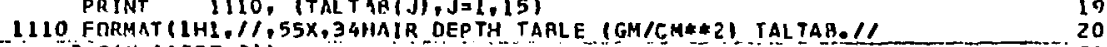

$2,24 \times,(15 F T$. O)

120

PRINT 1090, (ILTABII), (IPTAB(I,J),J=1,151), I=1,11)

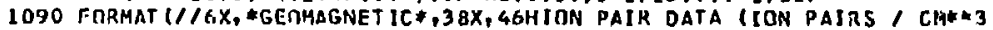

$1 / 5 F C$ (IPTAB,

$2,6 \times, * L A T$ I TUDF * 11

$3(6 x, f 5,0,13 x, 15+7,1 / 1))$
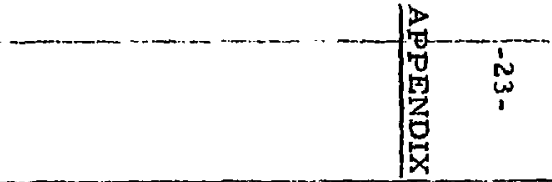

14

15
16
17

18

3

A

.

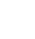

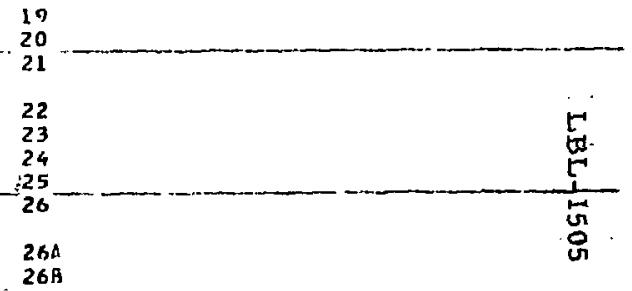




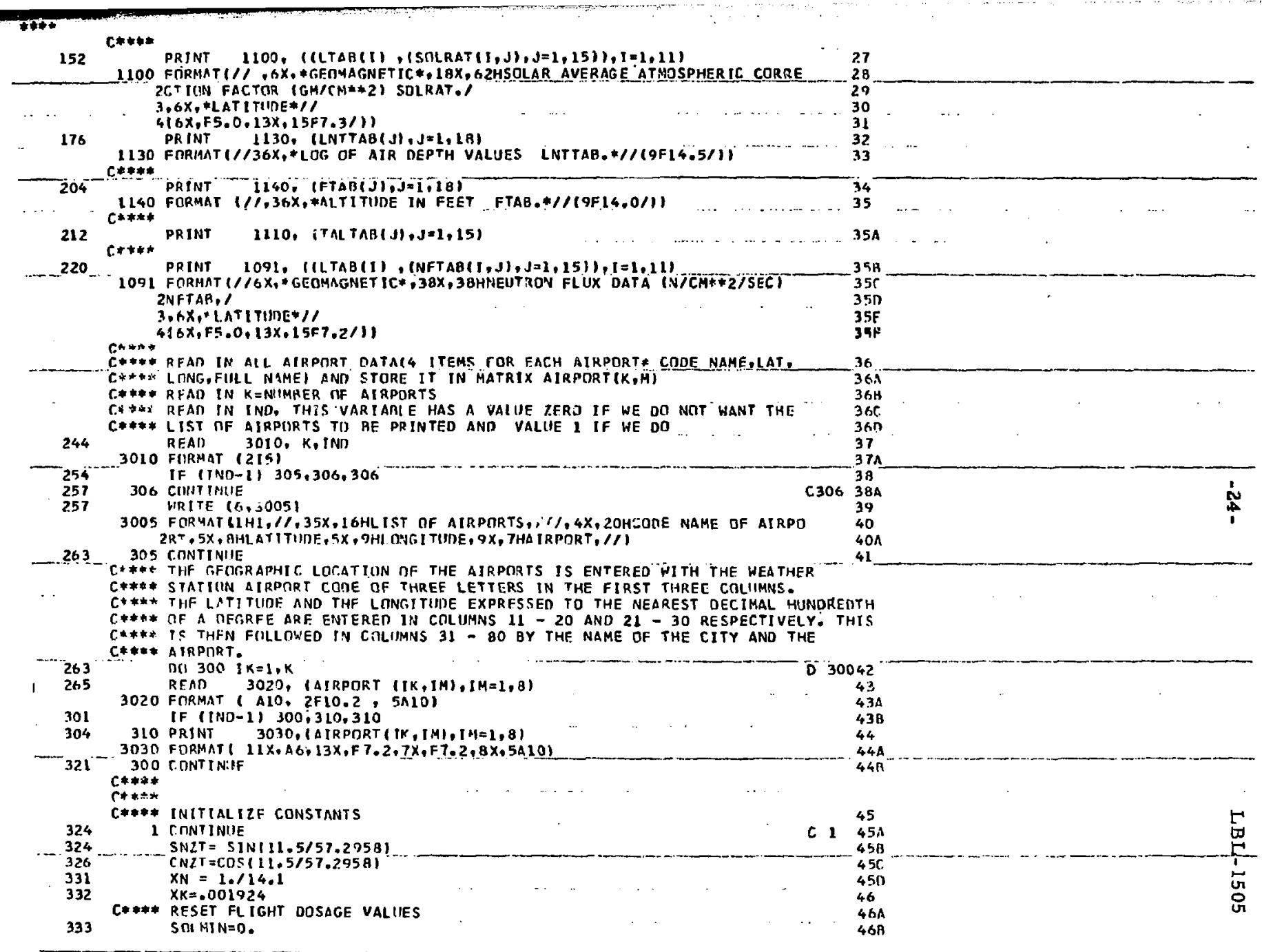




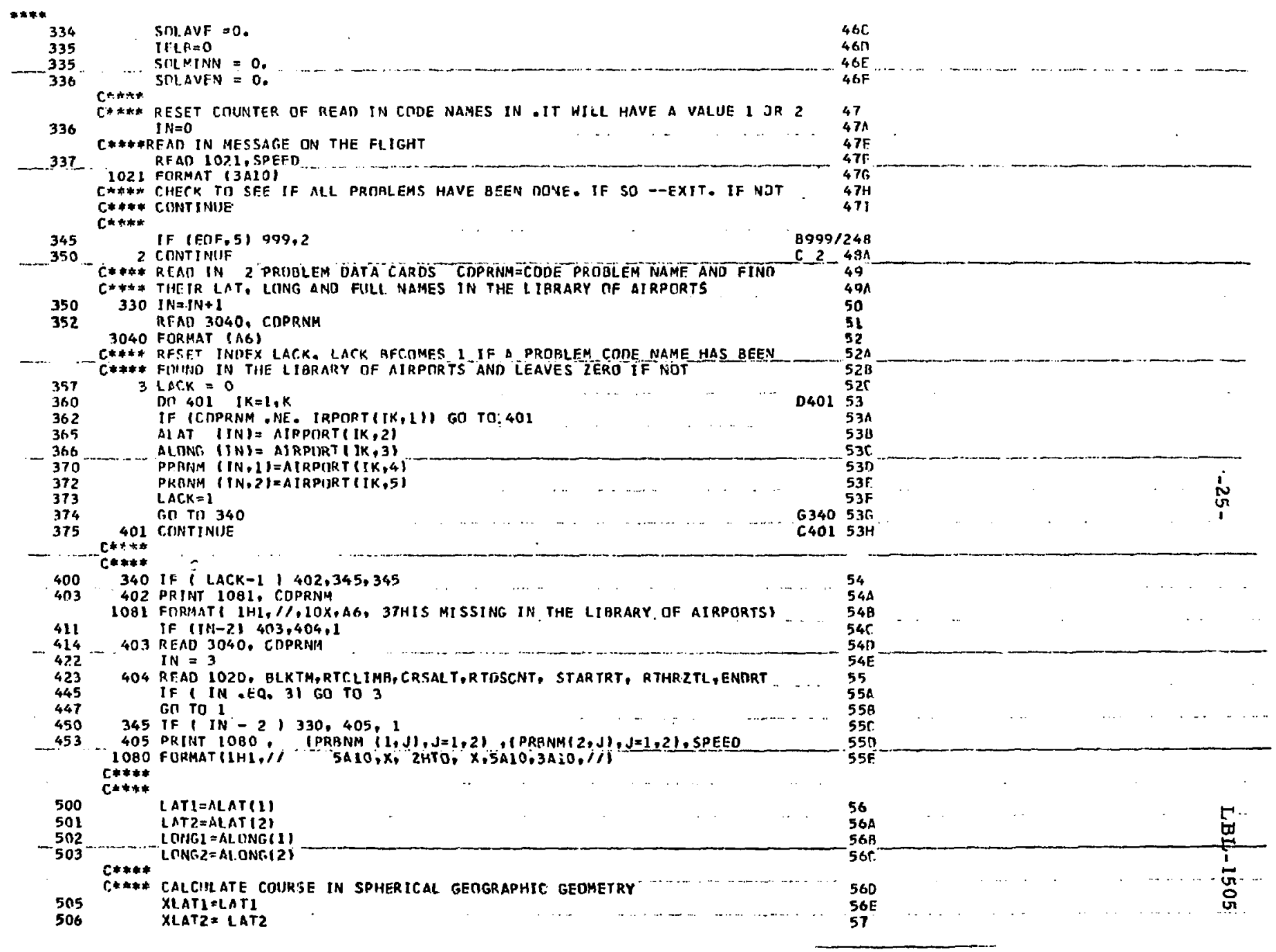



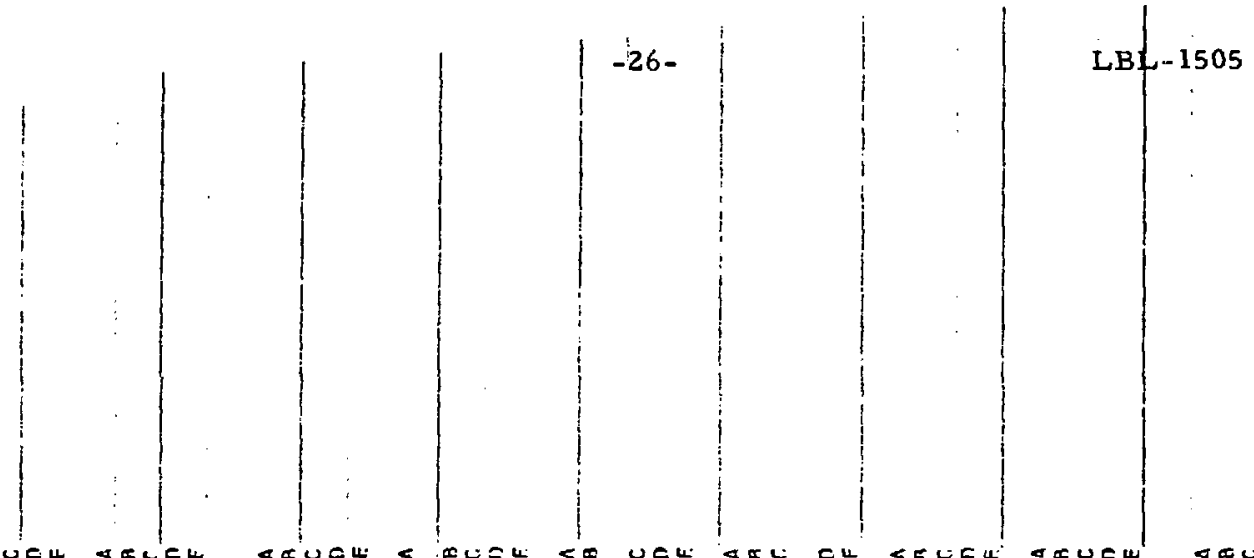

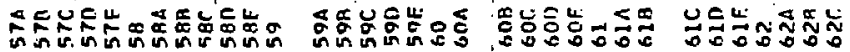

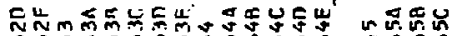

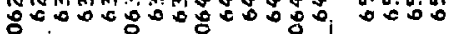
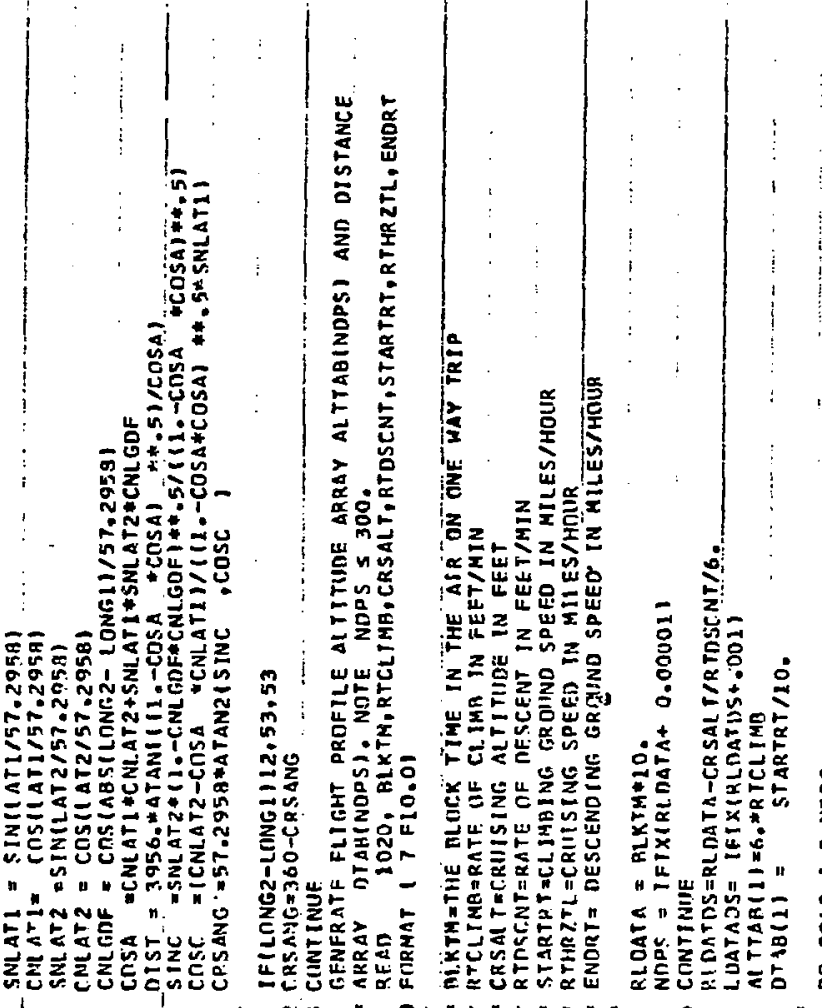

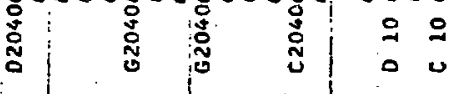

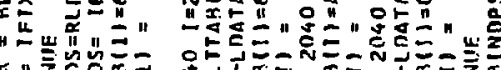

(1)

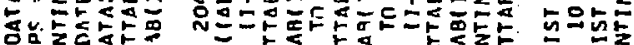

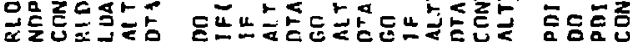

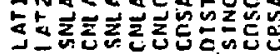
-

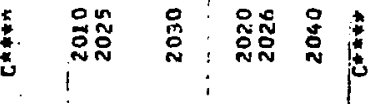

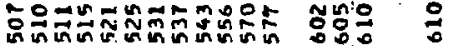

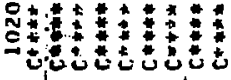


C+HA COMPARE THF GRFAT CIRCIE OITANCF DIST NITH THF DISTANIE PDIST CAL -

C.*** CIII ATFD AS A SII, BF GENERATFD DISTANCES TRAVFLLED. PER O.I HOLR

C* * INCRF.HENT AND CUMRECT THE REND IN SCHEDIILE VALUE OF BLKTM TO BLK

C.*\#*: IF NETFESARY

DIF = IDIST - POIST / /RTHRZTL /10.,

IF (AESIDIF) :LT, l, GT TO 102

DIFER $=$ IFIX I DIF i

IF ( DIFER - 0 I 101, 102, 103

101 NDPS $=$ NIPPS + DIFFR RLOATA = FLILAT N NDS GD TO 104

103 NDPS = NDPS - DIFFR RLNATA = FLOAT I NDPS Gก TII 104

- 102 CONTINIIE

PRINT IIAO, ALKTH,RTCLIMR,CRSALT, RTOSENT, STARTRT,RTHRZTL, ENDRT

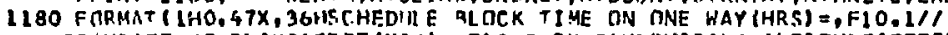

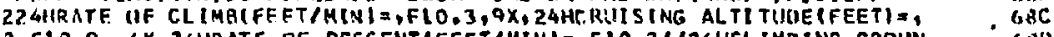
3 F10.0, 4X,26HRATF OF DESCENT IFFET/MINI=,F10,3//34HCLIMRING GROUN GAI)

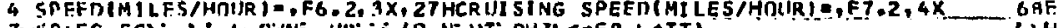

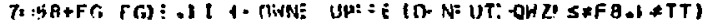

766 $C * * * *$
PRTHT 1150.
(AI.TT $A B(I), T=1, N D P S)$

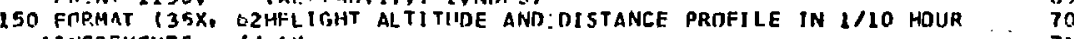
1INCREMFNTS. $1 / 4 \mathrm{X}$.

2 AHALTITUIE $/ /($ TF 10.011

PR.INT 1160, IOTMBIII, I=1, NOPS

1160 FORMAT (LHO, 3X, BHDISTANCE), BF 10.2 ) BIK = KIDATA $/ 10$. PRINT 1170, PDIST, BLK

1170 FORMAT (1H0, 3X, 3OHTOTAL INPIIT PRDFILE OISTANCE = "F 10.2 12X, 13IISTATIITE MILES $/ / 4 X, 19 H F L$ IGHT PROFILE TIME, $10 X, 1 H=, F 10.2$ $2,2 X_{C} 16$ HHIIJKS IN THE AIR ।

C**** PRINT OIT GREAT CIRCLE PATH VALIIES. PRINT 1050, OIST.CR.SANG, XLATL, L ING I, XLAT2, LONG2 GGG.

1004

1006

1026

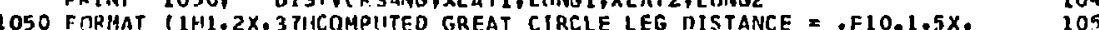
127HTHF INITIAL COIIRSE ANGLE $=, F 10.318$

$23 X, 21$ HLFG START LATITIIDF $\equiv, F 7 \cdot 2,5 X+22 H$ LEG START LLONG ITIIDE___. 3F 7.2.1/3X, LHHLEG FND LATITIIDF $=.3 x_{7}$

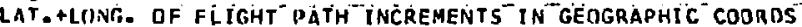

C**** NDTE THAT THE MIDPNINT DF THE 0.1 HDUR INTERVAL IS IISED TO COMPUTE II5

C**** PIISITINN ANU DIOSE.
IF (IFLA -GT. II GU) TO 15
B $\quad 15117$

1042 $x=0,5$ T GO TO 20 
C*\#\# CONVFRT TO MILLIRAISTHOIR

C. $\$ *$ IOTSE(NDPS,K) ARPAY GF INCRFMENTAL DOSE RATES FROH CHARGEO PAR- 15GA

C*** TICIES AND GAHMA RAY

C*** NOIISE (NDPS,KI ARRAY GF INCREMENIAL DDSF RATES FROH NEUTRONS - - ISGC

C* *t $K=1$ COIRRESPINOS TU THE SOLAR MINIMUM CONDITION

C**** $K=2$ CORRESPONDS TO THE SILAR AVERAGE CONDITION I56F

$=1364$ IONSE $(1,1)=$ IPAIRS $X K$

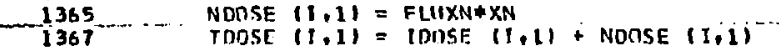

C* * XDOSE (NDPS, J) FOR CHARGF PARTICLES AND GAMMA

C**** XOOSFNCNOHS,J) FOR NEUTRONS

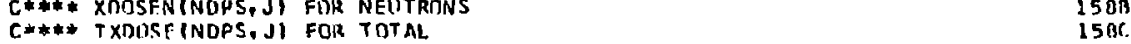

C*** J=1 CIRRESPONOS TO INCREMENIAL DOSE FOR THE SOLAR MINIMIJM CONDITION ISBD

C***Y J=? COPRESPIINIS TU CUMIILATIVE DOSE FOR THE SOI AR MINIM. CONDITION - 1.5BF

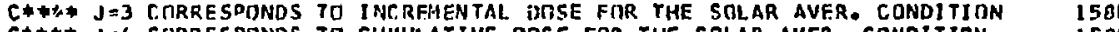

C**\#, I34 CIIRRESPONDS TO CIMIILATIVE DOSE FIR THE SILAR AVER. CONOITION

2371
1372

159 XONSFN(T,1)= NDISF $(1,1) \star 0.1$

TXDISE $(1,1)=$ XOUSF $(1,1) *$ XOOSEN $1,1,1$

IF I - C, T, II GO Ti 80

XIDSE $(1,2)=$ XDDSE $(I, 1)$

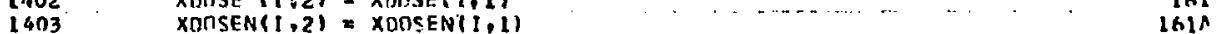

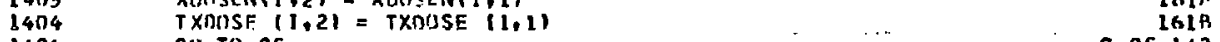

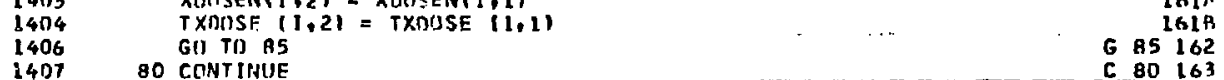

1509

$\begin{array}{rl}8 & 60160 \\ 161 & \end{array}$

1407

1411

XDOSE $(1,2)=\operatorname{xnOSF}(1,1)+\operatorname{XIONSE}(1-1,2)$

XIOSEN $(1,2)=$ XOOSEN $(1,1)+$ XDOSEN $(1-1,2)$

TXMISE $(I, 2)=$ XODSFN $(1,2)+\operatorname{XOOSE}(I, 2)$

6 A5 162

141785 CONTINIIF

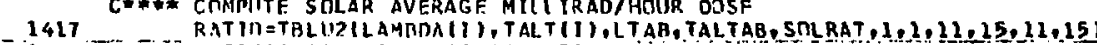

$-1427$

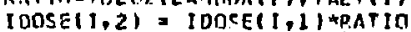

$\begin{array}{ll}1436 & \text { NOUSE }(1,2)=\text { NDOSE }(I, 1) \text { TRATIO } \\ 1440 & \text { TONSF }(1,2)=\text { TISTSII, I SRATIO }\end{array}$

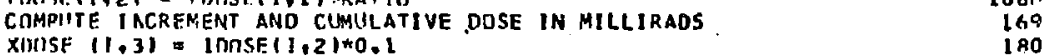
XIUUSF $(1,3)=1$ INTSE $(1,2) * 0.2$

1441 XNOSEN $(1,3)=$ NDUSE $(i, 2) \div 0.1$

-. 1443

1445

1447

1454

1455

1457

$-\ldots 1461$

14111

1463

1464

1467

1471

TXOMSE $(1,3)=$ XDUSE $(1,3)+$ XIOCOSENT 1,3$)$

IF II

XDOSE $(1,4)=$ XOUSE $(1,3)$

$\operatorname{XDISEN}(1,4)=\operatorname{XDOSEN}(1,3)$

TXDOSF $(I, 4)=$ XDISF $(I, 4)+X$ XOSEN(I,4)

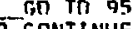

CONTINIE

$\operatorname{XIOSFN}(1,4)=\operatorname{XOOSEN}(1,3)+\operatorname{XODSFN}(1-1,4)$

$\operatorname{TXDOSE}(1.4)=\operatorname{XDNSE}(1.4)+\operatorname{XDOSEN}(1.4)$

95 CONTINIIE

100 CMNTINUF

85165

166

168

1684

$190 A$

B 901 ก1

182
$18: 2$

$18 \div \mathrm{n}$

G. $95 \quad 183$

90184

1854

$185 \mathrm{H}$

95186

C 100187

$C+4+4$

C**** PRINT QUT LEG INCREMENT RESILLTS

$18 B$

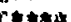

1474

PRINT

1060, $($ XI 1,1$), X I(1,2), X I(1,3), L A M B D A(I), T A L T(I)$, 
1IDOSE $(1,1), 1$ DOSE $(1,2)$, NDOSE $(1,1)$, NDOSF $(1,2), 1^{\circ}$ TONSE $(1,1), 189 \mathrm{~A}$

2TDOSE 11,2$\}, 1=1$, NOPSI 190

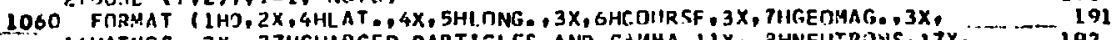

16HATHOS. . 3X, 27HCHARGED PARTICLFS AND GHAHA, $11 X_{1}$, GHNEUTRONS, $17 X_{0}, 192$

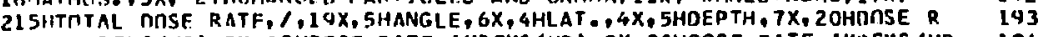

3ATF (MREMS/HR), 9X, 2OHDDSE RATE (MREMS/HR), 9X, 20HONSE R.ATE (HREYS/HR 194

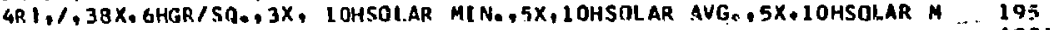

5IN.,5X, $195 \mathrm{~A}$

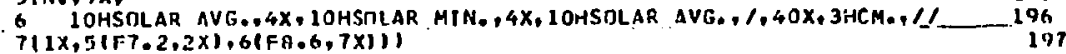

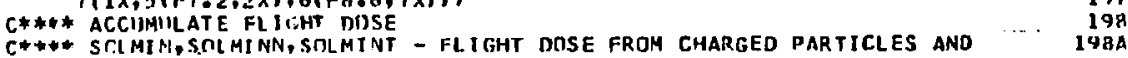

C*** GAMMA RAYS. NEUTRONS, BOTH FIR SOLAR MINIMIIM CONOITIONS 19BA

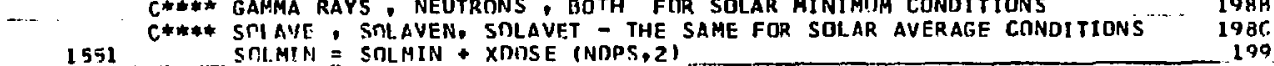

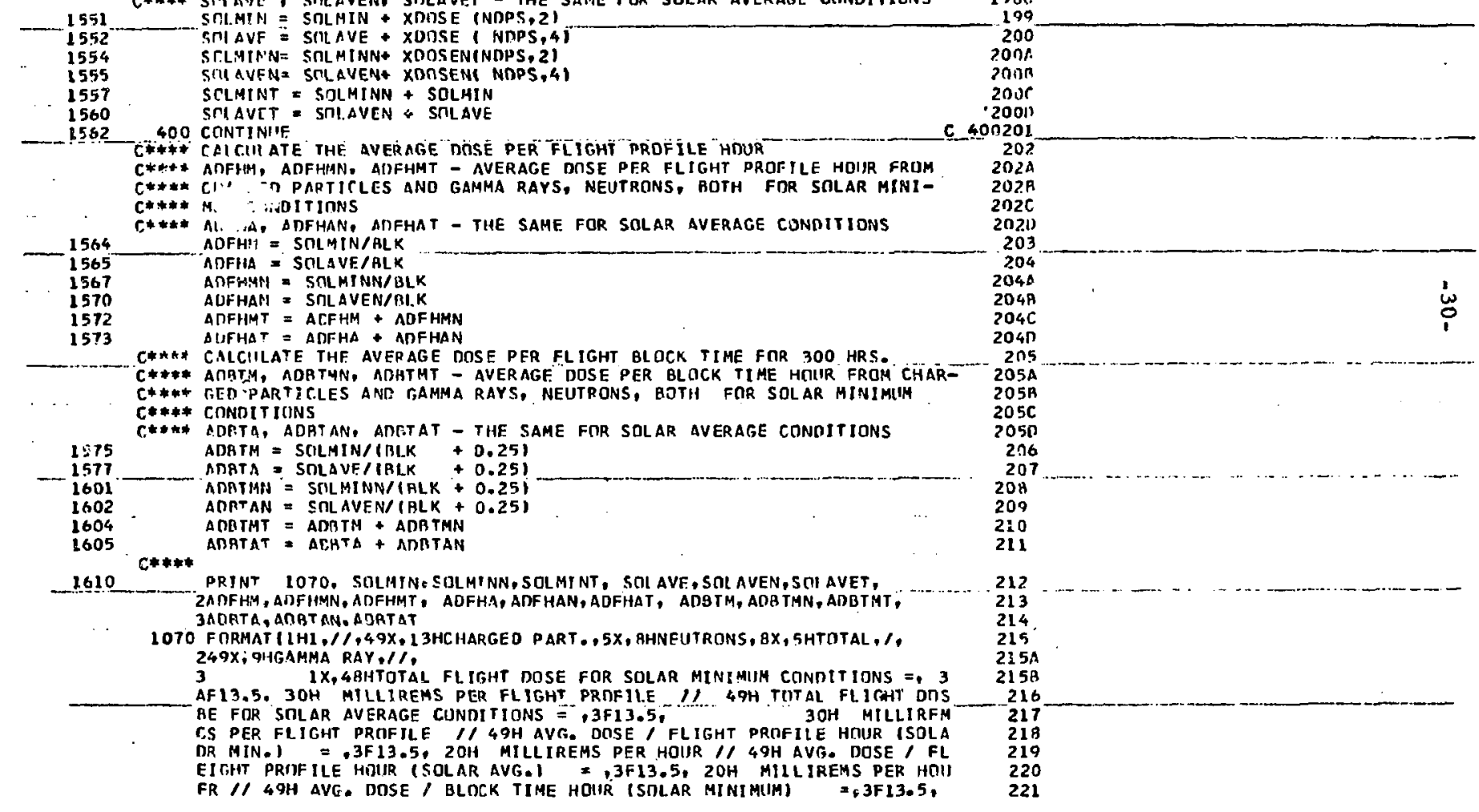

C**a* CALCIILATE THF AVERAGE DOSE PER FLIGHT BLDCK TIME FOR 300 HRS.

A

PRINT LOTO, SOLHIN: SULMINN,SOLMINT, SOL AVF, SOL AVEN,SOI AVET,

2ADFHM, ADFHMN, ADFHMT, ADFHA, ADFHAN, ADFHAT, ADBTM, ADB TMN, ADBTMT,

FORHAT I LH1,//,49X,13HC

3 IX,4BHTOTAL FLIGHT DOSE FOR SOLAR MINIMIIH CONDITIONS $=3$ AF 13.5. 30H MILLIREMS PER FLIGHT PROFILE II $49 \mathrm{H}$ TOTAL FLICHT DOS BE FOR SOLAR AVERAGE CUNDITIONS $=3 F 13.5, \cdots$ DR MIN.) $=$, 3F 13.5, $20 \mathrm{H}$ MILLIREMS PER HOUR // 49H AVG, DOSE / FL 
G31H MILLIREMS ' (AL TCK TTME) HIUR // 49H AVG. ODSE / RLOCK TIME H HOIR (SMLAR AVFRAGE) = 3F13.5,32H MILLIREMS / (ALOCK TIME) HD!J C*****

IR, IIX. 4ZHIEG BL DCK TIME = PRAFILE T ME + 0.25 HDURS,

$C+*+*$

SFE IF THERE IS ANOTHER RRMALEM. IF SO --PROCEED, IF NOT EXIT. GIT TO 1

1657
1660

1661 END

$6 \quad 232$

E 999233

PROGRAM I ENGTH INCLUDTNG T/O BUJFFERG

40227

STATEMENT FUNCTION REFFRENCËS

LICATION GEN TAG SYM TAG REFERENCES

STATEMENT NIIHAER REFERENCES

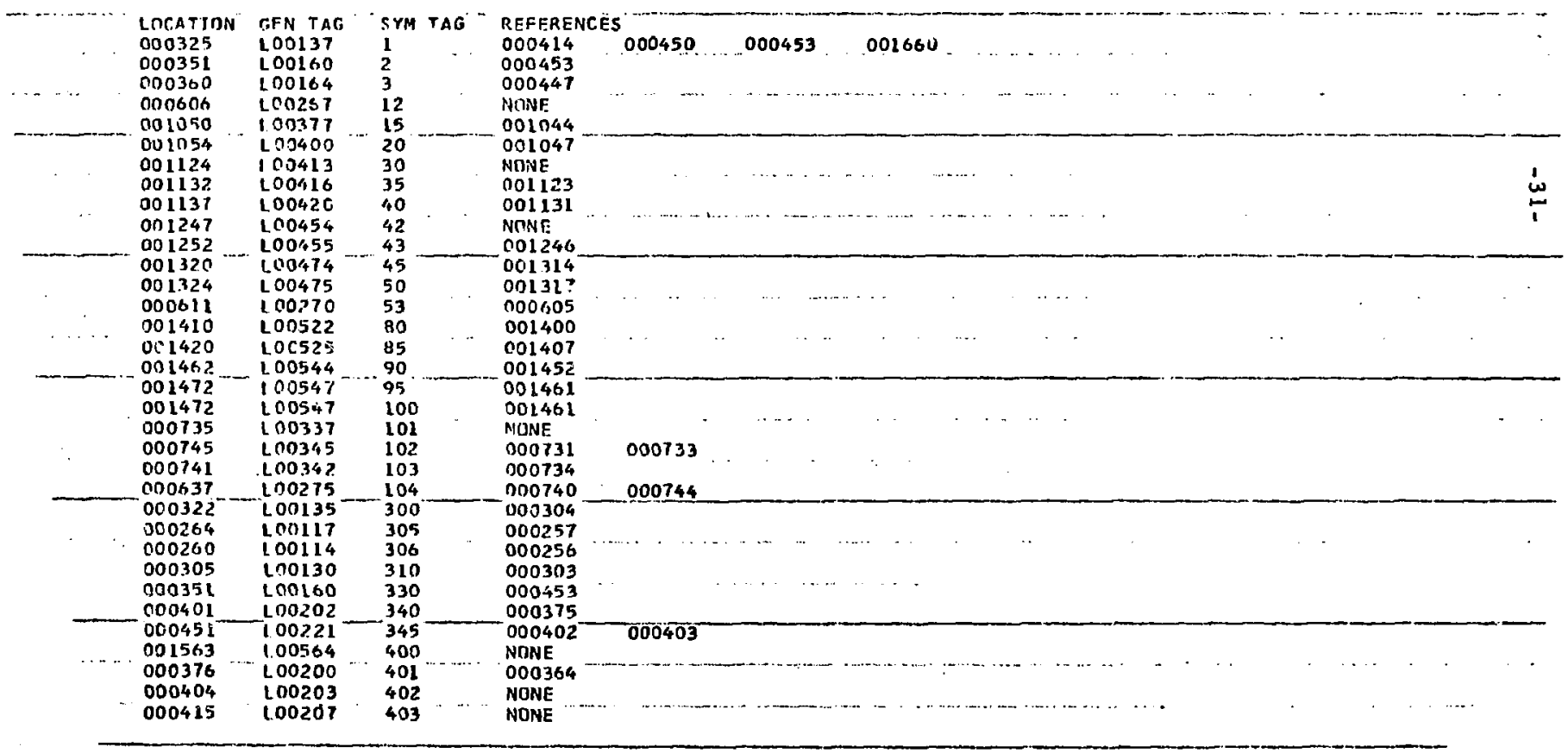




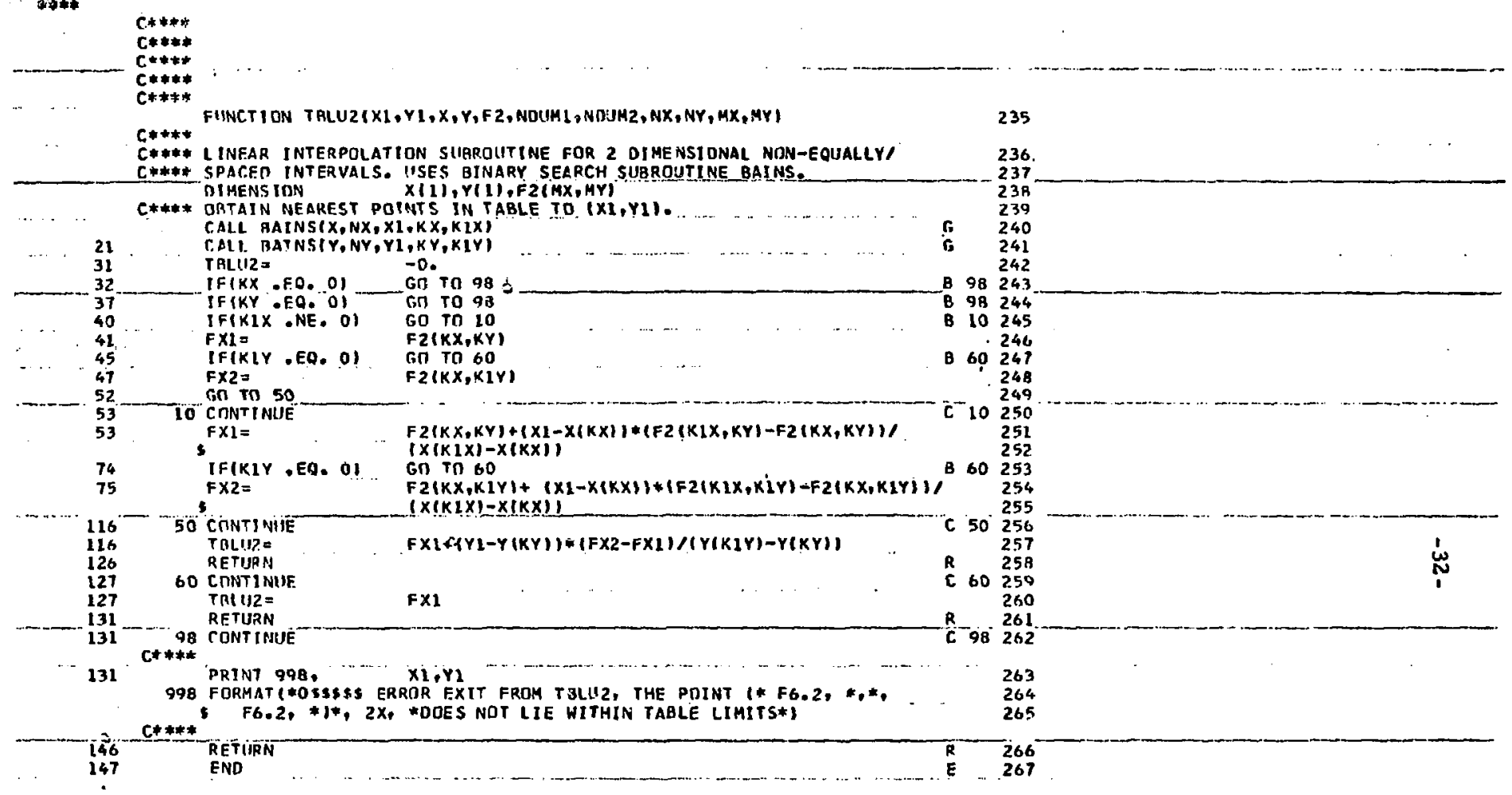

SUBPROGRAM LENGTH

00226

STATEMENT FUNCTION 'REFERENCES

LOCATION GEN TAG _SYM TAG REFERENCES

STATEMENT MIMAER REFERENCES

LOCATION GFN TAG SYM TAG REFERENCES

000054 L00037 10 OOOO41




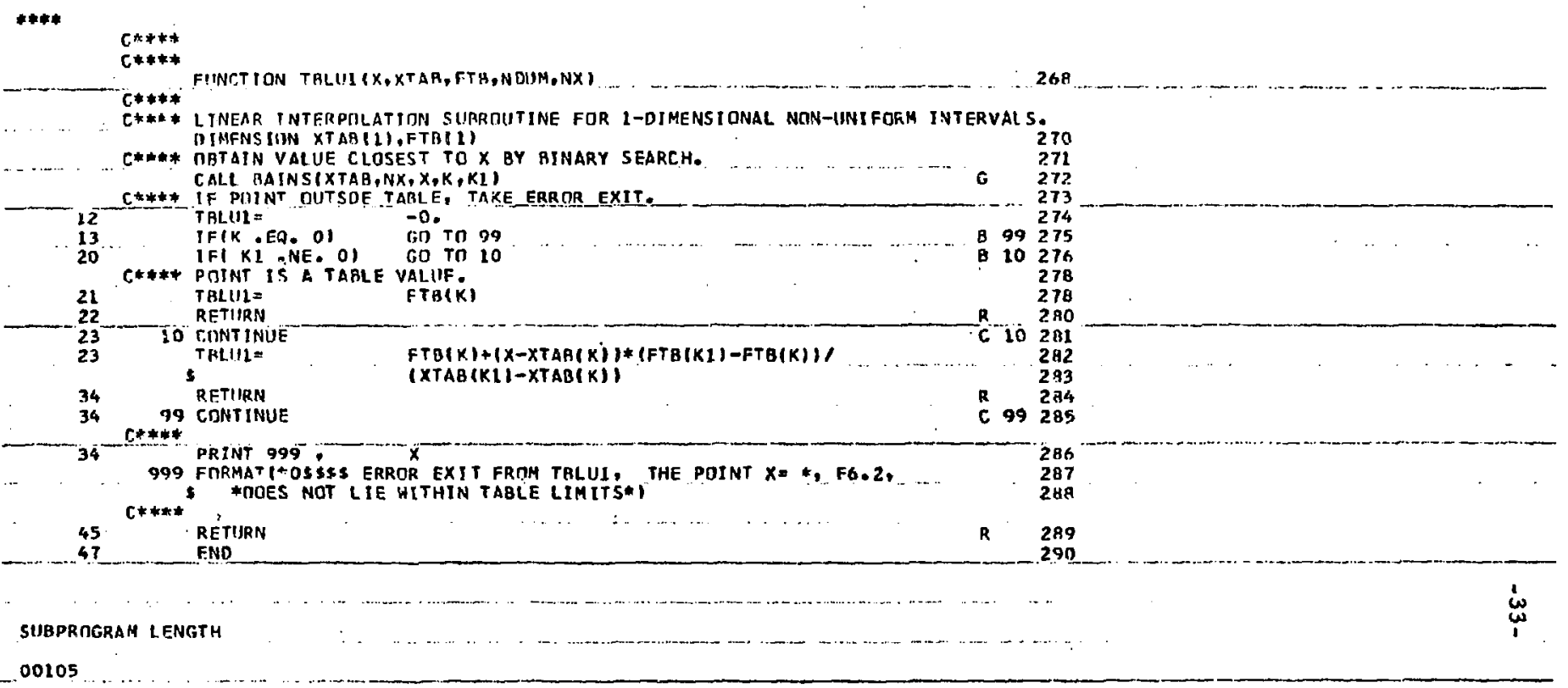

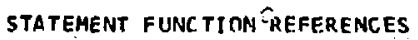

LOCATION GEN TAG SYM TAG REFERENCES

STATEMENT NIMABR REFERENCES

\begin{tabular}{llllll} 
LDCATINN & GEN TAG & SYM TAG & \multicolumn{2}{c}{ REFERENCES } \\
000024 & LO0022 & 10 & & 000021 \\
000035 & L00024 & 99 & & 000020 \\
000054 & C00004 & 999 & $\cdots$ & .000035
\end{tabular}

- BLock names añ "LENGTHHS

VARTABLE REFFRENCES

LICATINN GEN TAG SYM TAG REFERENCES

$\begin{array}{llllll}000103 & V 00107 & K & 000010 & 000017 & 000024 \\ 000104 & V 00010 & K i & 000011 & 000020 & 000025\end{array}$

$\begin{array}{llllll}000102 & \text { V00006 } & \text { KRLU1 } & 000011 & 000020 & 000025 \\ & 000017 & 000022 & 000034\end{array}$

000046

START DF CONSTANTS-000052 _.... TEMPS--000071 INOIRECTS-000101 
AIR DEPTH TABLE (GM/CM**2) TALTAB.

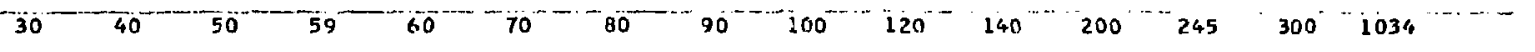

INN PAIR DATA IION PAIRS / CM\#\#3/SEC) IPTAB

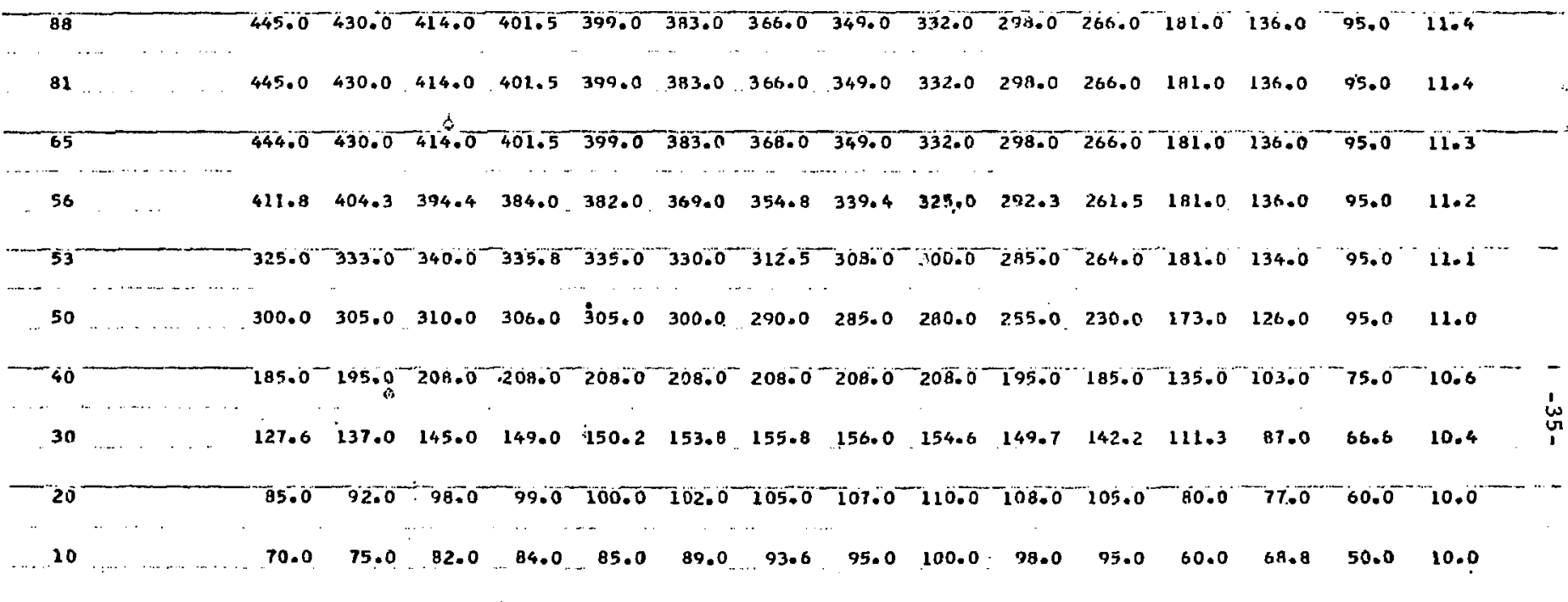


AIR DEPTH TARLE (GM/CH*\%2) TAL.TAB.

\begin{tabular}{|c|}
\hline-30 \\
\hline
\end{tabular}

GEOMAGNFTIC

NEUTRON FLlIX DATA (N/CM**2/SEC) NFTAB

LATITUDE

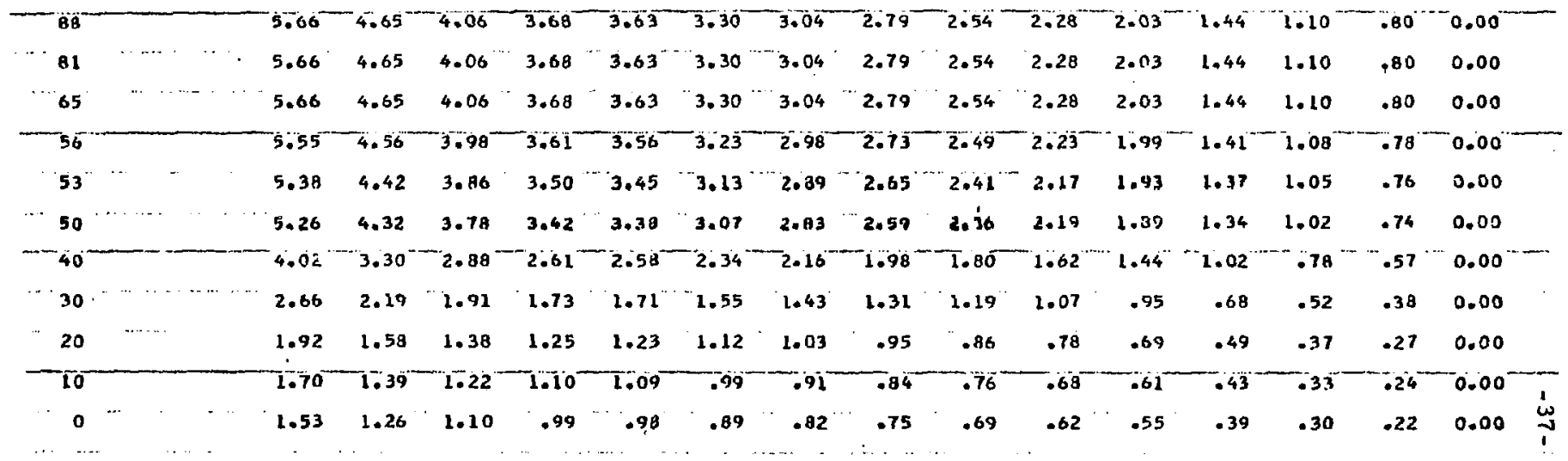


LIST OF AJRPORTS

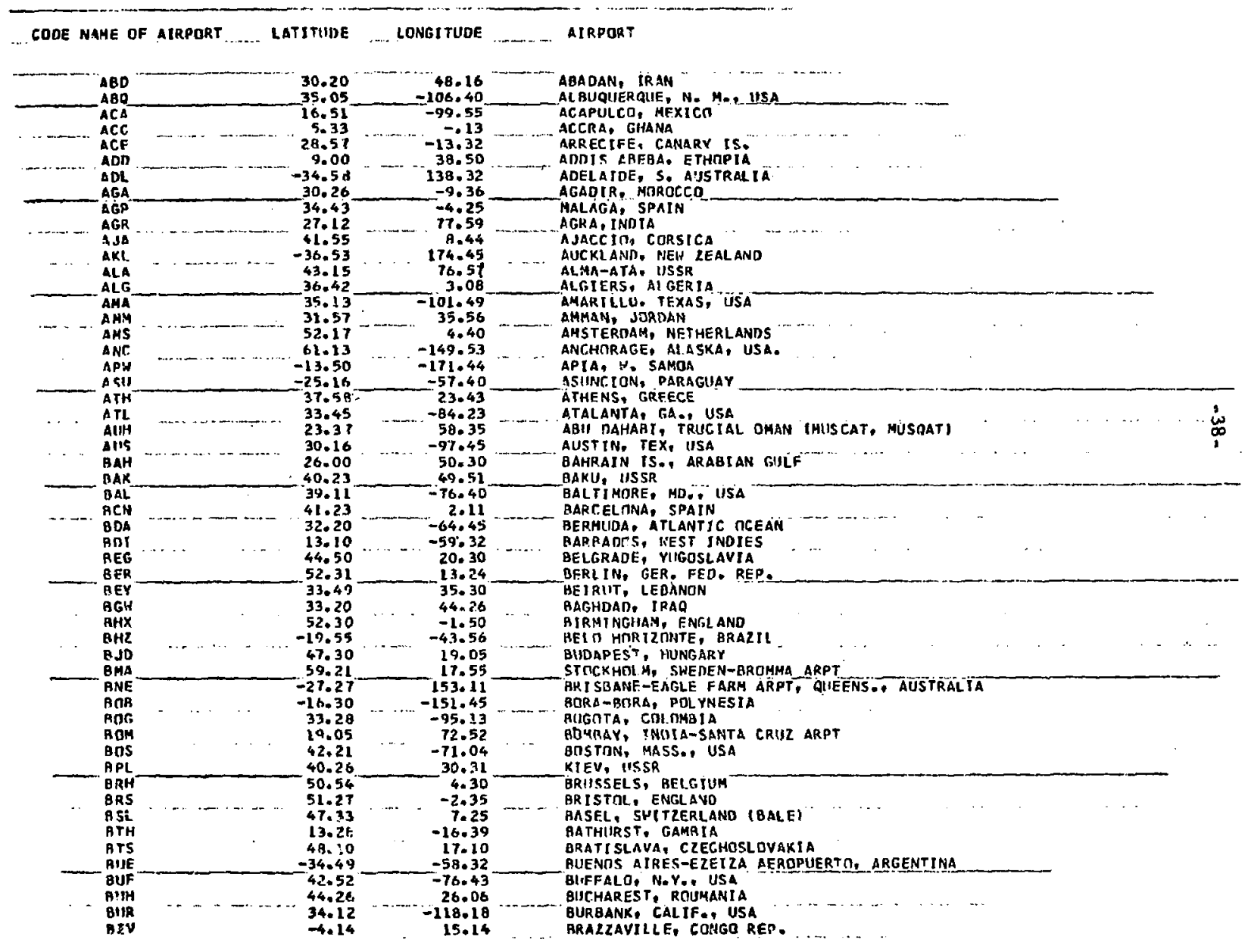




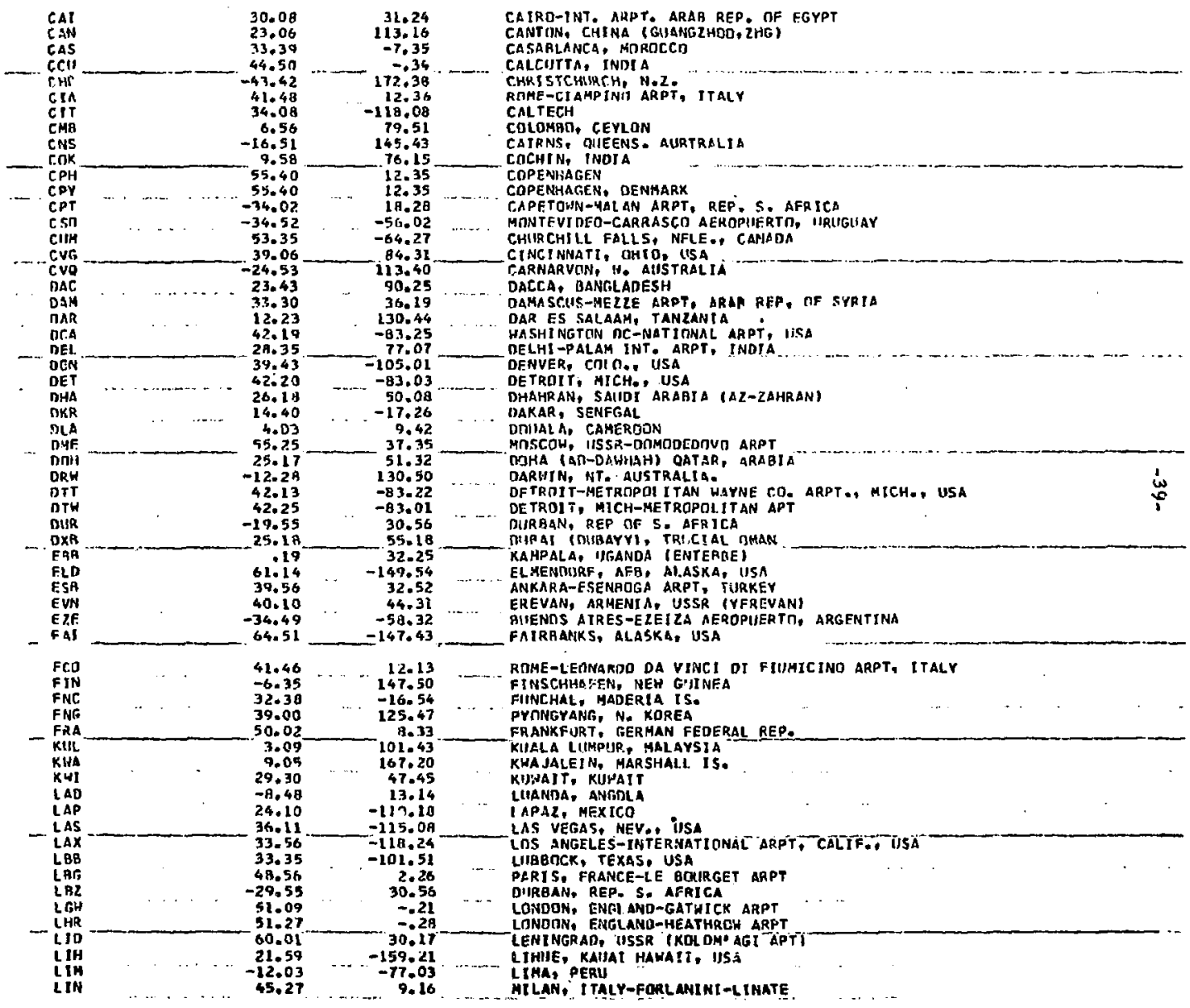




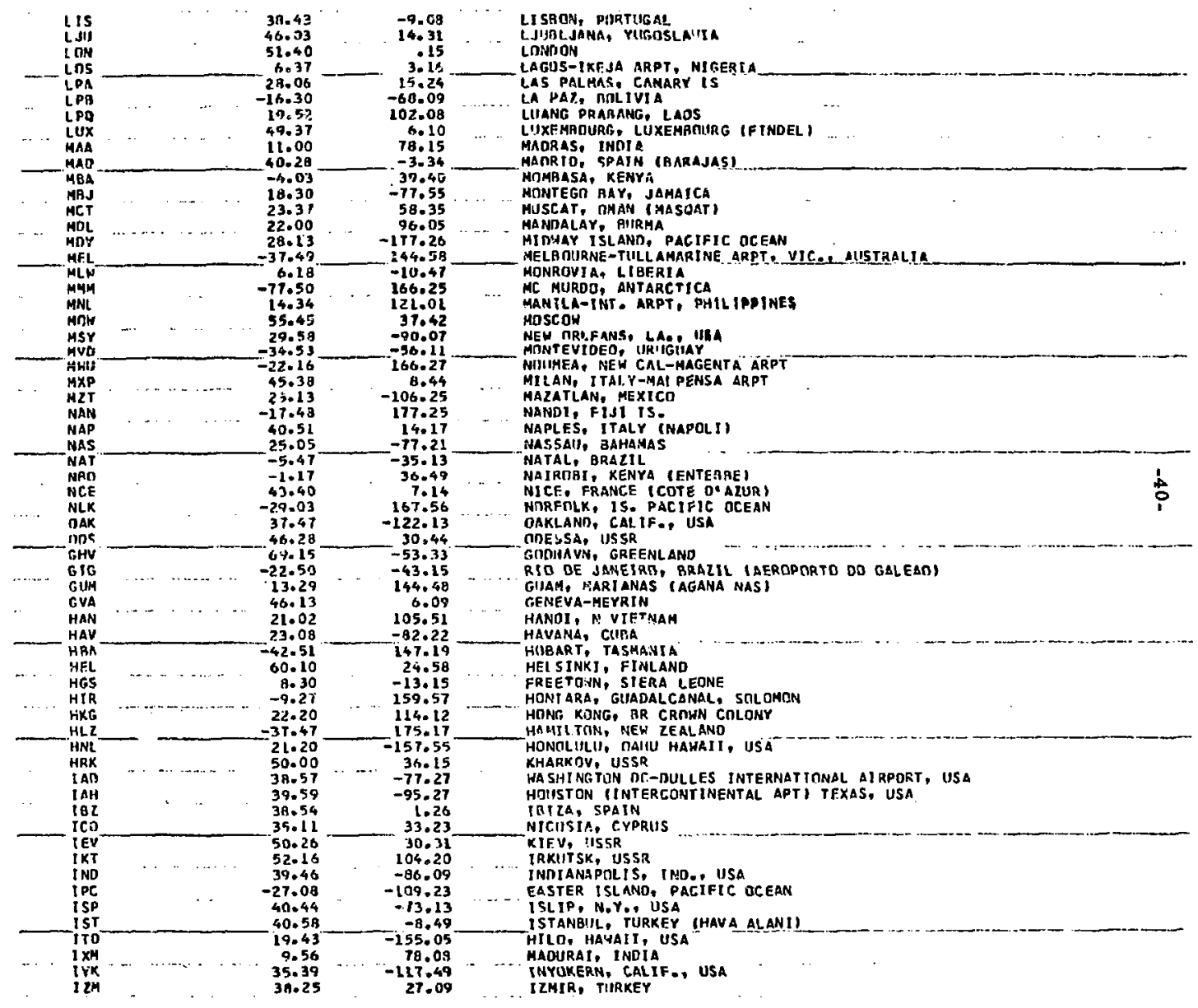




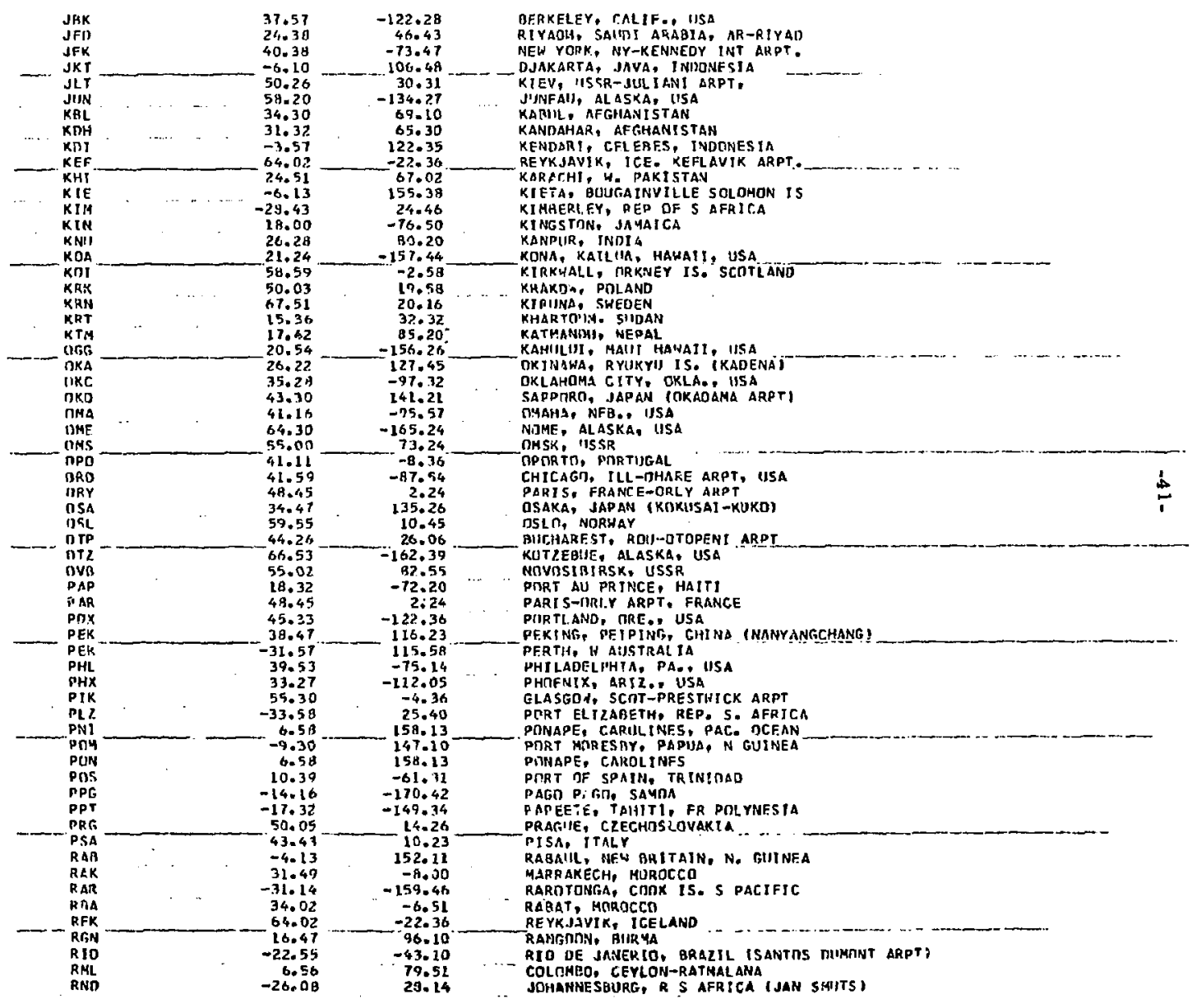




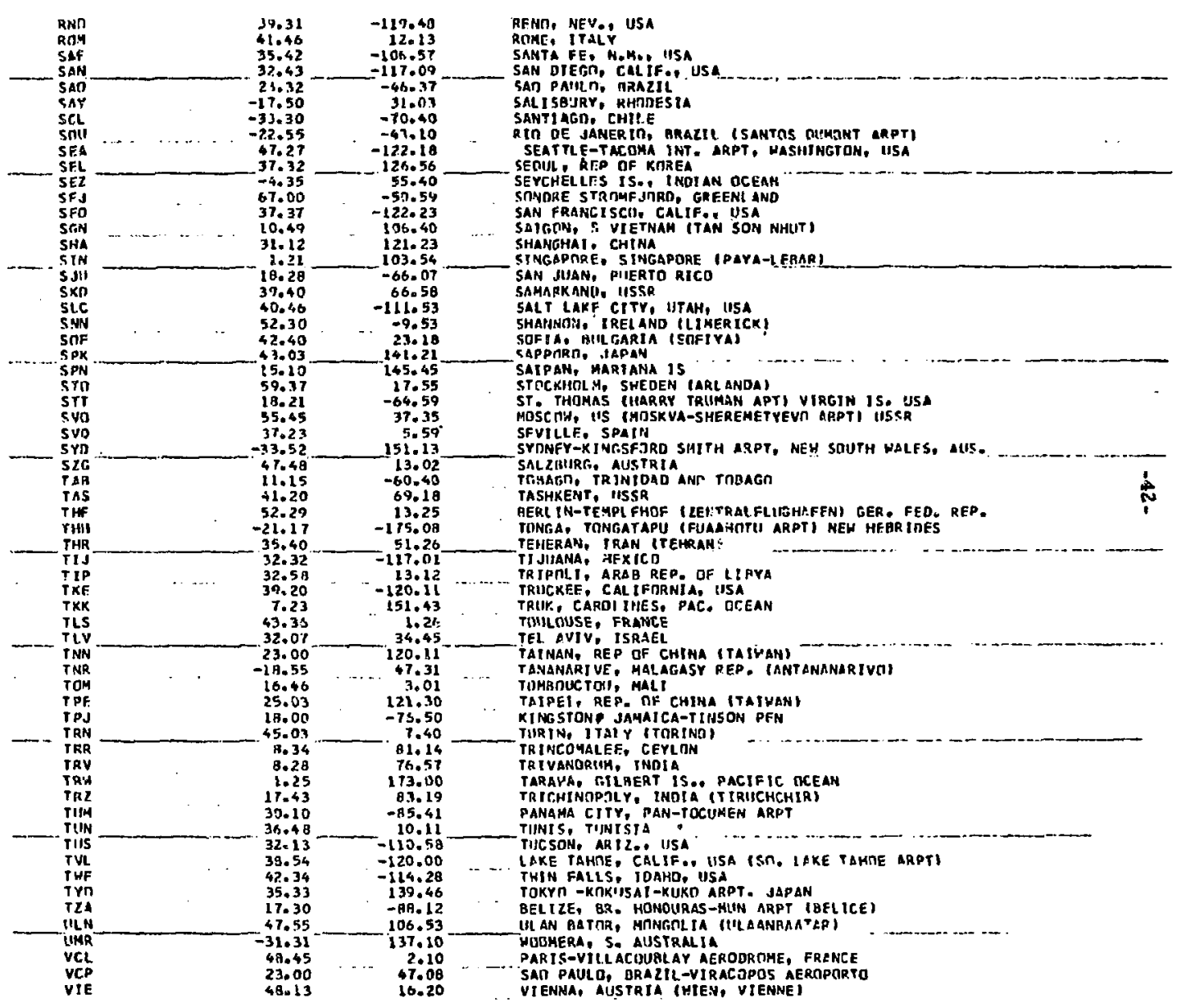




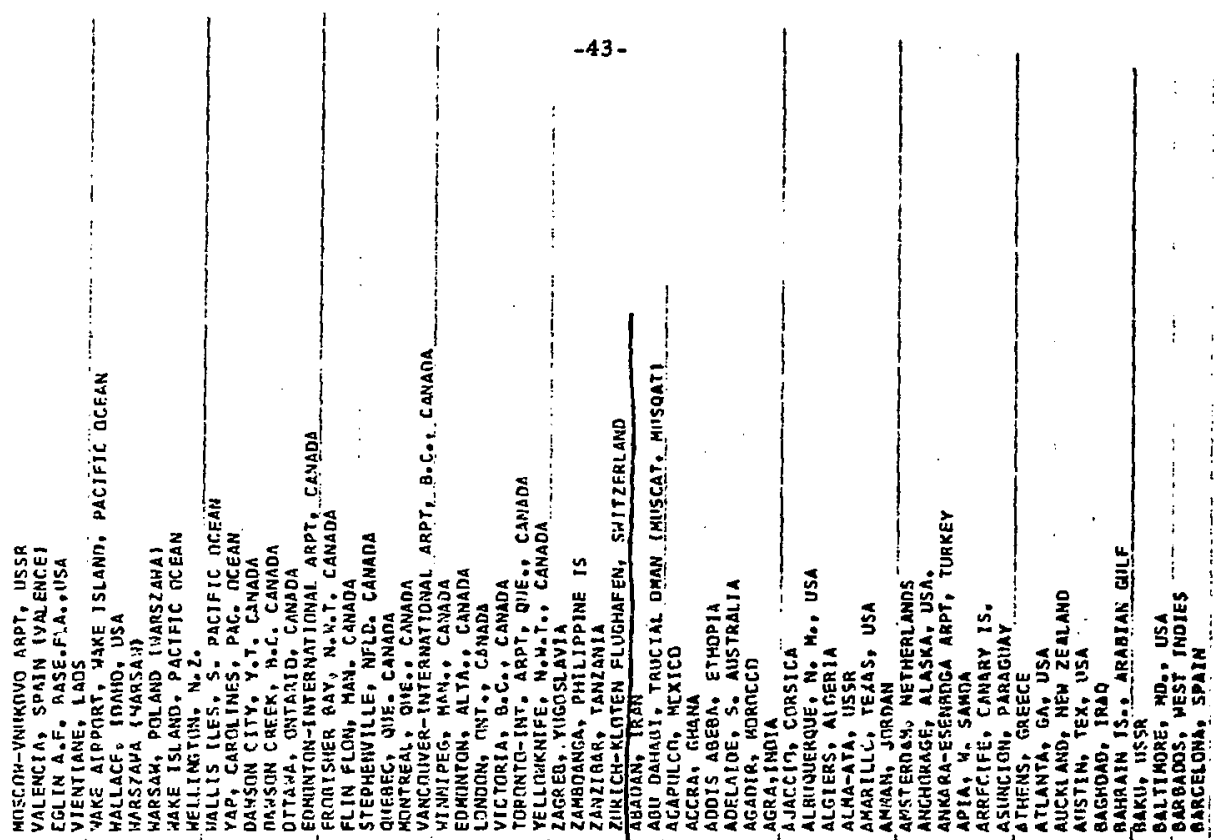

F m

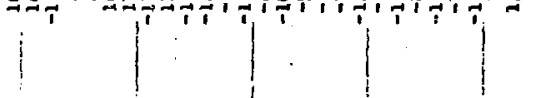

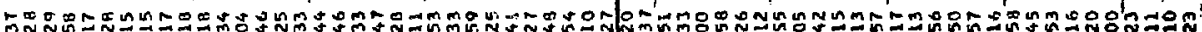

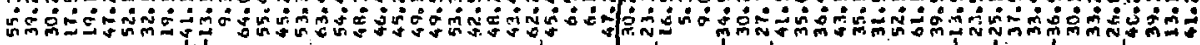

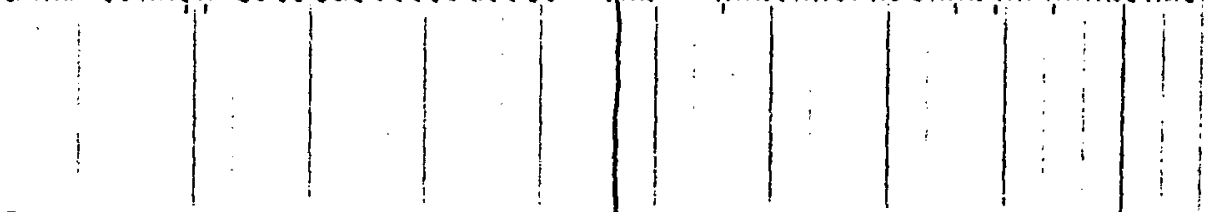

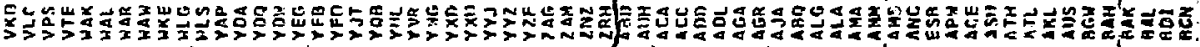




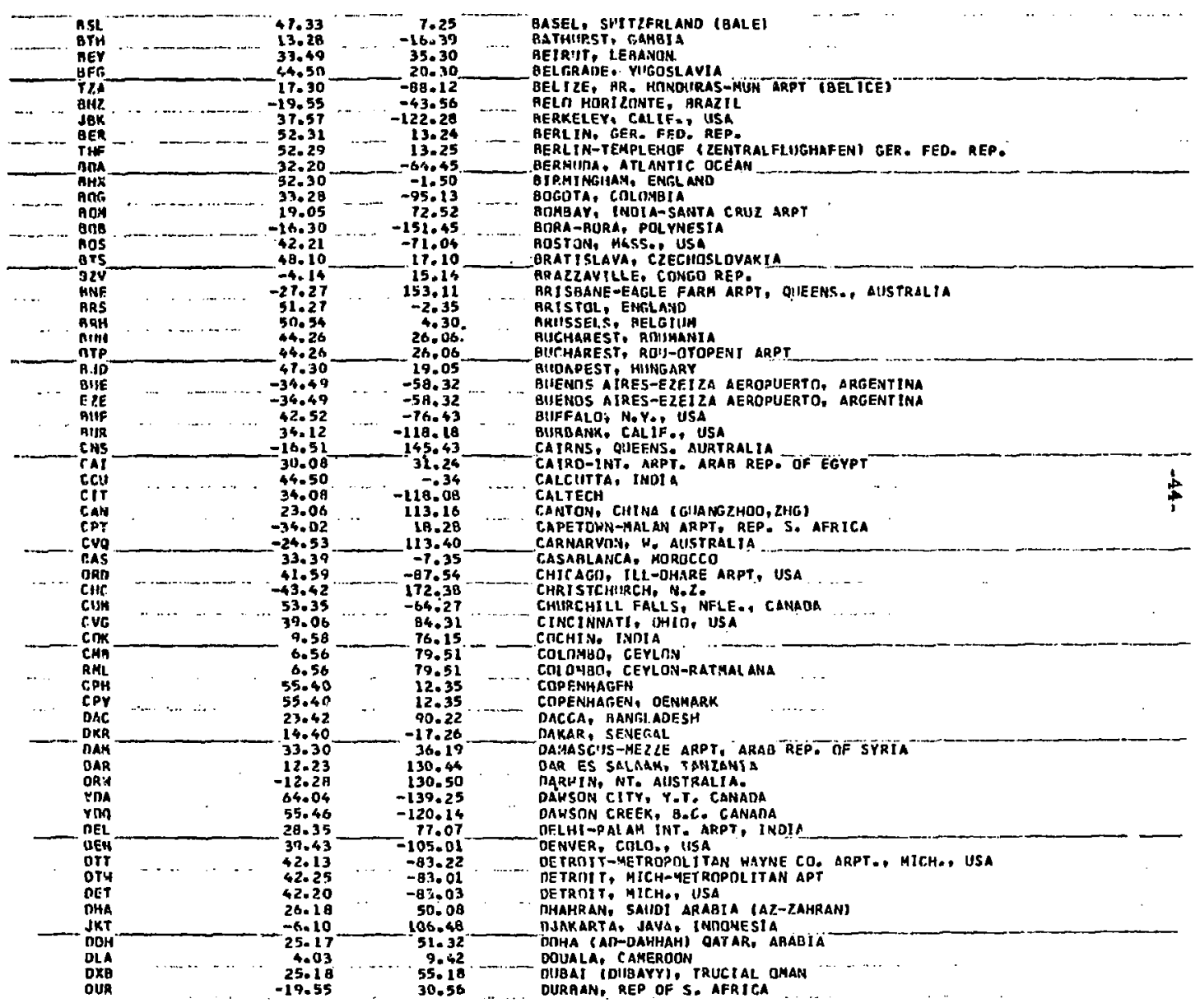



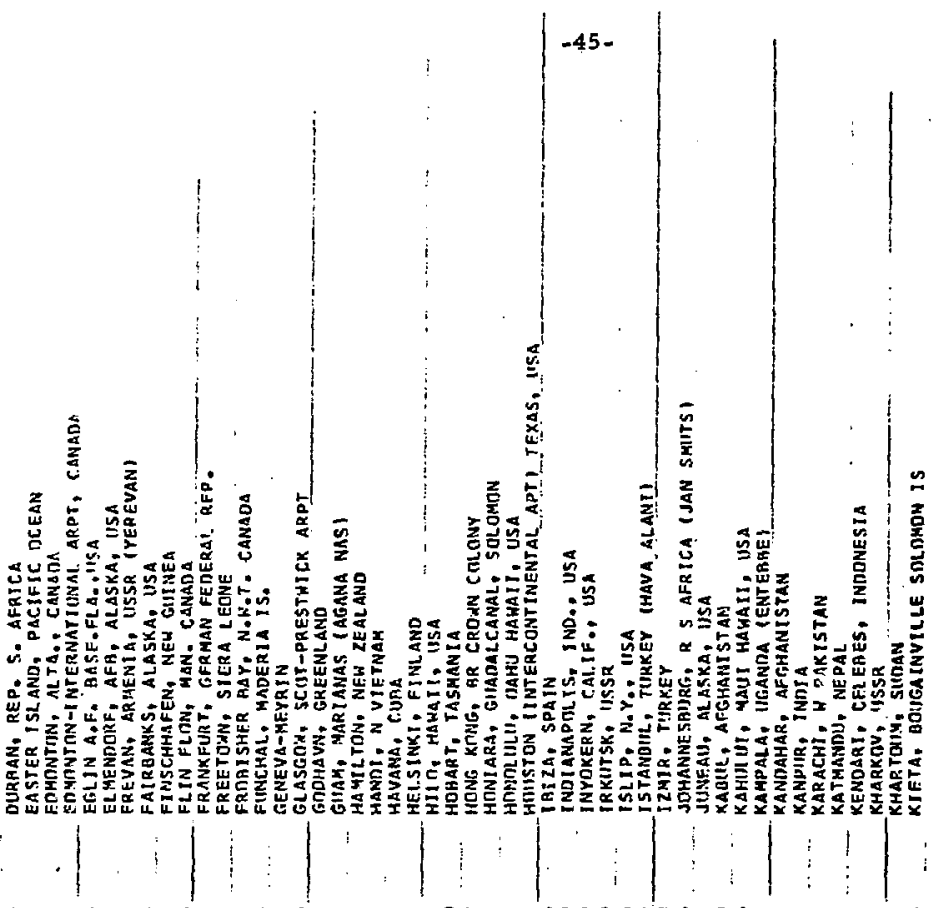

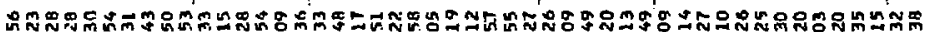

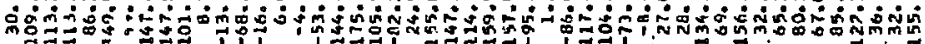
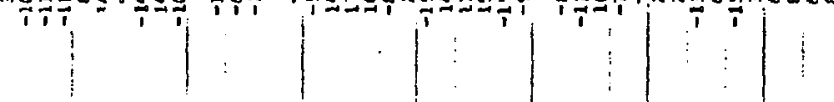

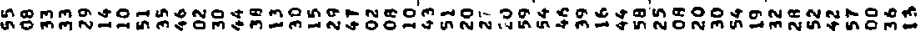

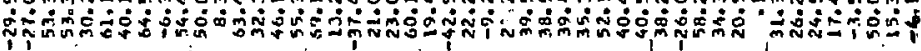
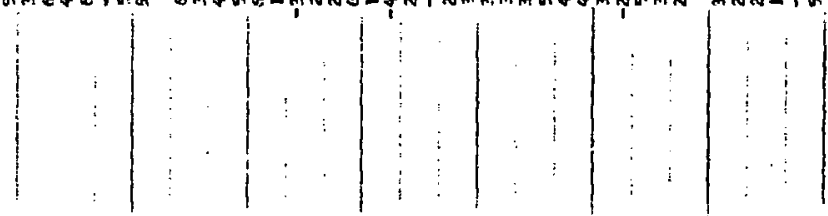

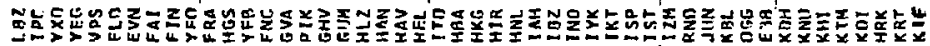
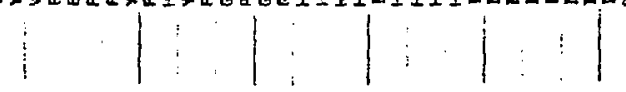


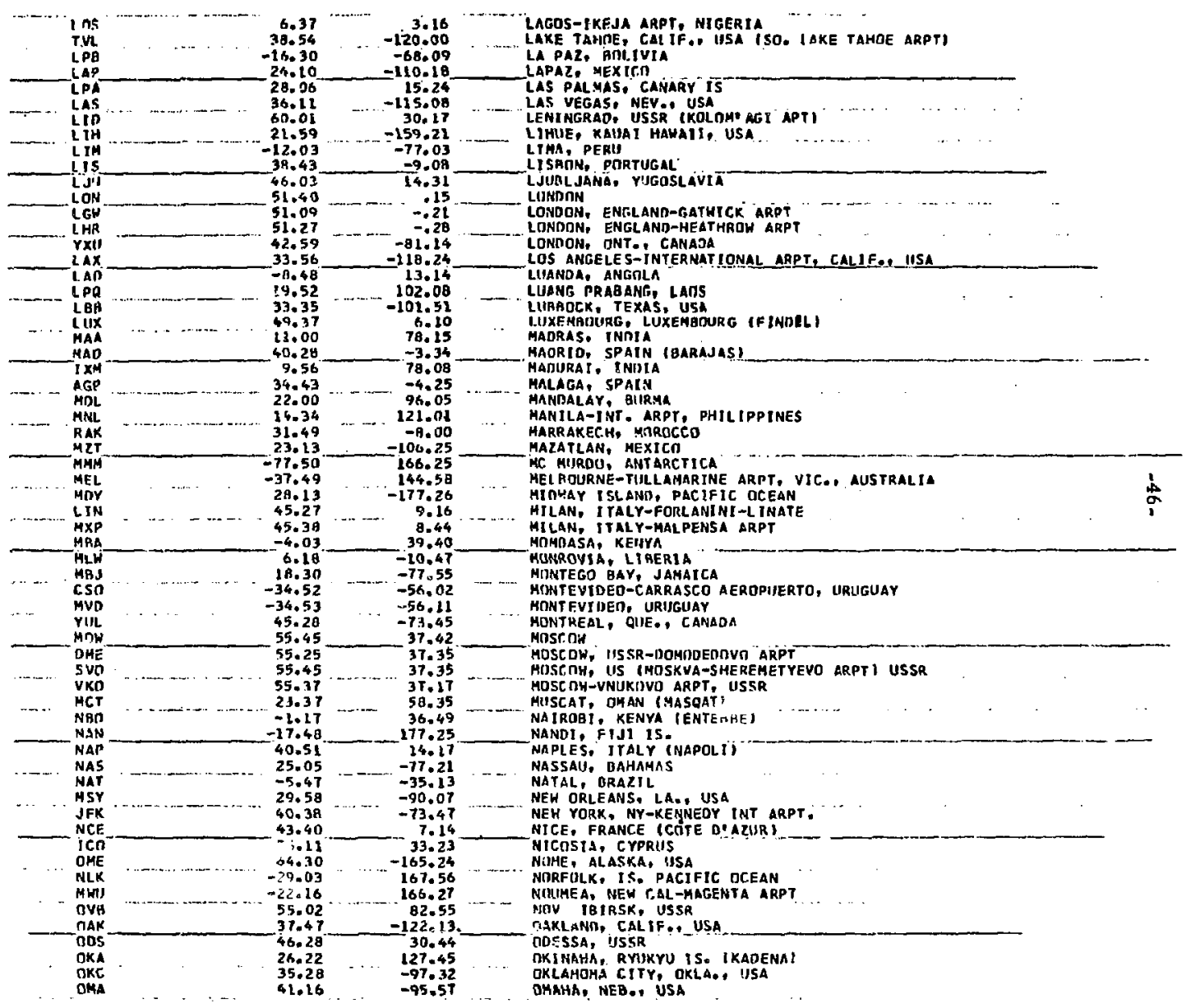




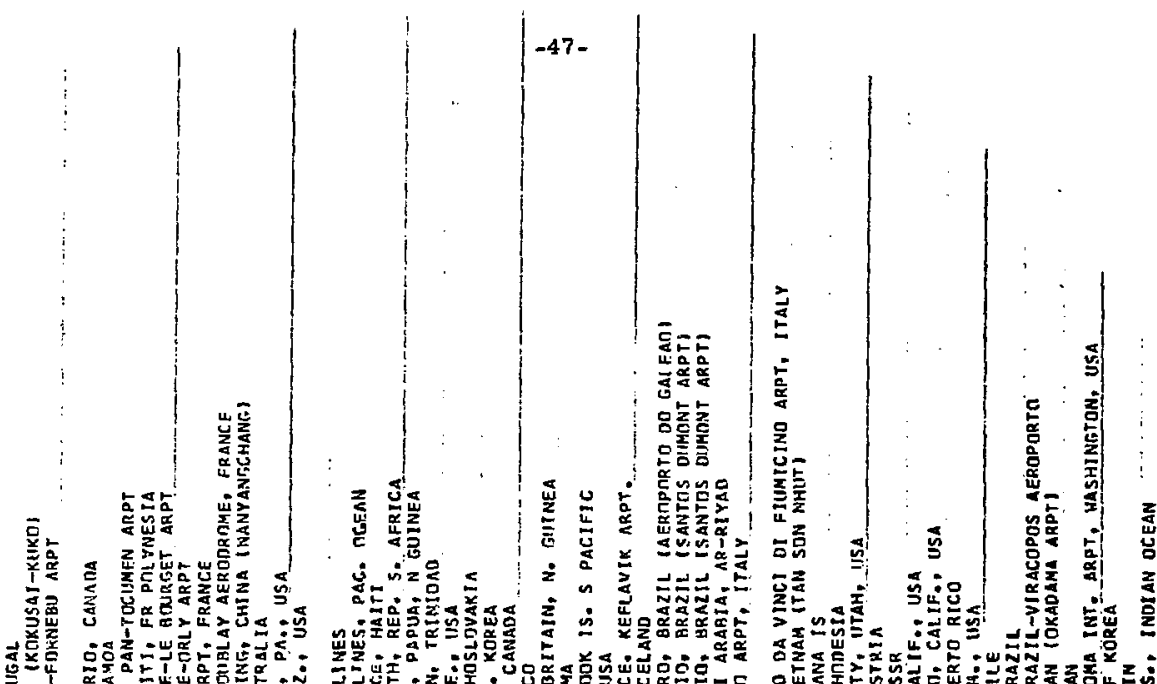

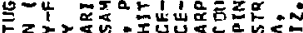

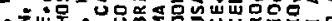

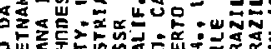

胥

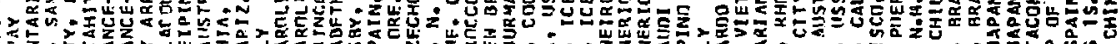

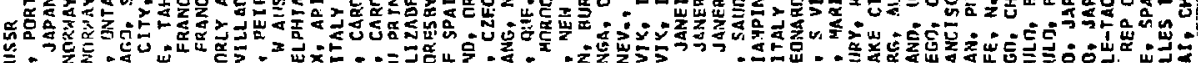

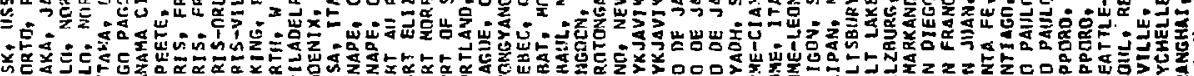

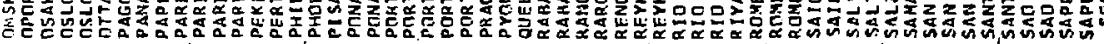
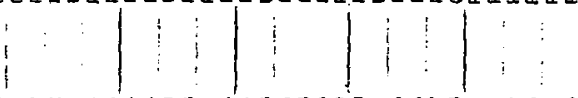

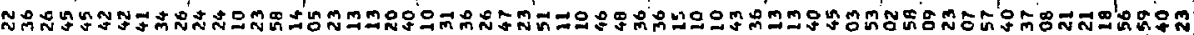

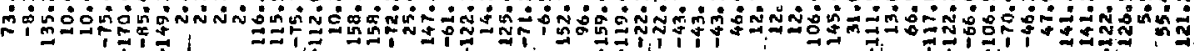

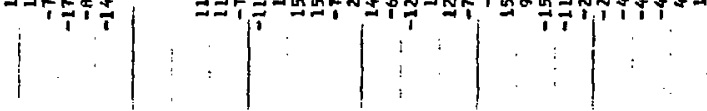

足二舟出出

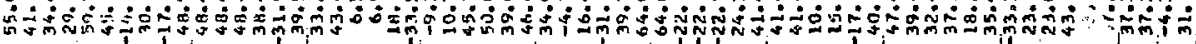
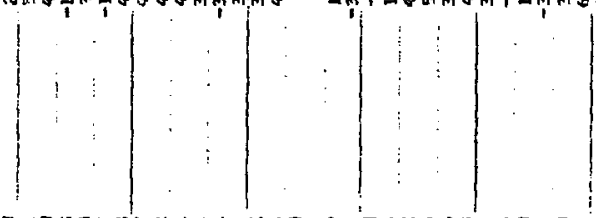

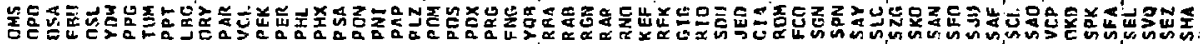


AT MACH 1.2

SCHEQULE BLOCK TIME ON ONE WAY(HRS) = G.T

RATE OFI CLIMB (FEET/MIN) 3333.333

CRUISINB ALTITUDE (FEET) : ¿A0̈000 - RATE OF DESCENT(FEET/MIN) 3333.333

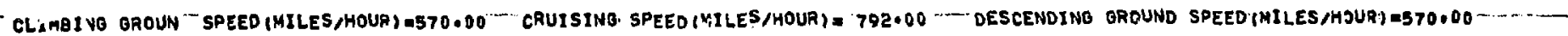
FLIGHT ALTITUDE ANO DÏSTANCE: PROFILË IN $1 / 10$ HOUR INCREHENTS.

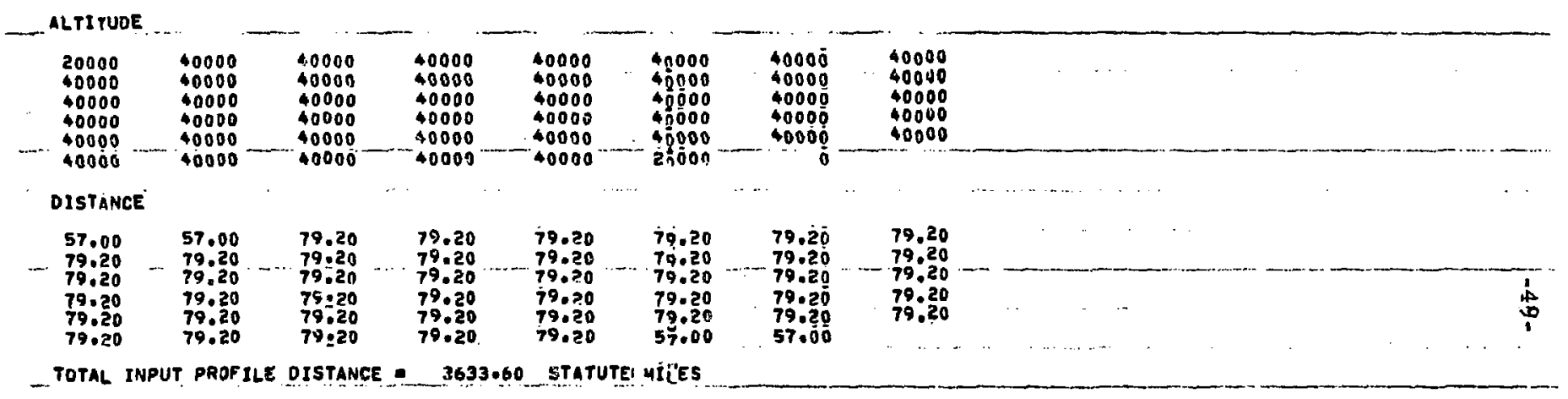

\section{FLIGHT PROFILE TIME $\quad 4 \cdot 70$ HOUGS IN TḦE AIR}




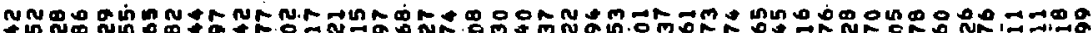

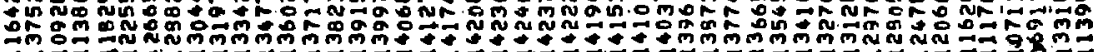

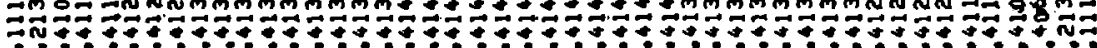
(1)

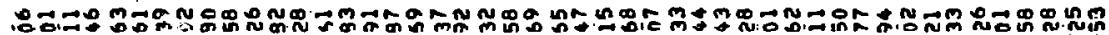

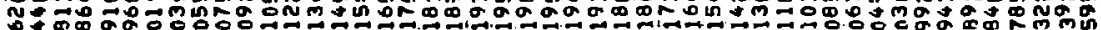

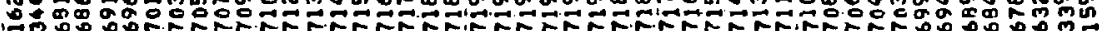

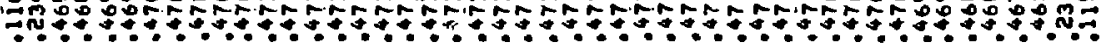
1
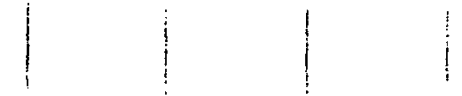

0 0.OOHNNOR

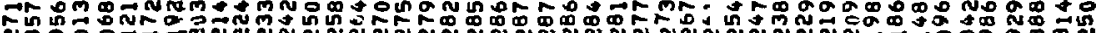

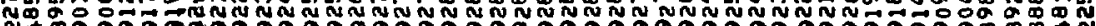
NT

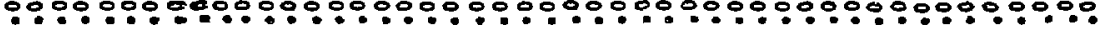

4 a n uno

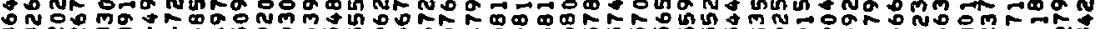

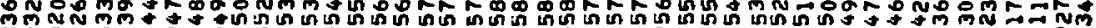

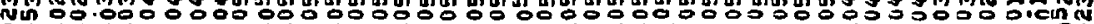

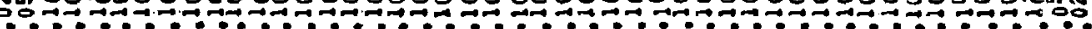

\section{-} (1)
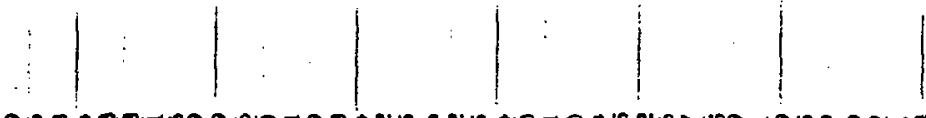

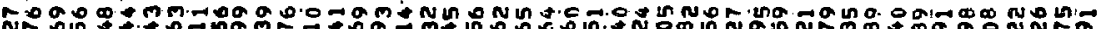

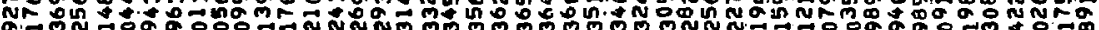

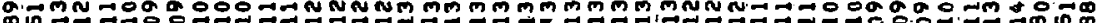

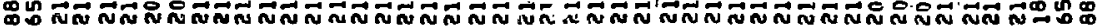
앴

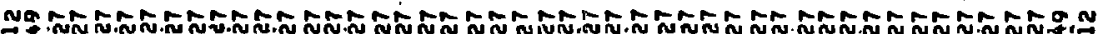

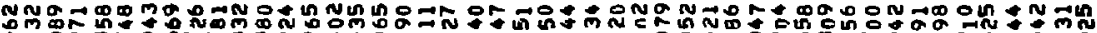
in $\Rightarrow 0$ \%

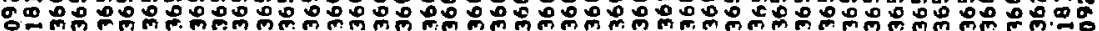

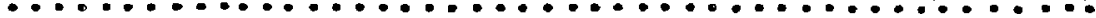
$\because:$ :

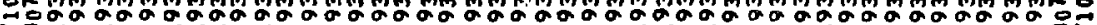
m

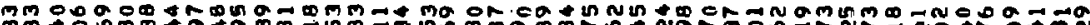
m. : inm tundor un un un un un unis un un un un un

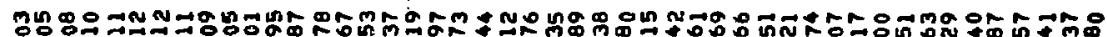

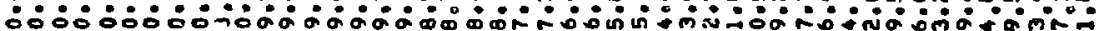

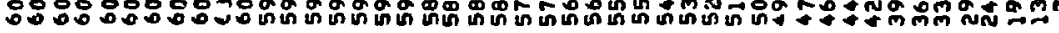

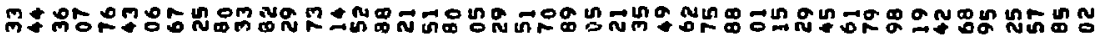

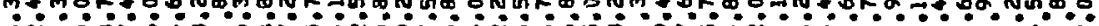

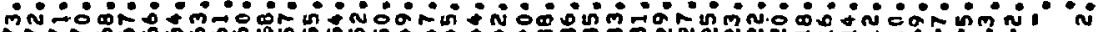

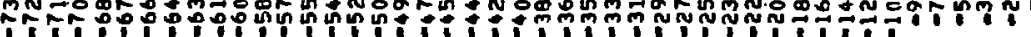

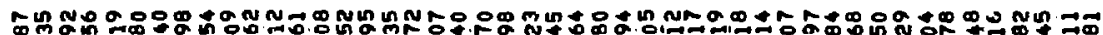

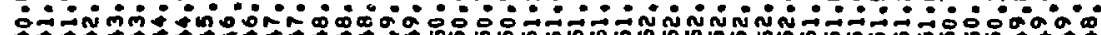

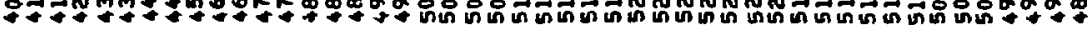




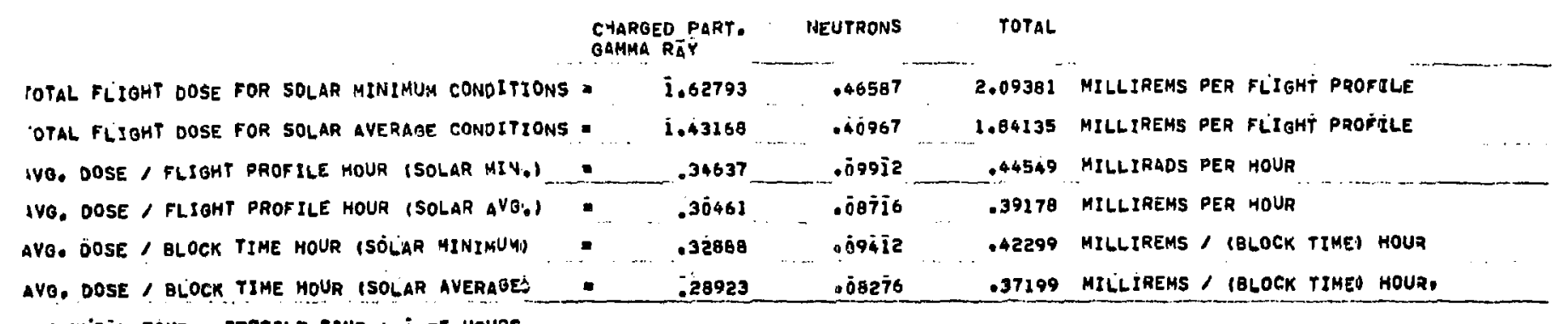
.EO BLDĆK TIME - PROP ILE TIME * D.25 HOURS 


\section{APPENDIX D}

WEIGHWEI AVERAGES AND STATISTICAL ERRCRS

1. NORMALLY DISTRIBUTED VARIABLES

In computing the sample mean of some function $F(A)$ of the kinematical variables $A$ of the decay, events with one pion in the $\omega$ mass region are given welght one and counted once while events with both pion triplets in the $W$ mass region are given weight one-half and counted as separate events.

$$
\begin{aligned}
\langle F(A)\rangle & =\sum_{1=1}^{N} w_{1} F\left(A_{1}\right) / \sum_{1=1}^{N^{\prime}} w_{1} \\
& =\frac{1}{N} \sum_{1=1}^{N !} w_{1} F\left(A_{1}\right)
\end{aligned}
$$

where $P\left(A_{i}\right)$ is any function of the kinematical variables $A_{1}$ of the 1 'th event, $w_{1}=1$ or $1 / 2$ is the velght function, $N$ is the number of events with at least one pion triplet in the $\omega$ mass region, and $\mathbb{N}^{\prime}=N+$ the number of events $w^{\prime}$ th both pion triplets in the $W$ mass region.

The statistical errors are approximated by the expression

$$
\begin{aligned}
& \sigma^{2}[F(A)]=\frac{1}{n^{2}}\left[\sum_{i=1}^{N^{\prime}} w_{1}^{2}\right] \\
& x\left[\frac{1}{-1} \sum_{1=1}^{N} w_{1} F^{2}\left(A_{1}\right)-\left(\frac{1}{N} \sum_{1=1}^{N} w_{1} F\left(A_{1}\right)\right)^{2}\right]
\end{aligned}
$$

2. NON-TORNALLY DL:TTIT:UTED VARIABLES

The variables $R_{M}^{2}$ and $J(J+1)$ elver by Eqns. $(C-7)$ and $(B-5)$ are not normally distributed. Hovever, for each hypothesis of such varlables one can calculate a chi-square and different hypotheses can be compared using a $11 \mathrm{kl}$ lhood ratic or confidence level. Suppose one has $n$ equations of the form

$$
R=\left\langle F_{k}\right\rangle /\left\langle G_{k}\right\rangle
$$

for $k=1,2, \ldots, n$ where $\left\langle F_{k}\right\rangle$ and $\left\langle G_{k}\right\rangle$ are expectation values of n functions of the kinematical variables $A$ which are approximated by

$$
\left\langle F_{k}\right\rangle=\sum_{i=1}^{I^{\prime}} w_{1} F_{k}\left(A_{i}\right) / \sum_{1=1}^{n^{\prime}} w_{1}
$$

and

$$
\begin{aligned}
= & \frac{1}{N} \sum_{i=1}^{N} v_{i} F_{k}\left(A_{i}\right) \\
\left\langle G_{k}\right) & =\frac{1}{N} \sum_{i=1}^{\mathbb{N}} w_{1} G_{k}\left(A_{1}\right)
\end{aligned}
$$

where $x_{1}=1$ or $1 / 218$ the velght function, $N$ is the number of events with at least one pion triplet in the $\omega$ mass region, and $N^{+}=\pi+$ the number of events with both pion triplets in the $W$ mass regtion.

The equations to be satisfied by the parameter can be written 
COMPUTED GREAT CIRCLE LEO DISTANCE $=\quad 56+9.9$ THE INITIAL COURSE ANGLE $=35.038$

LEG START LATITUDE * 34.00 LEG STAAT LONGITUDE: -118.15

LEG END LATITUDE. = 48.72 LEG ENO LONGTTJUE = 2.38

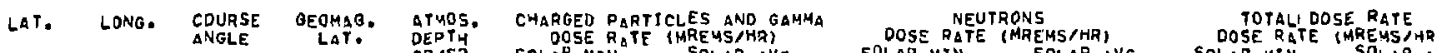

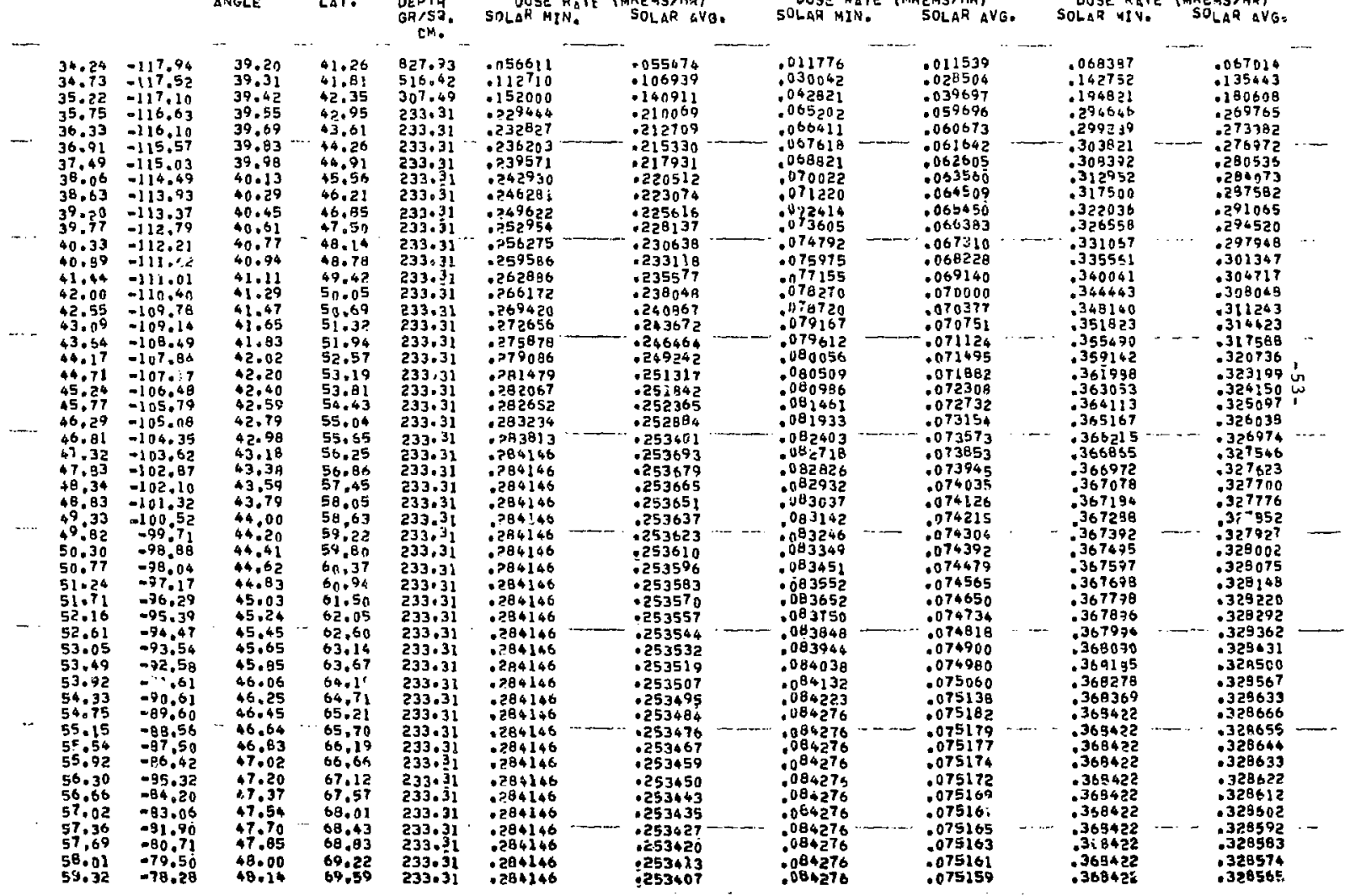




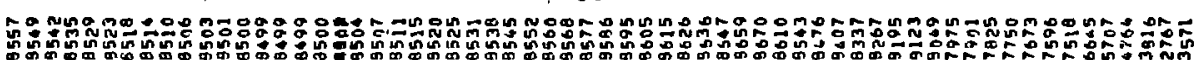

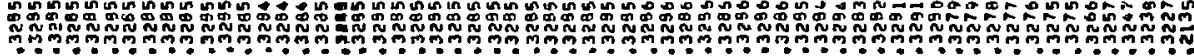

WN

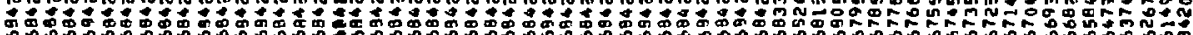

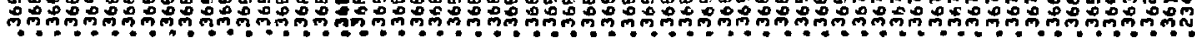

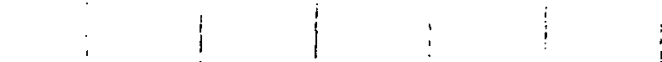

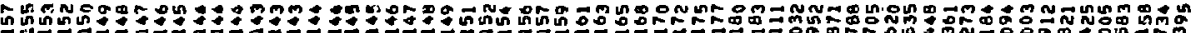

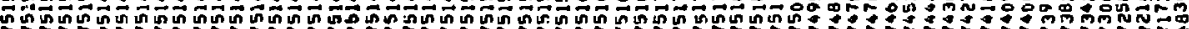

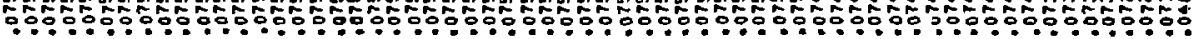
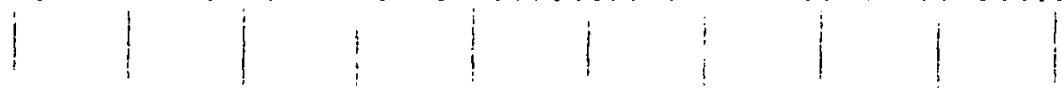

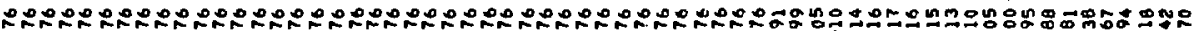

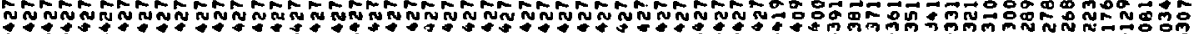

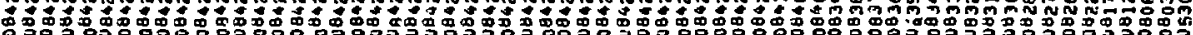

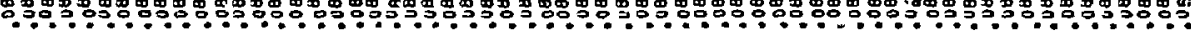

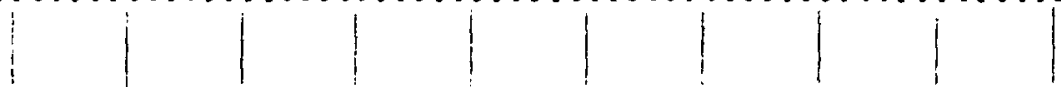

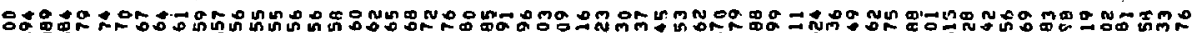

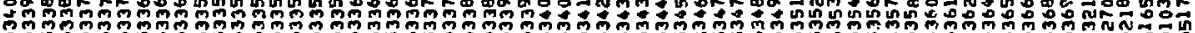

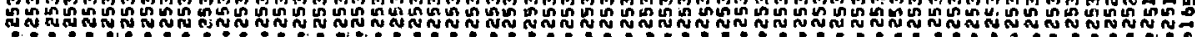

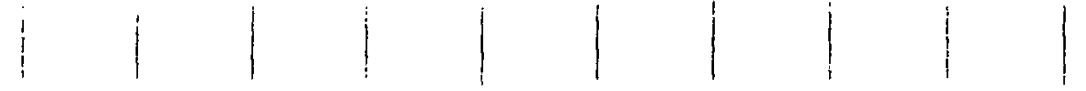

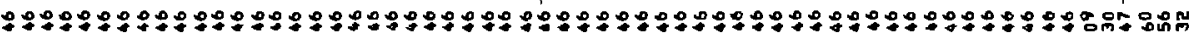

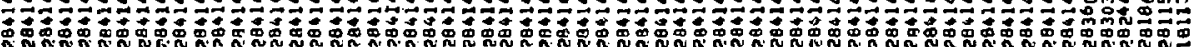

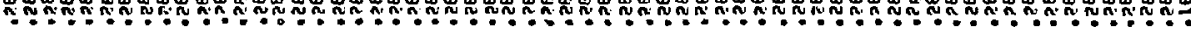

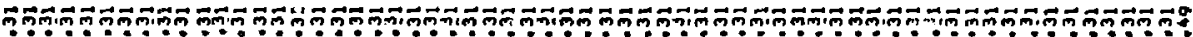

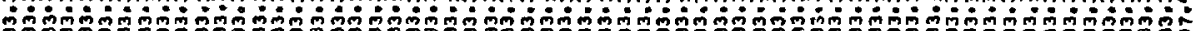

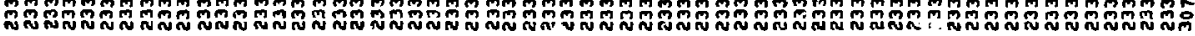

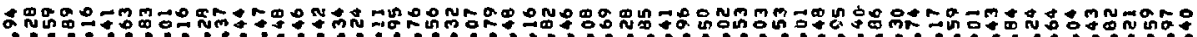

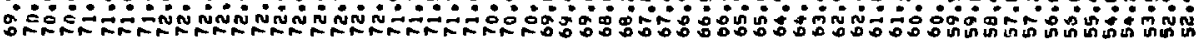

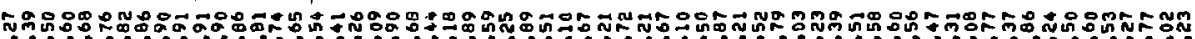

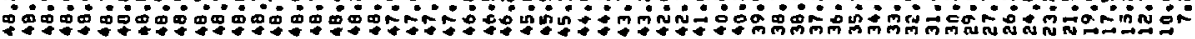

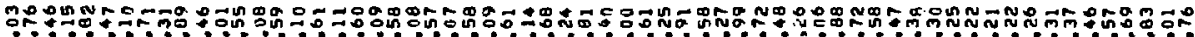

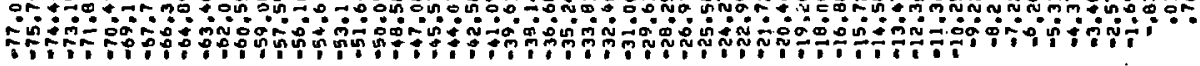

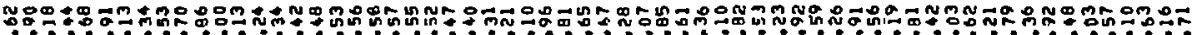

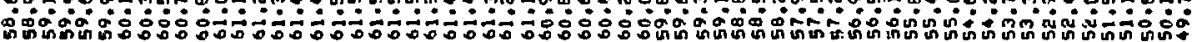


$-55-$

용

N

풍

$\because$.

m

踊

:응

羽ำ

영

용.

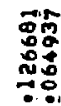

$\overrightarrow{50}$

ปี้อ

$\Rightarrow$ ?

No

$\because 0$

ถีก

嘼吕

जี่์

$+8$

$\div \div$

웅

: 


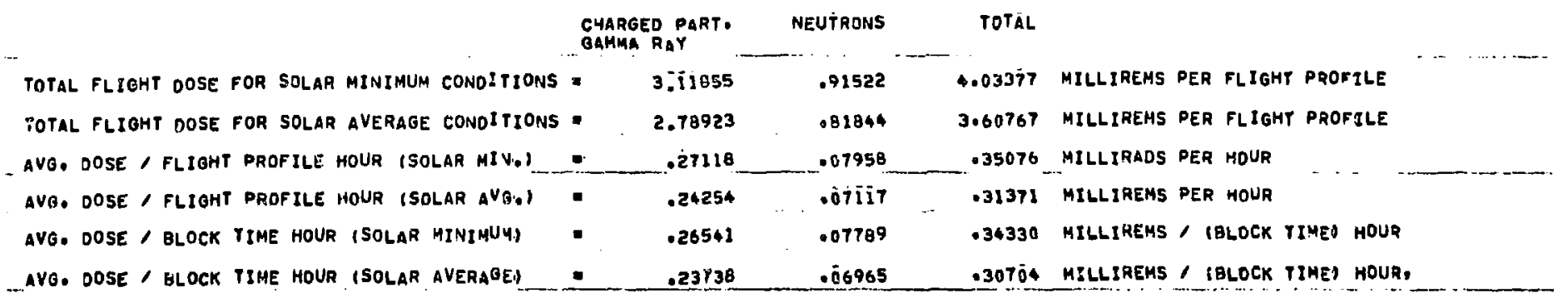
LES OLOCK TIME = PROFILE TIME - 0.25 HOURS 

FLIGHT ALTITUDE AND DÍSTANCE PROFILE IN $1 / 10$ HOUR INCREMENTS,

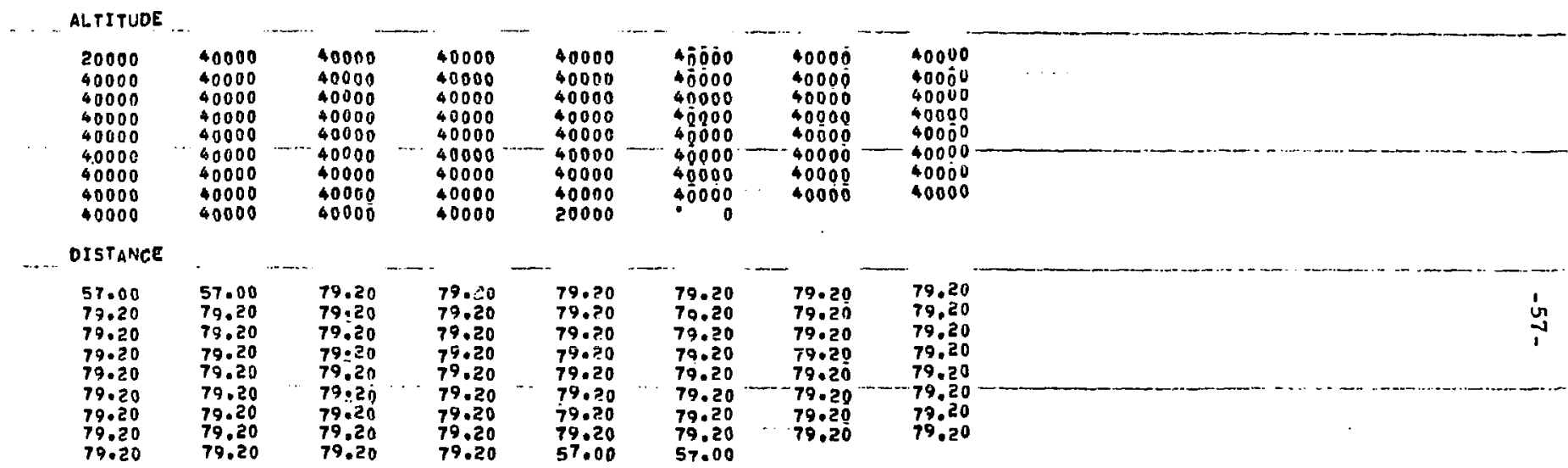

TOTAL INPUT PROF ILE OISTANCE = $5455 \cdot 20$ STATUTE YIf́ES 
COMDUTED GREAT CIRCLE LEG OISTGNCE = 5450.5 TḦE INITIAL COUASE ANGLE - 3I1.947

LEB STAR: LATITUOE - 91.40 LEg staAt LOMBITUDE - .15

- LES END LAT̈1TUdE. -34.00 TEO ENg LONOLTUOE - -118.25

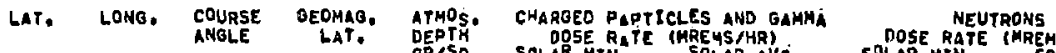

NEUTRONS TOTAL DOSE RATE

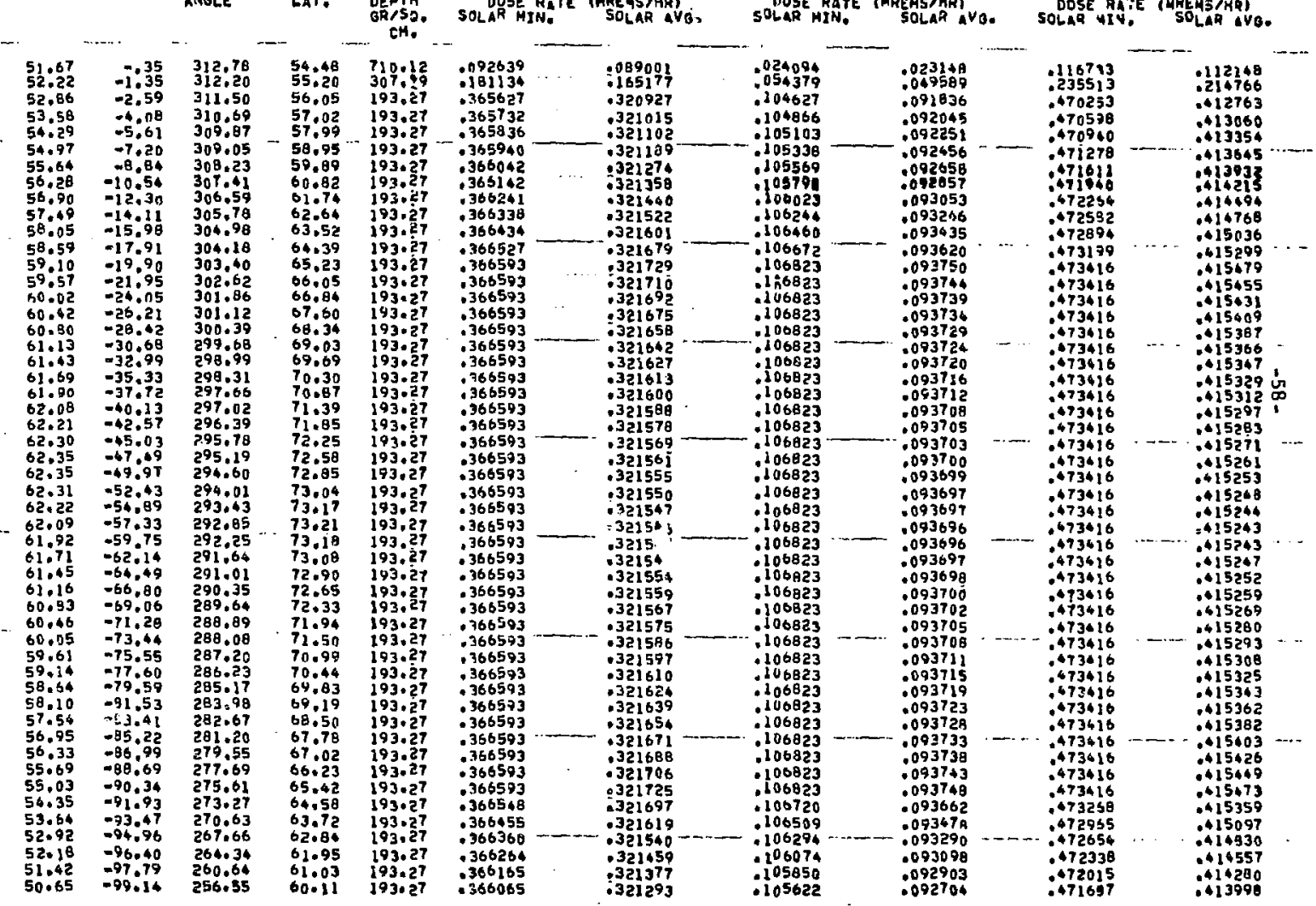




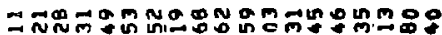

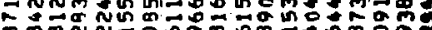

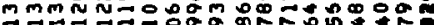

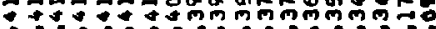

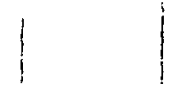

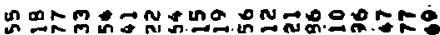

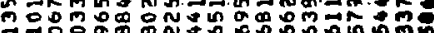

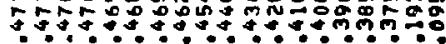

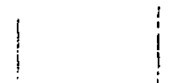

mamo

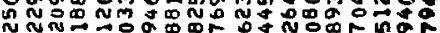
N $N \overrightarrow{0} \rightarrow 0$ O

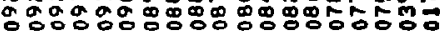

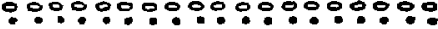

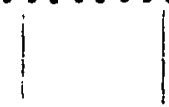

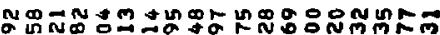

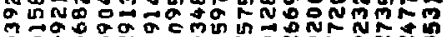

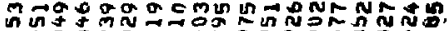
乌乌

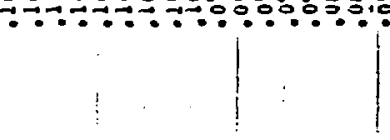

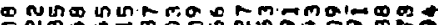

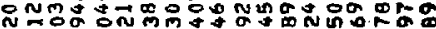

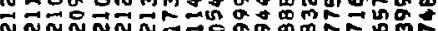

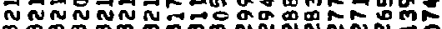

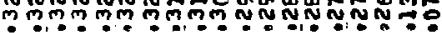

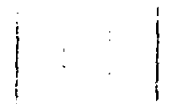

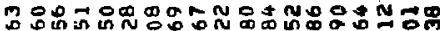
o mo

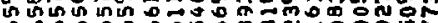
m

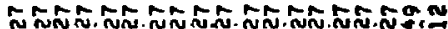
- - - - - - - - . mmmmm

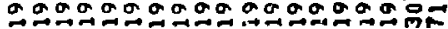

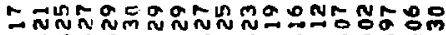

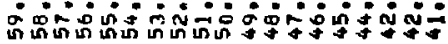

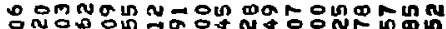

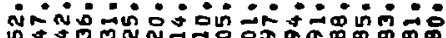

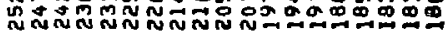

- on 0 t m mar mo on

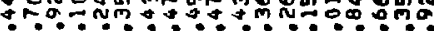

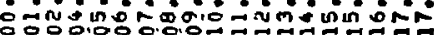
문

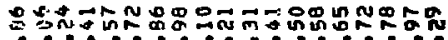

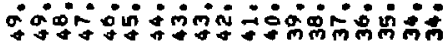




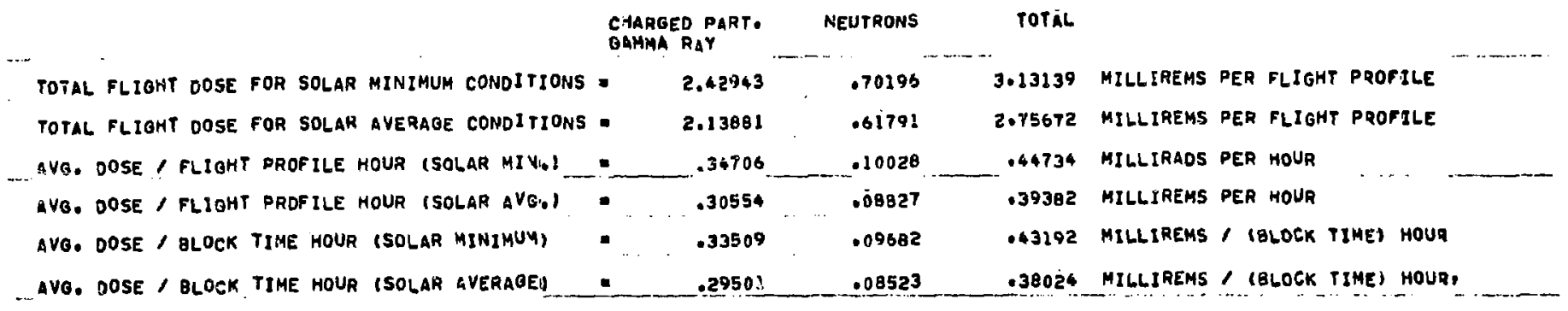
LEO BLOCK TIME a PROFILE TIME - 0.25 HOURS 
SCHEDULE RLOCK TIME ON ONE WAY (HRS) = 5.1

RATE OF CLIME (FEET/MIN) - 2000.000

CLIMEIVO GROUN SPEEOPHILES/HQUAI $\times 413.00$
CRUTSINB ALTITUDE (FEET) $\quad 36000$ RATE DF DESCENT (FEET/MIN) 20000000

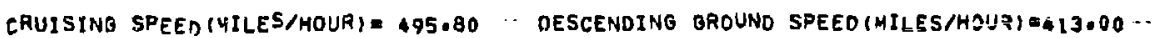

FLIGHT ALTITUDE ANO DISTAVCE PROFILE IN $1 / 10$ hUUR INCREMENTS.

ALTITUDE

\section{0}

36000

36000

36000

35000

36000

24000

36000

36000

36000

36000

36000

12000

36000
36000
3600
3600
3600

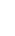

DIST ANCE

$\begin{array}{lllllllll}41.30 & 41.30 & 41.30 & 49.58 & 49.58 & 49.58 & 49.58 & 49.58 \\ 49.58 & 49.58 & \ldots & 49.58 & 49.58 & 49.58 & 49.58 & 49.58 & 49.58 \\ 49.59 & 49.58 & 49.58 & 49.58 & 49.58 & 49.58 & 49.59 & 49.58 \\ 49.58 & 49.58 & 49.58 & 49.58 & 49.58 & 49.58 & 49.58 & 49.58 \\ 49.58 & 49.58 & 49.58 & 49.58 & 49.58 & 49.58 & 49.58 & 49.58 \\ 49.58 & 49.58 & 49.58 & 49.58 & 49.58 & 49.58 & 49.58 & 49.58 \\ 41.30 & 41.30 & 41.30 & \ldots & & & 4.58\end{array}$

TOTAL INPUT PRDFILE DISTANCE - 2478.90 STATUTE MIIES

FLIGHT PROFILE TIHE

- 5.10 hoURs In THE aIR 
COMDUTED GREAT CIRCLE LEG DISTANEE = 2453.1 THE INITIAL COURSE ANGLE * 65.982

LEQ START LATITUDE - 34.00 LEO STAPT LONGLTUDE = $=118.15$

LEO EÑO LATITUDE1 = 40.63 LEO ENDI LONGITUDE = -73.77

LAT. LONG. COLRSE GEOMAG. ATHOS. CHARGEO PIRTICLES AND GAMHA TOTALI DOSE RATE

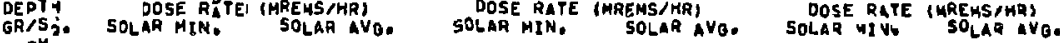

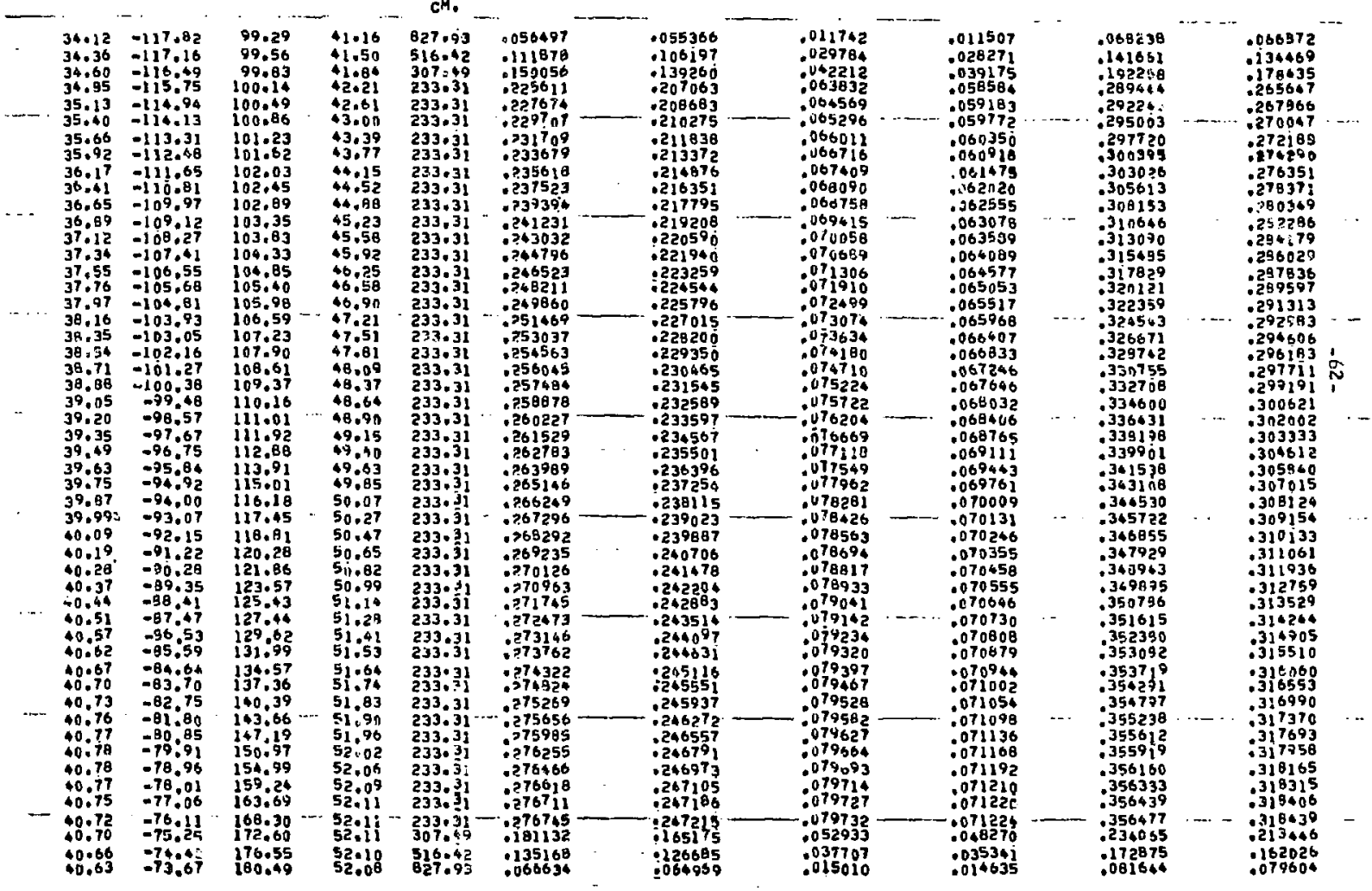


TOTAL FLIGHT DOSE FOR SOLAR MINIMUM CONDITIONS

TOTAL FLIGHT DOSE FOR SOLAR aVERAGE CONOITIONS

1.23432

.35626

1.59058

MILLIFEMS PER FLIGHT PQOFILE

AVO. DOSE / FLIGHT PROFILE HOUR (SOLAR MIV,

1.11310

.32116

1.43426

MILLIREHS PER FLIGHT PROFILE

AVO. DOSE / FLIGHT PROFILE hOUR (SOLAR aVa.) .24202

AYO. DOSE / BLOCK TIME MOUR (SOLAR MINIMUN) .21825 .06985

AVO DOSE OLOCK TEHE HOUR ISOLAR AVERAGEI

.23071

.06297 .31288

.28123

MILLIRAOS PER HOUR

.23071

.06659

-29730 MILLJREMS / (BLOCK TIME) HOUR LEO BLOCK TIME - PROFILE TIHE $\bullet 0.25$ HOURS 
RATE OF CLIHB (FEET/MIN) 3333.333

SCHEDULE BLOCK TIME ON ONE WAY(HRS)

- CLIMEIVE OROUN SPEED (HILES/HOUR) $=665.00^{-}$ CRUISING ALTITUDE(FEET)= 40000 RATE OF DESCENT(FEET/MIV) $=3333.333$

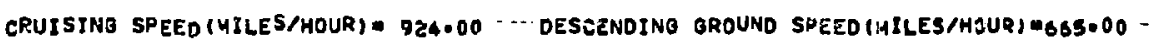

FLIOHT ALTITUDE AND DTSTANCE PROFILE IN I/10 HOUR INCREMENTS.

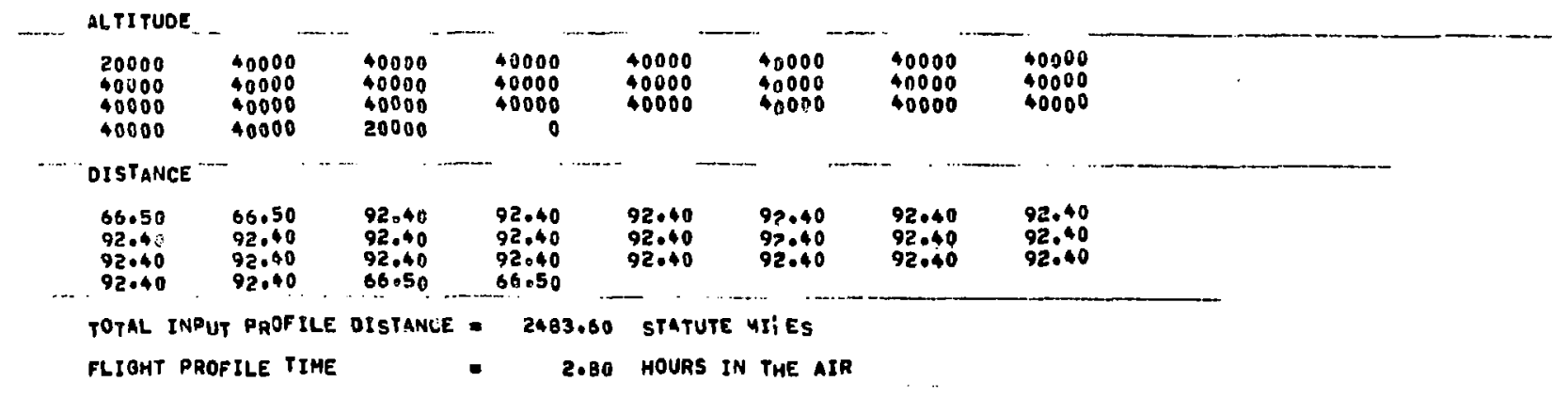


으은 n

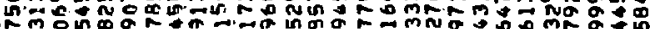

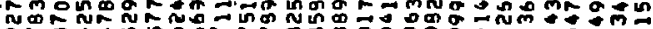

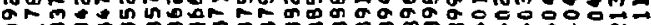

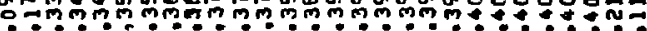

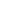

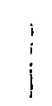

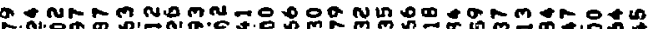
- $\rightarrow$ in ñoñ in

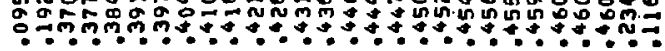<smiles></smiles>

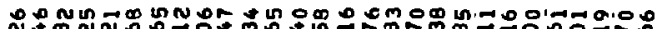

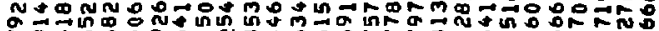

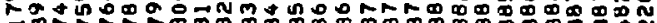

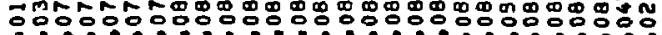

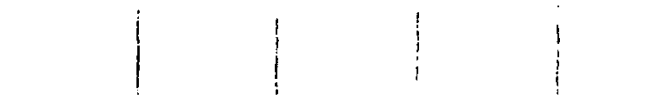

ODO*O ที่ง

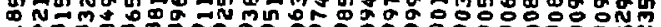

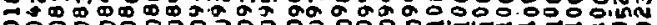

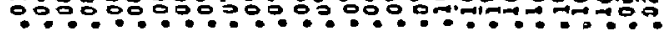
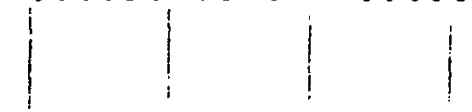

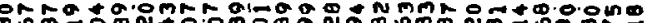

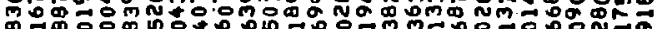

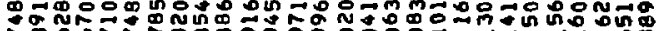
NMㅇㅅ

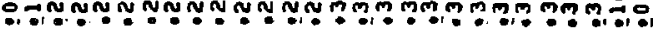
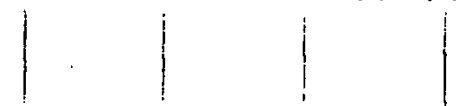

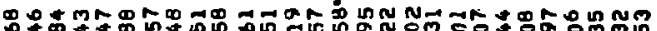

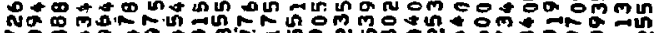

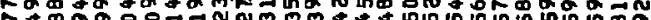

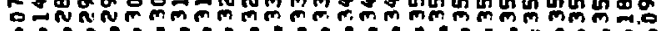

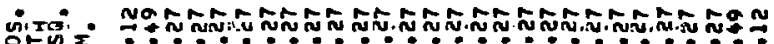

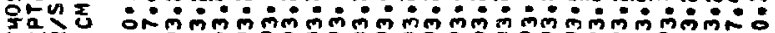

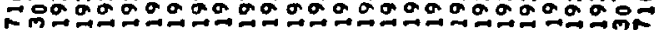

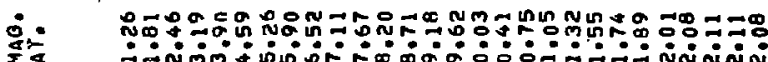

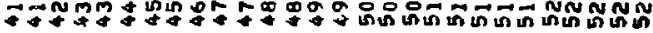

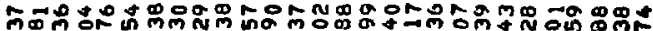
லே்

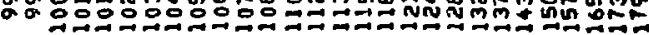

悹w i

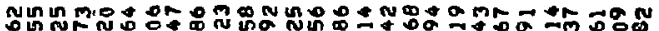
- 10. - roumneorutonogn山

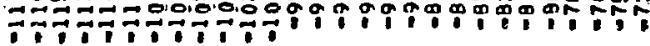

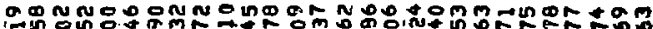

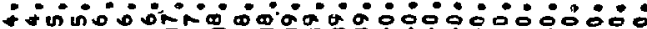

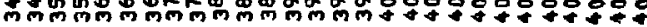


CHARGED PART. NEUTRONS TOTAL

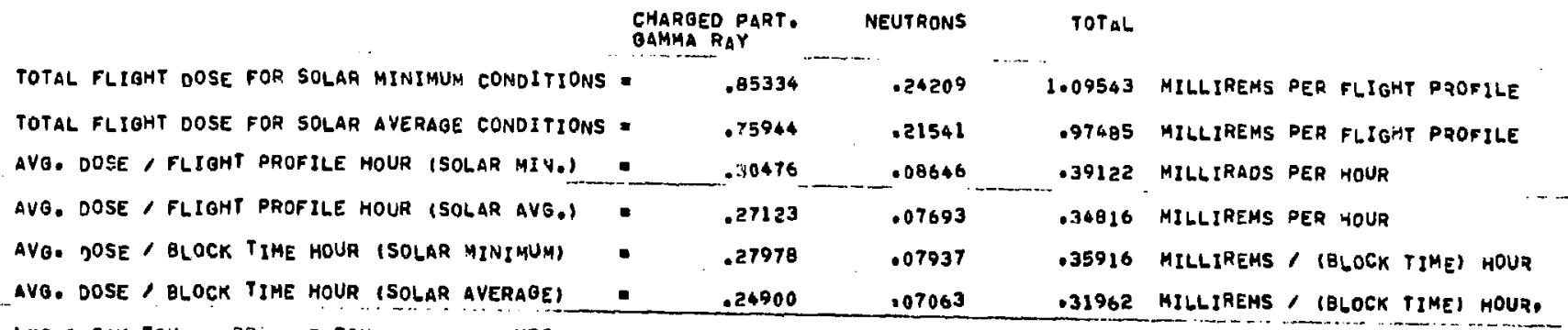

LEO BLOCK TIME - PF: BLE TIME + 0.25 HOURS 
RATE OF CLIMB(FEET/HINI $=20000000$

SCHEDULE BLOCK TIME ON ONE WAY(HRS) *

10.0

CRUISINS ALTITUDE(FEET): 36000 RATE OF DESCENT(FEET/MIV)= 2000.000

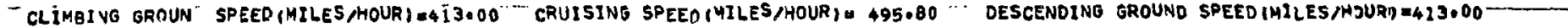

FLIGHT ALTITUDE ANO OISTANCE PROFILE IN 1110 HOUR INCREMENTS.

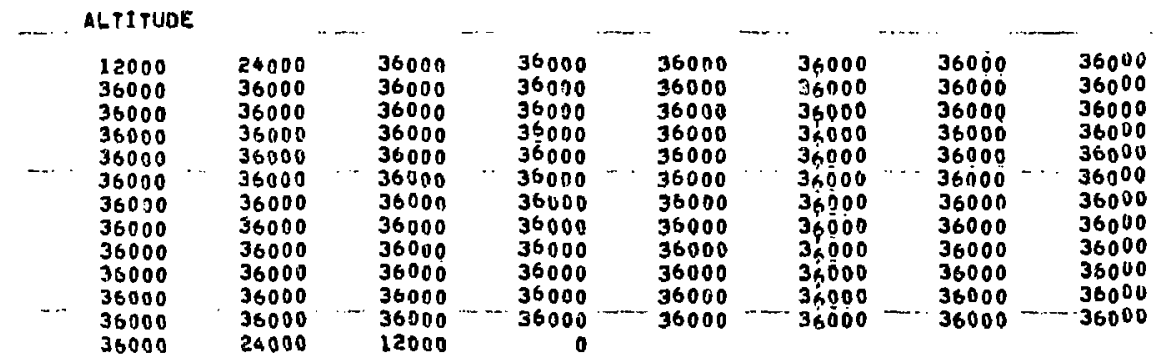

DISTANCE

$\begin{array}{ll}41.30 & 41.30 \\ 49.58 & 49.58 \\ 49.58 & 49.58 \\ 49.58 & 49.58 \\ 49.58 & 49.58 \\ 49.58 & 49.58 \\ 49.58 & 49.58 \\ 49.58 & 49.58 \\ 47.58 & 49.58 \\ 49.58 & 49.58 \\ 49.58 & 49.58 \\ 49.58 & 49.58 \\ 49.58 & 41.30\end{array}$

$\begin{array}{ll}41.3 n & 49.58 \\ 49.58 & 49.58 \\ 49.58 & 49.58 \\ 49.58 & 49.58 \\ 49.58 & 49.58 \\ 49.58 & 49.58 \\ 49.58 & 49.58 \\ 49.58 & 49.58 \\ 49.58 & 49.58 \\ 49.58 & 49.58 \\ 49.58 & 49.58 \\ 49.58 & 49.58 \\ 41.30 & 41.30\end{array}$

$\begin{array}{rrrr}49.58 & 49.58 & 49.58 & 49.58 \\ 49.58 & 49.58 & 49.58 & 49.58 \\ 49.58 & 49.58 & 49.58 & 49.58 \\ 49.58 & 49.58 & 49.58 & 49.58 \\ 49.58 & 49.58 & 49.58 & 49.58 \\ 49.58 & 49.58 & 49.58 & 49.58 \\ 49.58 & 49.58 & 49.58 & 49.58 \\ 49.58 & 49.58 & 49.58 & 49.58 \\ 49.58 & 49.58 & 49.58 & 49.58 \\ 49.58 & 49.58 & 49.58 & 49.58 \\ 49.58 & 49.58 & 49.58 & 49.58 \\ 49.58 & 49.58 & 49.58 & 49.58\end{array}$

TOTAL INPUT PROFILE DISTANCE - 4908.32 STATUTE hIf́Es

FLIGHT PROFILE TIHE

- 10.00 houRs IN TH́E AIR 


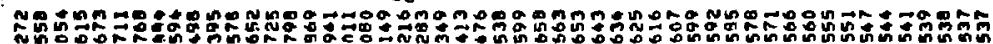
政:

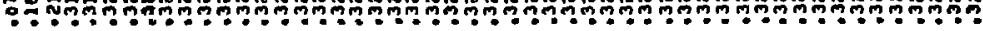

密带

.

Mำ NN

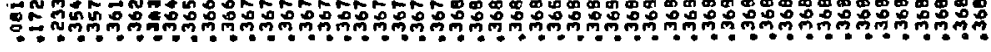

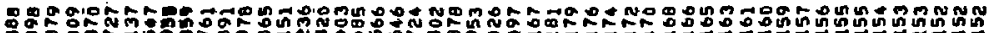

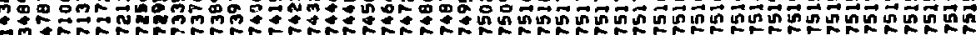

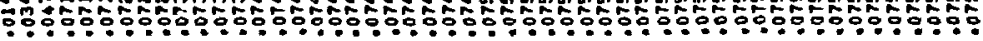
1

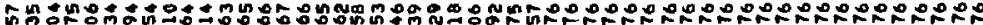

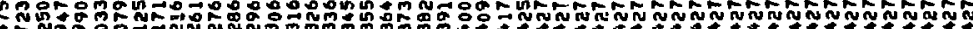

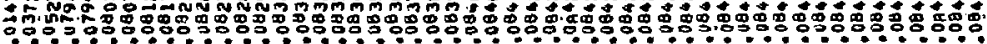

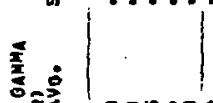<smiles>C1CCC2(CC1)CCCC2</smiles><smiles>IC1CCCC1I</smiles><smiles>C1CCCCC1</smiles><smiles>C1CCCCC1</smiles><smiles></smiles>

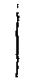

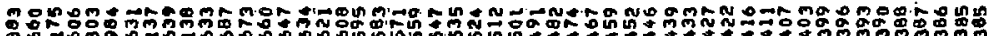

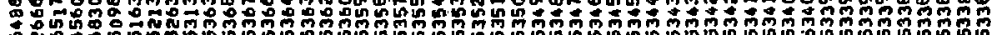
:

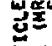
날 1
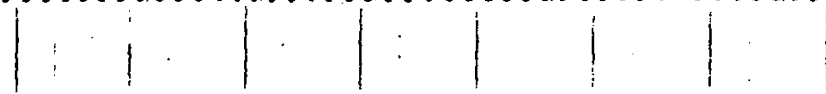

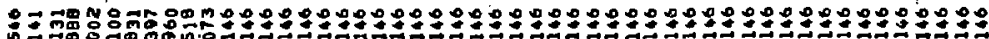

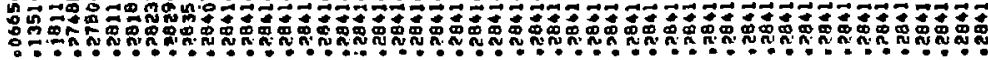

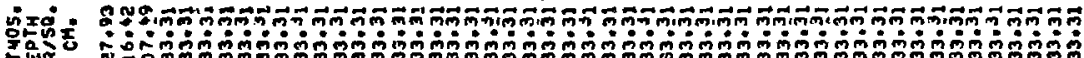
Ш

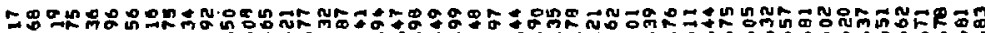

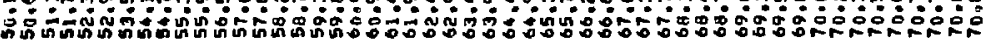

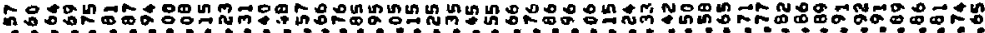

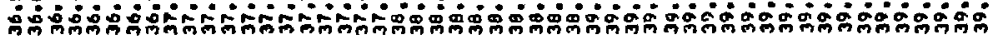

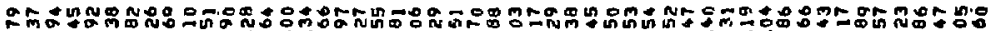

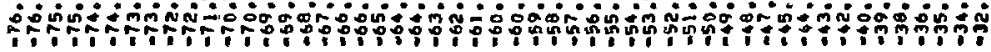

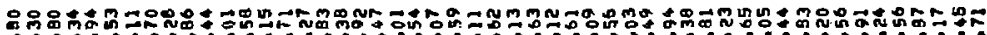

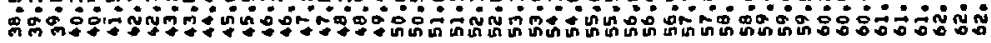




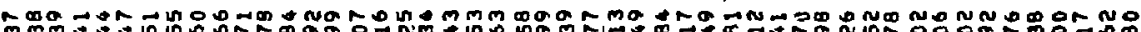
m m

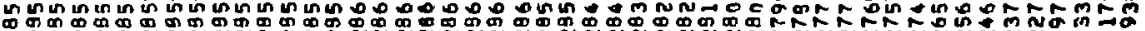

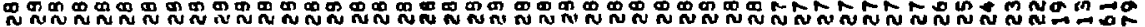

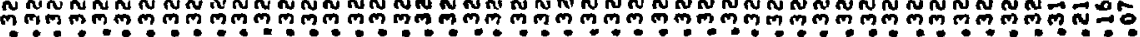

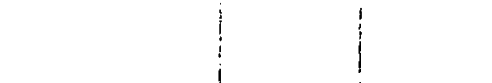

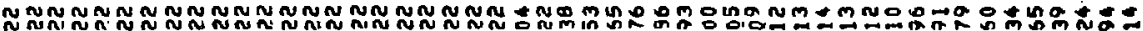
\$

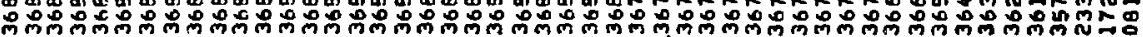

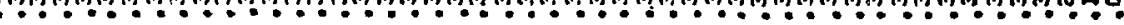

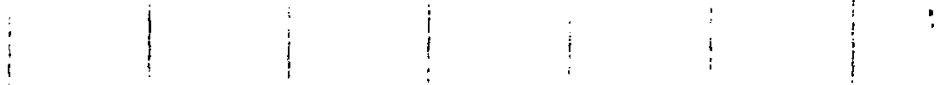

N N $m$ N

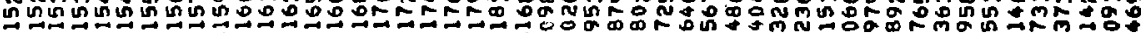

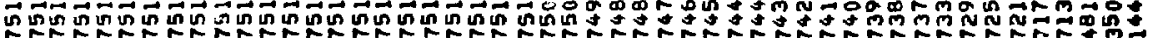

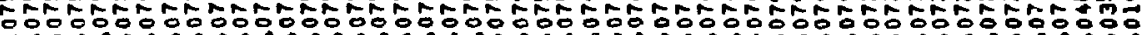
.
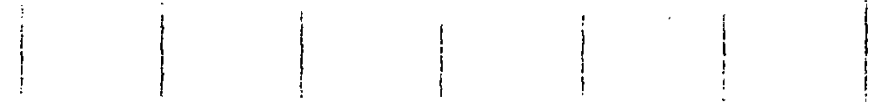

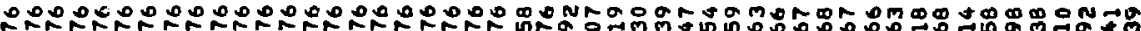

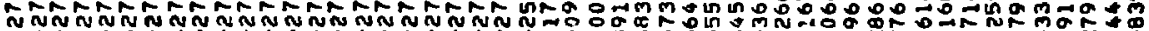

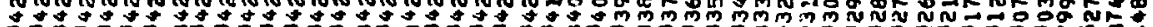
\$

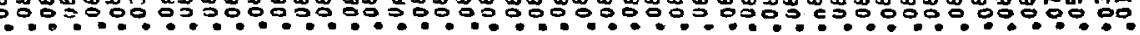
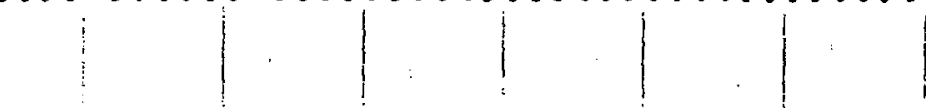

"月

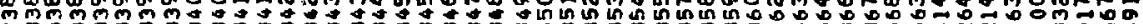

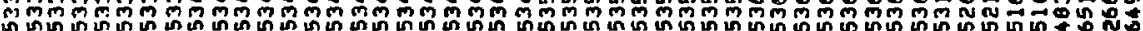

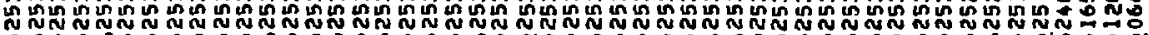

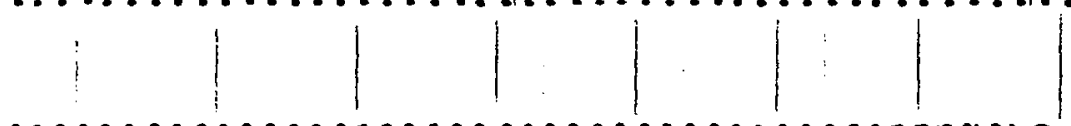

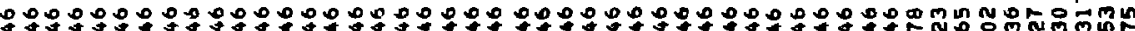

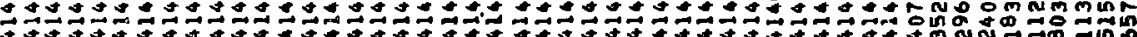

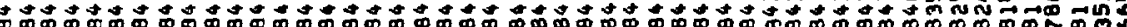

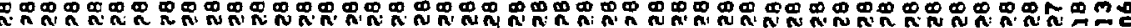

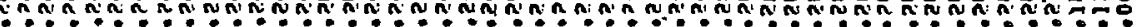

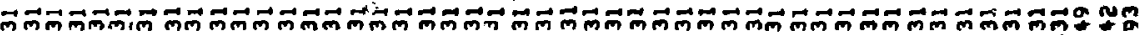

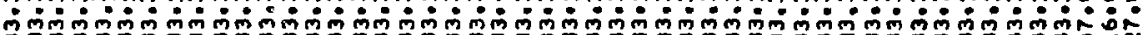

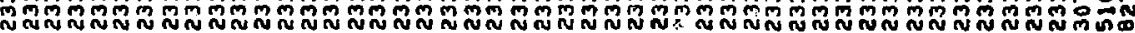

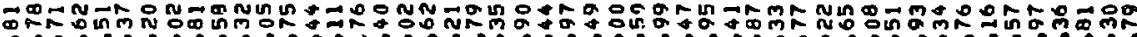
: 을

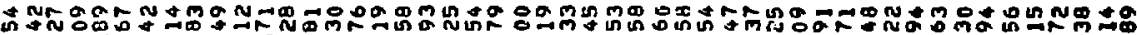

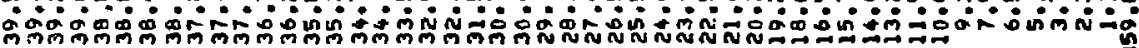

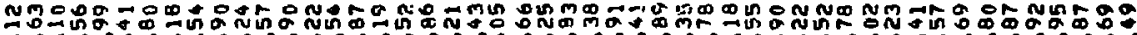
$\rightarrow 0$ :

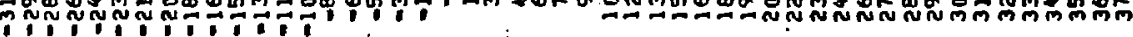

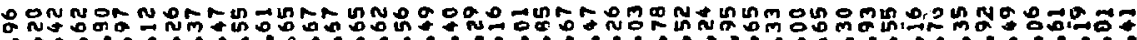

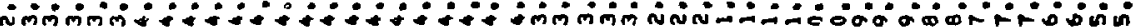

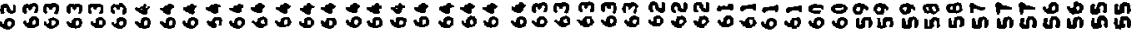




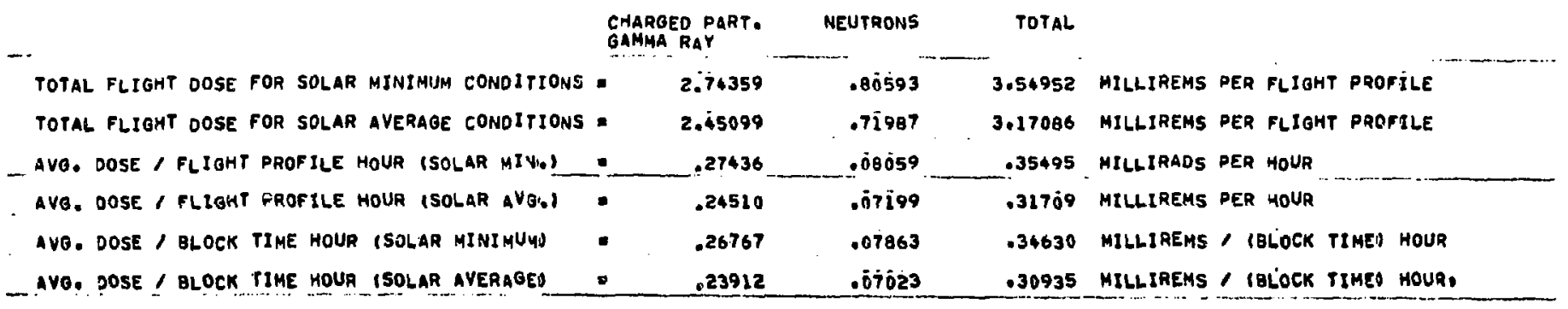

. LEO BLOCK TIME - PROFILE TIME - O.ZS HOURS 
CRUISINO ALTITUDE(FEETIE 36000 RATE OF DESCENTIFEETIMI\$IE 2000.000

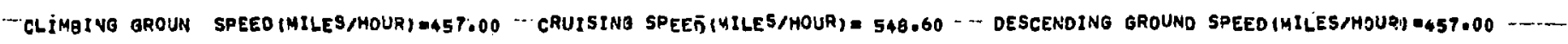

ALTITUDE

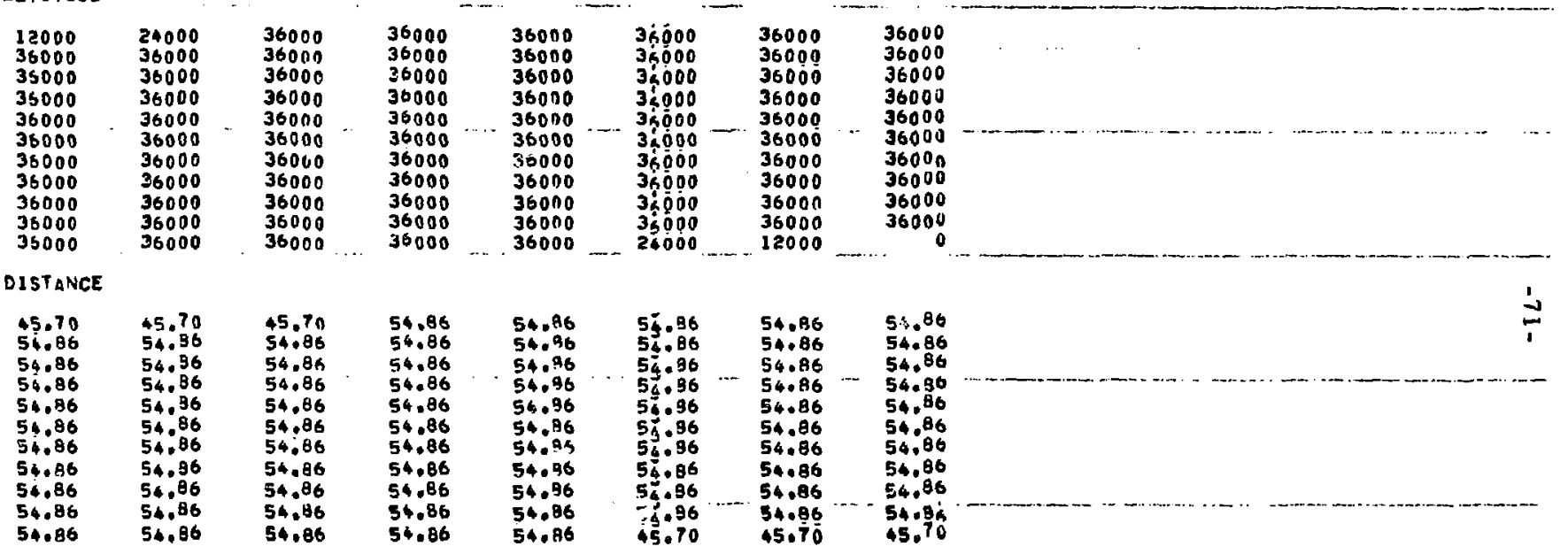

TOTAL INPUT PROFILE DISTANCE.

FLIOHT PROFILE TIHE. 


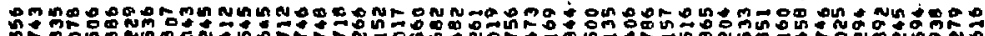

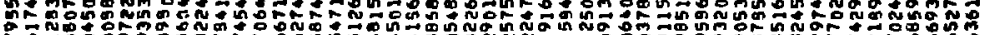
:
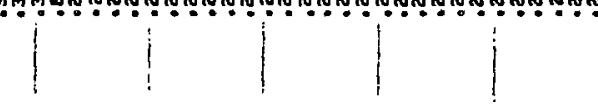

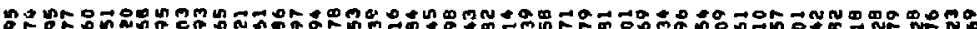

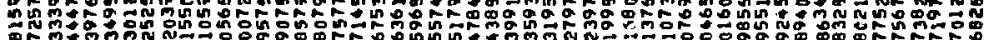

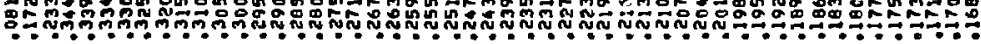<smiles></smiles>
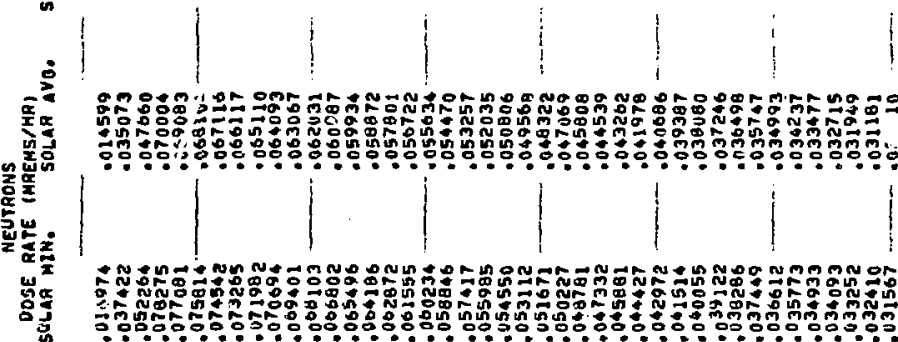

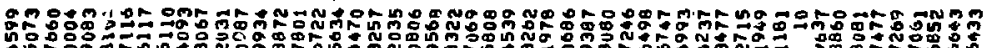

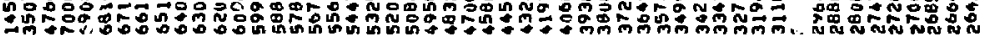

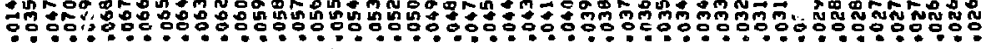
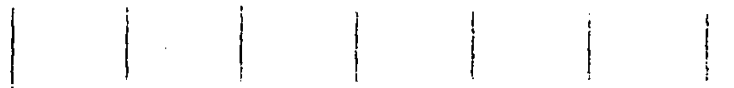

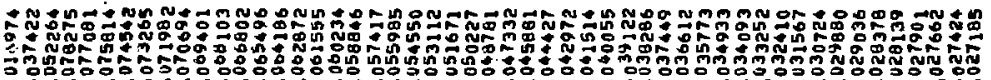
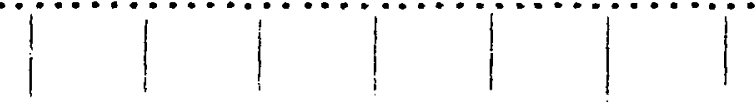

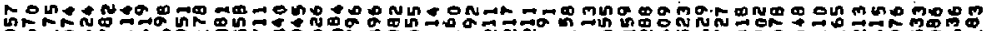

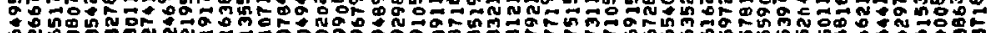

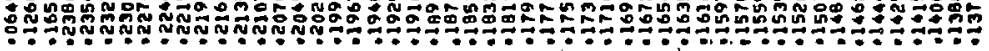
幽要

$\vec{x} \geq$
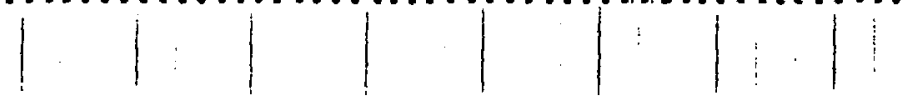

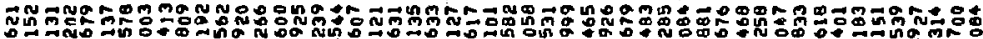

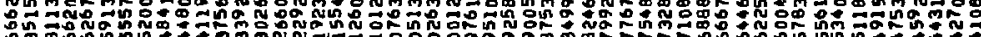

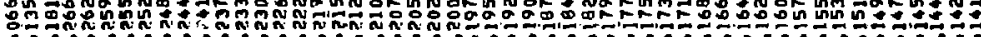

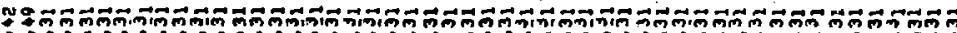

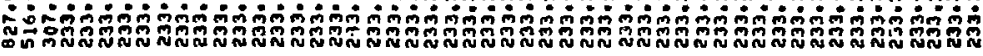

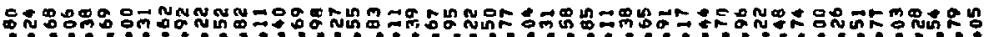
ติง

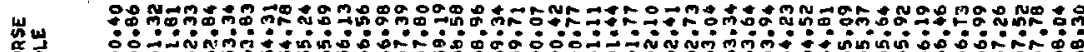

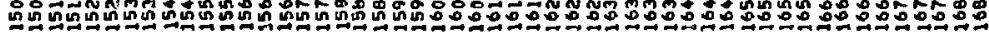

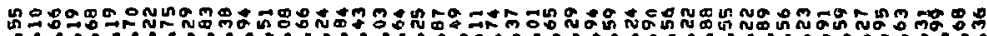

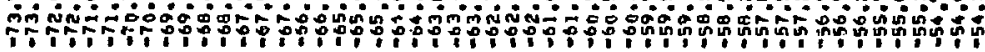

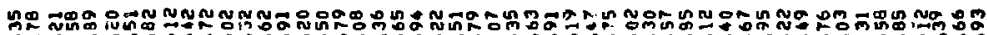
: 


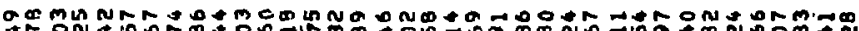

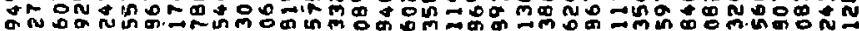

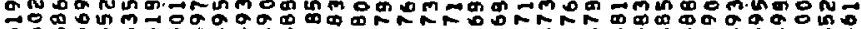
Oo

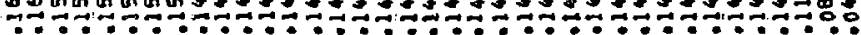
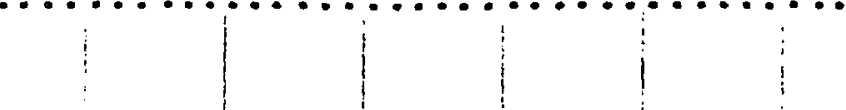

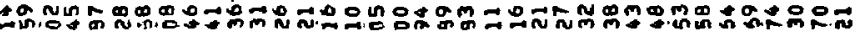

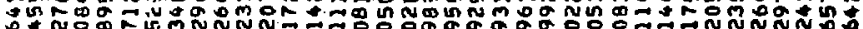

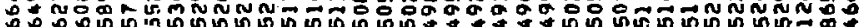

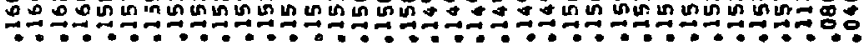
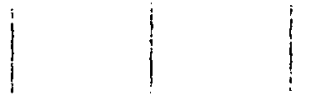

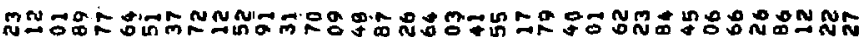

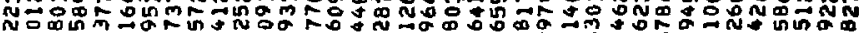

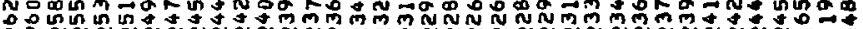

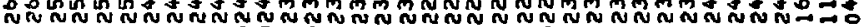

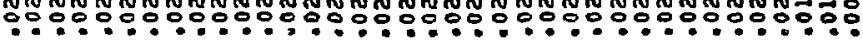
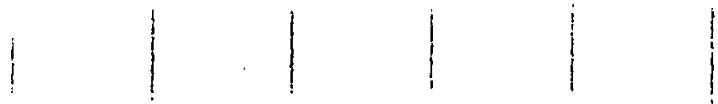

OR

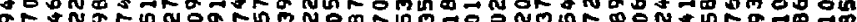

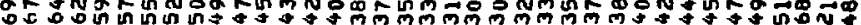

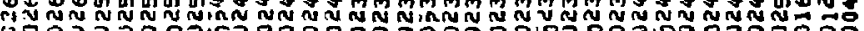

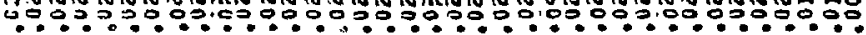

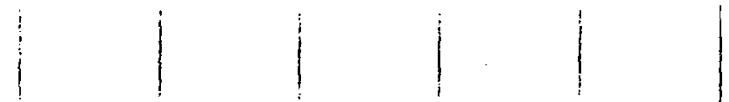

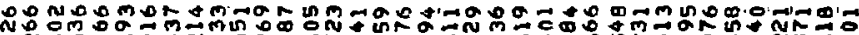

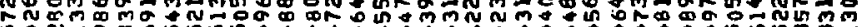

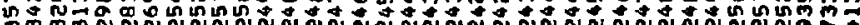
再m N

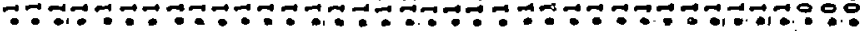
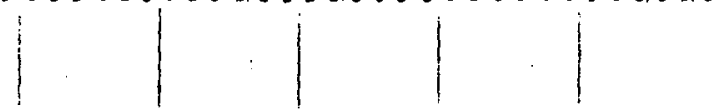

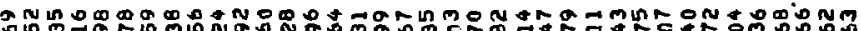

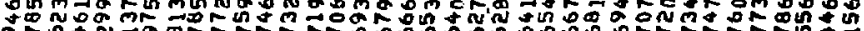
ำ

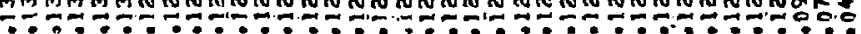

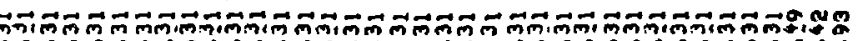
ம்

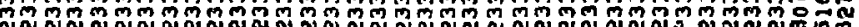

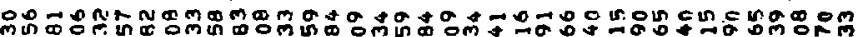

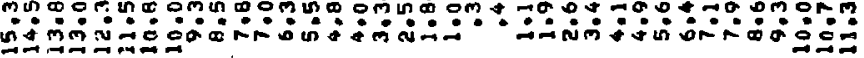

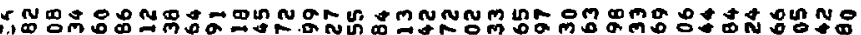
$\because \infty$ ํ.

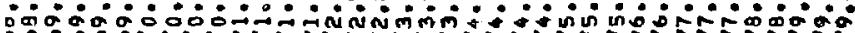

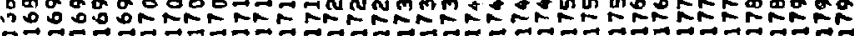

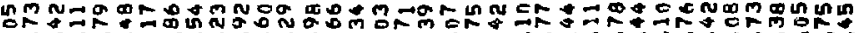
- mininis.

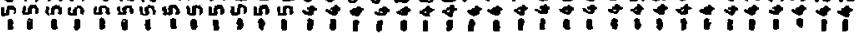

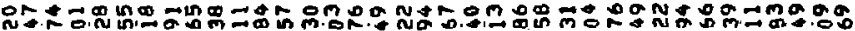

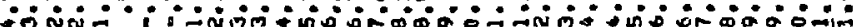
- in 


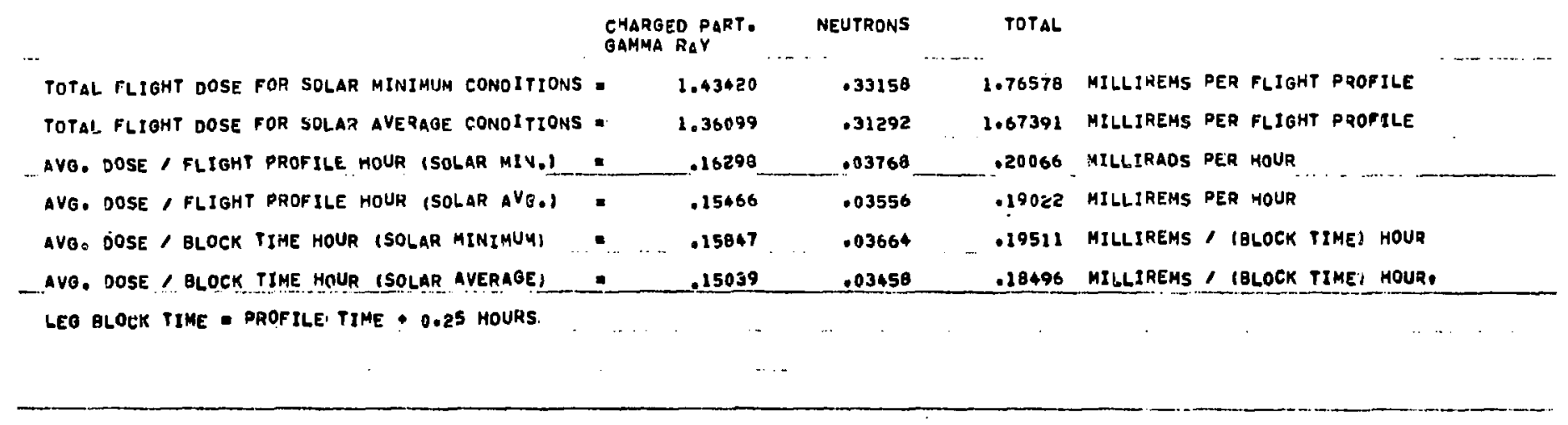

